\title{
THE EFFECTS OF ATOMIC OXYGEN ON PATCH ANTENNA PERFORMANCE AND LIFETIME
}

\author{
A Thesis \\ presented to
}

the Faculty of California Polytechnic State University,

San Luis Obispo

\author{
In Partial Fulfillment \\ of the Requirements for the Degree \\ Master of Science in Aerospace Engineering
}

by

Max J. Barta

July 2019 
(C) 2019

Max Jefferson Barta

ALL RIGHTS RESERVED 
COMMITTEE MEMBERSHIP

TITLE: The Effects of Atomic Oxygen on Patch

Antenna Performance and Lifetime

AUTHOR: Max Jefferson Barta

DATE SUBMITTED: July 2019

COMMITTEE CHAIR: Kira Jorgensen Abercromby, Ph.D.

Associate Professor of Aerospace Engineering

COMMITTEE MEMBER: Amelia Greig, Ph.D.

Assistant Professor of Aerospace Engineering

COMMITTEE MEMBER: Pauline Faure, Ph.D.

Assistant Professor of Aerospace Engineering

COMMITTEE MEMBER: $\quad$ Max Jay Glicklin, M.S.

Sr. Research Scientist at Lockheed Martin 


\begin{abstract}
The Effects of Atomic Oxygen on Patch Antenna Performance and Lifetime Max Jefferson Barta
\end{abstract}

The space environment is a volatile and challenging place for satellites to survive in. For Low Earth Orbiting (LEO) satellites, atomic oxygen (AO) is a constant corrosive effect that degrades the outer surface of satellites over long durations. Atomic oxygen exists in the atmosphere between 180 and $675 \mathrm{~km}$ and has a relatively high energy at $4.5 \mathrm{eV}$, which allows AO to break molecular bonds in materials on the surfaces of spacecraft. As the number and complexity of CubeSat missions increase, there is an increased risk that $\mathrm{AO}$ degradation on commercial off the shelf parts (COTS), such as antenna, could degrade the satellite's ability to communicate with ground systems. This thesis looks at how AO erosion affects the performance of patch antennas for CubeSat applications. Patch antennas are small, cheap, low-profile antennas that can be used on CubeSats to communicate with the ground or other satellites. Patch antennas are semi-directional, providing higher gain and higher available frequencies than omnidirectional antennas. An AO chamber in the California Polytechnic State University San Luis Obispo's (Cal Poly) Spacecraft Environments Testing Lab was used to expose the patch antennas for 24-hour and 48-hour tests. The 24-hour exposure saw an average AO fluence of $8.757 \pm 0.807 \cdot 10_{20}$ atoms $/ \mathrm{cm} 2$ which corresponds to roughly 3.5 months of on-orbit AO exposure on the Ram side when in a $28.5^{\circ}$ inclined orbit with an altitude of $400 \mathrm{~km}$. The 48-hour exposure saw an average AO fluence of $1.595 \pm 0.076 \cdot 1021$ atoms $/ \mathrm{cm}_{2}$ which corresponds to approximately 6.4 months of on-orbit AO exposure on the Ram side when in a $28.5^{\circ}$ inclined orbit with an 
altitude of $400 \mathrm{~km}$. To test the performance of the patch antenna before and after AO exposure, an anechoic chamber in the Microwave Lab at Cal Poly was used to measure boresight gain and radiation pattern in the E-plane and H-plane. From the testing in the anechoic chamber it was determined that there was no apparent difference in the patch antenna's gain and radiation pattern before and after AO exposure. By using a Fourier Transform Infrared Spectrometer (FTIR) it was discovered that the outer surface of the patch antennas were forming a silicon dioxide layer, which did not affect the performance of the patch antenna. Since silicon dioxide is resistant to AO erosion, it may be beneficial for CubeSats to include silica additives to their exposed antenna surfaces to prevent erosion. 


\section{ACKNOWLEDGMENTS}

Thank you to Dr. Amelia Greig, Dr. Pauline Faure, and Max Glicklin for being part of my committee and allowing me to ask them endless questions about every aspect of my thesis.

Thank you to Dr. Dean Arakaki for allowing me to use the anechoic chamber and helping me with hours and hours of antenna testing.

Thank you to Dr. Trevor Harding and the MATE department for allowing me to use their equipment and helping me run the equipment as well as helping me understand how to interpret IR spectroscopy.

Thank you to Dr. Jordi Puig-Suari for helping me come up with the idea for this thesis.

Thank you to my friends and family for motivating me and providing endless support throughout this process.

Thank you to Annie Borland for the motivation, encouragement, and 24/7 love and support. Without it I would never have been able to finish in time.

Thank you to Dr. Kira Abercromby for being my advisor and keeping me on track despite my best efforts to do the opposite. 


\section{TABLE OF CONTENTS}

\section{Page}

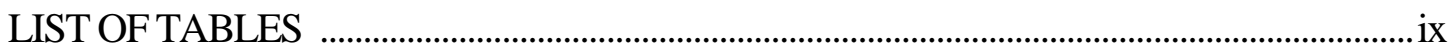

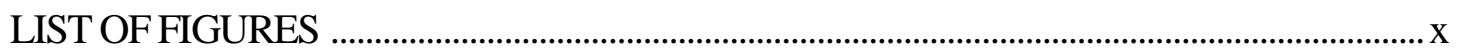

\section{CHAPTER}

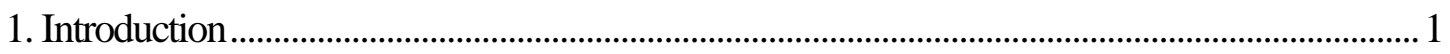

1.1 Space Environments Background ................................................................................... 1

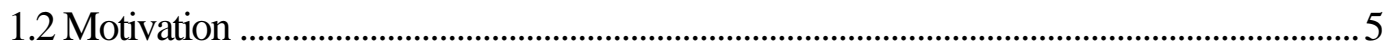

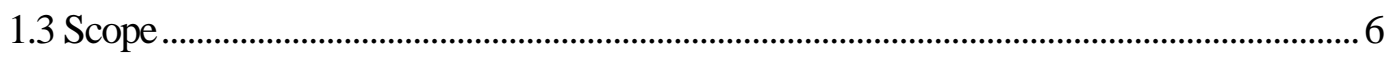

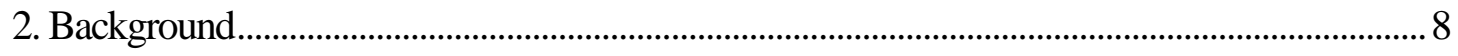

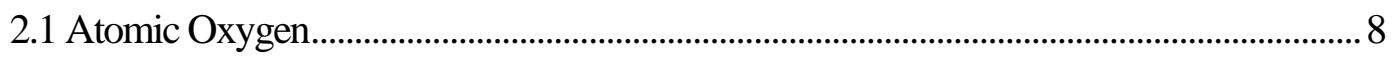

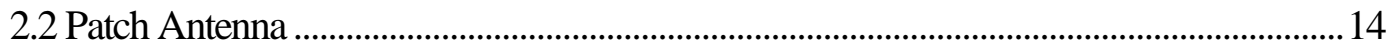

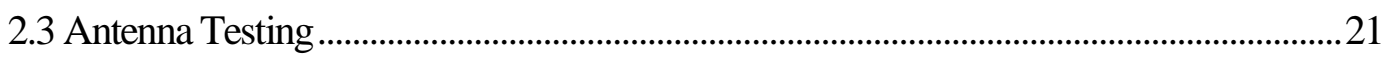

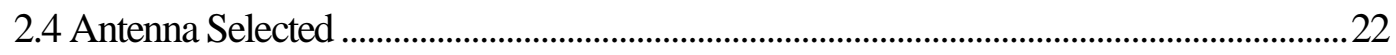

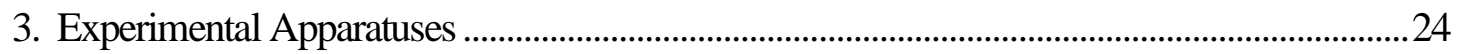

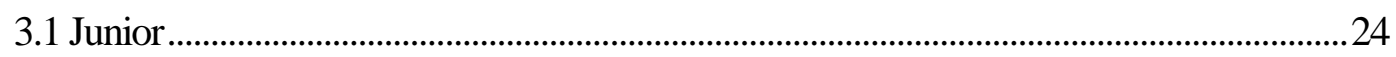

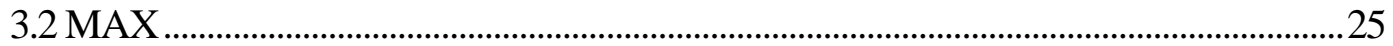

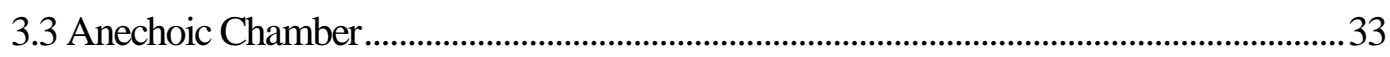

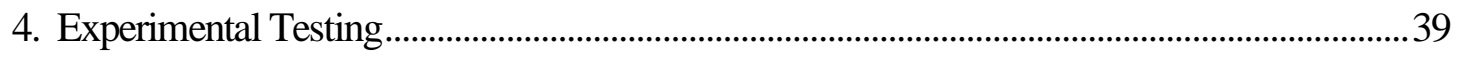

4.1 Antenna Performance Testing ......................................................................................... 39

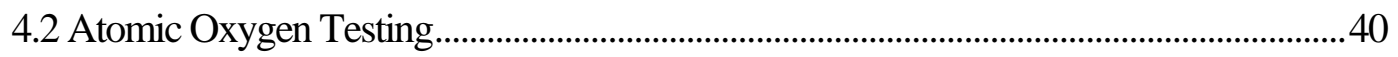

4.3 Scanning Electron Microscope ….................................................................................. 42 
4.4 Fourier Transform Infrared Spectroscopy …................................................................... 43

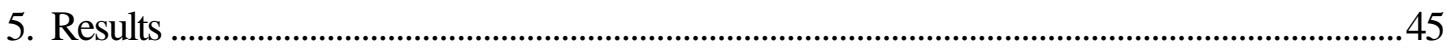

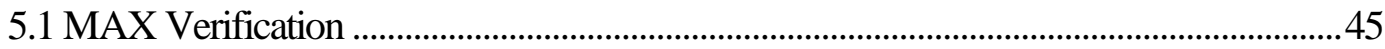

5.2 24-Hour Atomic Oxygen and Antenna Testing....................................................................51

5.3 48-Hour Atomic Oxygen and Antenna Testing....................................................................56

5.4 Scanning Electron Microscope and FTIR Spectroscopy....................................................69

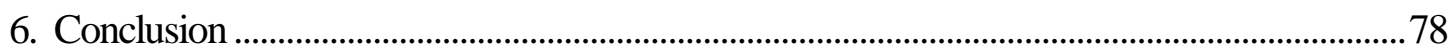

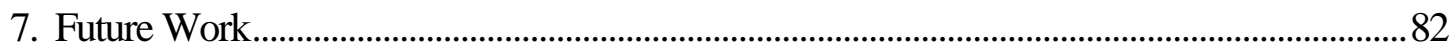

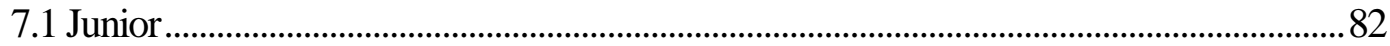

7.2 MAX

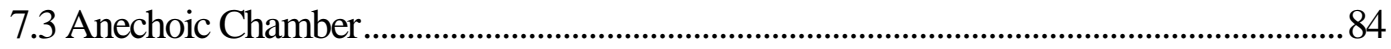

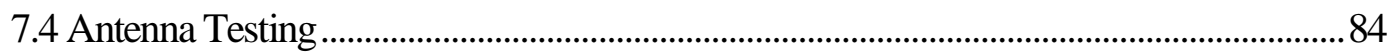

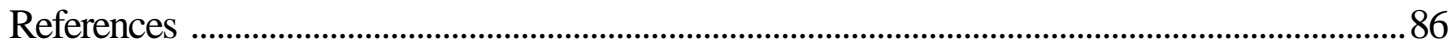

APPENDICES

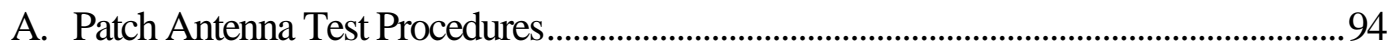

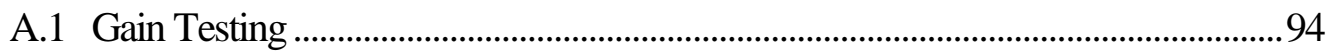

A.2 Radiation Pattern Testing............................................................................................ 96

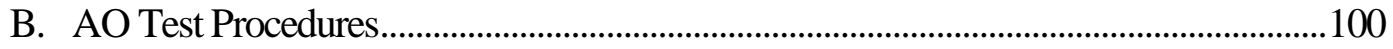

B.1 Vacuum Operating Procedures ......................................................................100

B.2 MAX Operating Procedures..................................................................................... 100

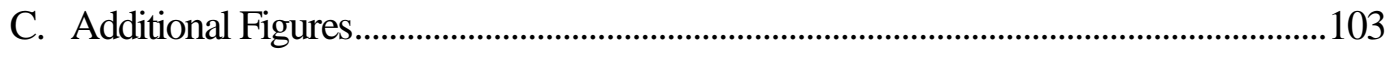

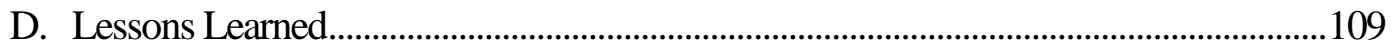




\section{LIST OF TABLES}

Table

2.1 2009-2012 CubeSat Missions Using Patch Antennas [34]

5.1 24-Hour AO Test to Measure Varying Flux and Fluence for Each Sample

Position

5.2 Comparison of the Witness Samples Flux and Fluence for Each AO Test

Conducted

5.3 24-Hour Antenna Mass Loss Values and Flux and Fluence for Each Test....

5.4 48-Hour Antenna Mass Loss and Flux and Fluence for Each Test

5.5 Paired Samples T-Test for 48-Hour Exposed Patch Antenna Gain Over Various

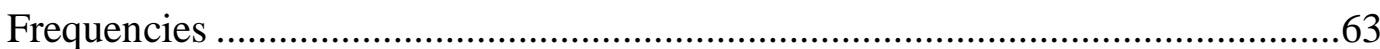

5.6 Peaks of IR Spectra and the Corresponding Chemical Bonds .74 


\section{LIST OF FIGURES}

Figure

2.1 NRLMSISE-00 Atmosphere Model Showing Gas Particle Density Relative to Altitude [9]

2.2 Scanning Electron Microscope Image of Atomic Oxygen Erosion on Kapton [11].

2.3 Erosion of Graphite by Atomic Oxygen Showing Chemical Bonding and

Physical Removal Mechanisms [9] .12

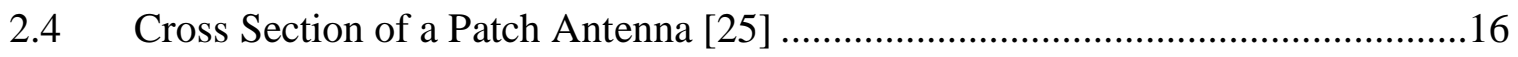

2.5 Dove Satellite with an S-band Patch Antenna [33] ...........................................19

2.6 FXP72 Freedom 2.4 GHz Antenna Manufactured by Taoglass [38]...................23

3.1 Junior Vacuum Chamber Schematic [39]..................................................25

3.2 Simple Diagram of a Capacity Coupled Plasma Created in MAX [40]..............27

3.3 Seren R301MKII Generator Installed in MAX [43] .....................................28

3.4 Seren IPS AT3 Matching Network (Bottom) and MC2 Controller (Top) [43] .....28

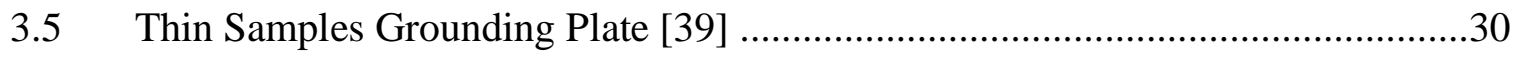

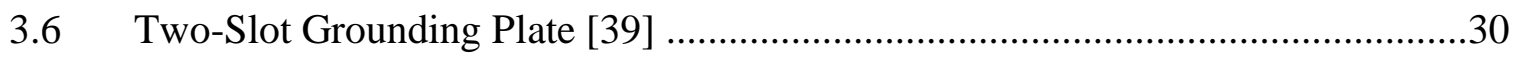

3.7 MAX Creating a Capacity Coupled Plasma to Produce Atomic Oxygen. ............31

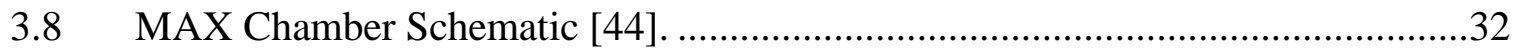

3.9 Cal Poly Microwave Lab Anechoic Chamber Cross Section [46]. .......................34

3.10 Block Diagram of HP8720C Network Analyzer During Operation [47] .............35 


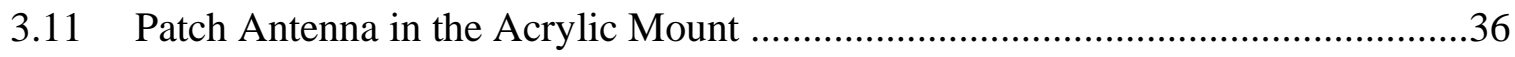

3.12 Patch Antenna in Mount with E-plane and H-plane .............................................37

3.13 Patch Antenna with Coordinate Frame [38] .........................................................38

4.1 Kapton Witness Sample with Edges Sealed by Aluminum Tape ..........................41

5.1 Location of Each Sample in the Trough Along with Witness Sample Location ...46

5.2 Kapton Sample Placement for 24-Hour Test to Measure AO Flux in Different Positions in the Chamber.

5.3 24-Hour AO Exposed Samples that Were Placed in Positions 1 (Left) and 4 (Right) .54

5.4 24-Hour AO Exposed Samples that Were Placed in Positions 2 (Left) and 5 (Right) with an Untested Sample (Middle). .54

5.5 24-Hour AO Exposed Samples that Were Placed in Positions 3 (Left) and 6 (Right). .55

5.6 Antennas 10-15 Change in Gain After 24-Hour AO Exposure .56

5.7 48-Hour AO Exposed Samples that Were Placed in Positions 1 (Left) and 4 (Right)

5.8 48-Hour AO Exposed Samples that Were Placed in Positions 2 (Left) and 5 (Right) with an Untested Sample (Middle).

5.9 48-Hour AO Exposed Samples that Were Placed in Positions 3 (Left) and 6 (Right). .60

5.10 Antennas 16-21 Change in Gain After 48-Hour AO Exposure .61

5.11 Antennas 22-26 Change in Gain After 48-Hour AO Exposure. .62 
5.12 Antennas 16-21 Radiation Pattern in the E-Plane Measured Before (Solid Lines) and After (Dotted Lines) 48 Hours of AO Exposure

5.13 Antennas 16-21 Radiation Pattern in the H-Plane Measured Before (Solid Lines) and After (Dotted Lines) 48 Hours of AO Exposure. .65

5.14 Antennas 22-26 Radiation Pattern in the E-Plane Measured Before (Solid Lines) and After (Dotted Lines) 48 Hours of AO Exposure .65

5.15 Antennas 22-26 Radiation Pattern in the H-Plane Measured Before (Solid Lines) and After (Dotted Lines) 48 Hours of AO Exposure. .66

5.16 Comparison of Patch Antenna Gain with and without a Copper Ground Plane....67

5.17 Comparison of Patch Antenna E-plane with and without a Copper Ground

Plane. .68

5.18 Comparison of Patch Antenna H-plane with and without a Copper Ground

Plane .68

5.19 Patch Antenna Surface (Left) and Patch Antenna (Right) After 48 Hours of

Exposure ........................................................................................ 70

5.20 Control Patch Antenna Surface (Left) and Patch Antenna (right).......................70

5.21 The Effects Charging Has on Images Taken by the SEM................................71

5.22 Attenuated Total Response IR Spectrum of Control Antenna............................72

5.23 Attenuated Total Response IR Spectrum of the Control Antenna (Red), 24-

Hour Sample (Green), and 48-Hour Sample (Blue). .......................................74

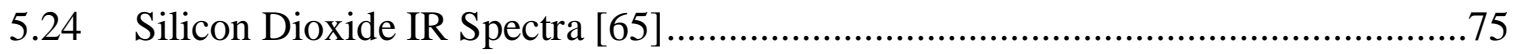

5.25 Silicon Dioxide Thickness vs Observed Color at $0^{\circ}$ Incidence Angle [66]..........76

5.26 Color Gradient Seen on the 48-Hour Patch Antenna Sample..............................76 
A.1 Anechoic Chamber with Reference Frame Centered at the Test Antenna Position

A.2 Flow Chart for Choosing Radiation Pattern Measurement Setting.......................99

C.1 VNA Used in the Anechoic Chamber ....................................................... 103

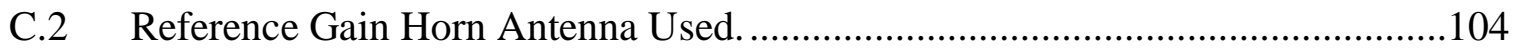

C.3 48-Hour Sample (Left) Compared to a 24-Hour Sample (Right). .......................105

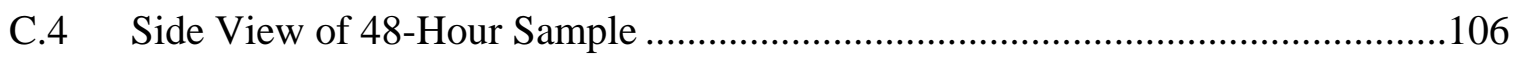

C.5 Scanning Electron Microscope Used for Imaging Samples............................. 107

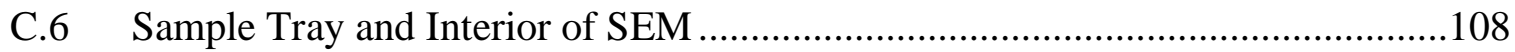




\section{Chapter 1}

\section{INTRODUCTION}

This chapter delves into the various aspects of the spacecraft environment and how they can affect spacecraft. First, an overview of the different parts of the spacecraft environments is examined. Next, the motivation for this thesis and goals of the research are discussed. Finally, the end of the chapter explains the scope of the thesis and its relevance to spacecraft.

\subsection{Space Environments Background}

The space environment is an extremely volatile environment in which spacecraft face conditions that are very different than on the surface of Earth. Satellites in the space environment are exposed to the neutral environment, plasma, radiation, particulate, and intense thermal cycling. To ensure a spacecraft can effectively operate for its mission's required lifetime, each aspect of the space environment must be accounted for in the satellite's design.

To start off the spacecraft must be able to operate in the neutral environment of space. The neutral environment includes Earth's atmosphere which decreases in density at an exponential rate as altitude increases, and by an altitude of $100 \mathrm{~km}$ the density of the atmosphere is only $5.08 \cdot 10_{-7} \mathrm{~kg} / \mathrm{m} 3$. A lack of atmosphere means the material on the spacecraft may outgas - a phenomenon that occurs when trapped particles of gas or water are released by a material as a result of entering a lower pressure. It is desirable for manufacturers to use materials that meet the standards set by ASTM E-595-93 due to this effect [1]. This standard limits the total mass loss (TML) of the material to be less than $1.0 \%$ and the collected volatile condensable mass (CVCM) to be less than $0.10 \%$ [1]. 
Another part of the neutral environment that can disrupt satellites is Atomic Oxygen (AO) [1]. AO is formed from the dissociation of diatomic oxygen molecules by solar radiation to form monatomic oxygen particles. Typically, AO is found between 60 and $800 \mathrm{~km}$ and is the dominant atmospheric constituent from 180 to $675 \mathrm{~km}$. The AO particles erode layers of material in the Ram direction of the satellite. More background on AO will be discussed in a later section.

Plasmas are thought to compose up to $99 \%$ of the mass in the universe [1]. Plasma makes up the interior of stars, solar wind, and parts of the atmosphere of planets - like the ionosphere of Earth - and consists of positively charged ions, electrons, and neutral particles. The particles' interactions with a spacecraft can affect communication, cause differential charging, and cause sputtering on spacecraft surfaces [1]. The plasma in the ionosphere creates the ionosphere critical frequency, which is the minimum frequency that an electromagnetic signal can possess while traveling through the plasma [1]. This limits the lower bound of available frequency that satellites can use to communicate with the ground. Satellite charging varies depending on the solar activity and where the satellite is exposed to sunlight or eclipse. The higher relative velocity of electrons compared to ions in a plasma results in a net current that can lead to high charging differentials and arcing. Arcing onboard the spacecraft can easily destroy solar panels or other sensitive electronics [1]. Another effect of plasma interactions is sputtering, a phenomenon where ions and neutrals impact and remove the surface atoms of materials [1]. Impacted surfaces with thin films can be particularly sensitive if the film has a low surface binding energy. For this to occur, the impacting particle must have an energy higher than the surface binding energy of the target atoms [1]. 
Radiation in space is a serious issue for electronics onboard a spacecraft. While satellites orbiting Earth do have the magnetosphere protecting them; they do not have the protective shield of Earth's thick atmosphere to block radiation [1]. The radiation experienced by a satellite in orbit around Earth consists of four main types - solar wind, planetary radiation belts, galactic cosmic rays (GCR), and solar particle events (SPE) [1]. Electronics, like semi-conductors, are especially sensitive to radiation which can have both temporary and permanent effects. The three primary effects radiation has on these electronics are displacement damage, total ionizing dose, and single-event effects [1]. Displacement damage occurs when the radiation displaces an atom from its lattice structure [1]. Equipment such as solar cells are particularly susceptible to displacement damage and can experience a decrease in photovoltaic efficiency [1]. Total ionization dose is the accumulated effect of ionizing radiation on equipment during the course of the mission. In semiconductors, the ionizing radiation creates holes that result in current leaks that can build up, causing the semiconductor to short [1]. To reduce these effects, electronics that have been made radiation resistant or semi hard, are used to prevent these effects [1]. Single-event effects come from only a small number of radiation particles. When these particles impact devices, they create ionization trails that can result in bit flips or excessive power draw by the affected electronics [2]. These effects can be temporary or permanent depending on the strength and damage of the ionizing radiation the latter of which was seen on Telstar 1 when radiation degraded its transistors [2].

Particulate debris can be one of the most dramatic aspects of the space environment as it consists of micrometeoroids and space debris. Micrometeoroids are defined to be meteors with a mass of less than 1 gram and space debris consists of any man-made space junk or 
objects from past missions. These bits of particulate can be moving at high relative velocities to the satellites they impact, resulting in hypervelocity impacts. Most humanmade orbital debris is located in a low earth orbit, which is where the majority of CubeSats operate [3]. Several recent demonstrations of antisatellite missiles by India and China have significantly increased the amount of manmade debris increasing the probability of impacts on satellites in similar regions [3]. The increasing amount of debris requires satellites that have the capability to perform more orbital maneuvers to avoid impacts [4]. The department of defense is capable of tracking objects as small as $10 \mathrm{~cm}$ in diameter, however many pieces of debris are significantly smaller than the minimum trackable size [4]. These smaller pieces still pose a significant threat to satellites, as an impact of a small piece of debris or micrometeoroid such as a $1 \mathrm{~cm}$ paint fleck can have the same energy as a 249.5 $\mathrm{kg}$ object traveling at $96.5 \mathrm{~km} / \mathrm{h}$ [4]. These impacts can cause structural damage, contamination, and momentum transfer, which can all adversely affect the satellite's mission. To mitigate the risks posed by these threats, the ram side of satellites and sensitive equipment can be fitted with thicker shielding or Whipple shields [1]. For impact velocities less than $3 \mathrm{~km} / \mathrm{s}$ a thicker layer of shielding is more effective at protecting sensitive equipment, as the projectile impacting the satellite is not likely to vaporize [1]. Above 3 $\mathrm{km} / \mathrm{s}$, a Whipple shield is more effective as it allows for mass savings while still vaporizing the projectile upon impact [1].

The lack of an atmosphere also effects the thermal subsystem of the spacecraft, as the main form of heat dissipation must come from radiation. To allow the satellite to effectively operate, a thermal control system is necessary to prevent either over heating or freezing of spacecraft components. Although the extent of each thermal control system is dependent 
on the orbit and mission that the satellite will be operating at, some form of a thermal control system is necessary to handle the temperature fluctuations in space [1]. The spacecraft must be able to withstand extreme thermal differences as the spacecraft is exposed to the sun and Earth's shadow. Onboard electronics such as computers, batteries, solar panels, and sensitive payload equipment have optimal operational temperatures, but must remain within their survival temperature ranges, which are slightly less restrictive [1]. For some missions the thermal environment may require the satellite to have onboard cryogenic cooling systems to keep the payload within operational temperatures [1]. These temperature ranges can vary for different spacecraft components making the problem of temperature control even more difficult.

\subsection{Motivation}

The space environment can cause a plethora of issues for satellites during their time in space. To lessen the risk of satellites failing on orbit, tests are performed prior to launch to discover and mitigate any potential issues that may arise over the course of the mission. CubeSats, like traditional satellites, must undergo a series of tests to prove the system is sound and satisfies the launcher requirements. For antennas onboard both traditional satellites and CubeSats, functional testing has been conducted by the satellite manufacturer with respect to thermal control, contamination, and pointing. As technology advances, the number and complexity of CubeSat missions are increasing [5]. Recently, there has been increased interest and development in CubeSat constellations and swarms which has caused an increased demand for low cost communication systems. This increase in CubeSat constellation missions could mean long-term exposure to $\mathrm{AO}$ and its associated erosion effects. These effects have not yet been studied on COTS parts for CubeSats since testing 
can be expensive, however the corrosive effects could cause a decrease in antenna performance and should therefore be further explored. For this paper an FXP72 Freedom 2.4 GHz Series Ground Coupled Antenna was exposed to AO to simulate several months of on-orbit degradation. The FXP72 was chosen due to its low profile, light weight, low cost, and commercial off the shelf availability, which are all beneficial for CubeSat missions. The research will show whether AO degradation has an effect on the antenna's performance or lifetime, and to find a way to shield the antenna from this degradation without creating a decrease in the antenna performance in case it is proven that $\mathrm{AO}$ exposure degrades antenna performance.

\subsection{Scope}

This thesis will be focusing on a COTS patch antenna due to its low cost, low risk, and low profile. Because of these factors, the antenna is well suited for CubeSat missions that may require a low-profile antenna to conform to the volumetric requirements of a CubeSat and not run the additional risk of having a deployable antenna. The specific patch antenna chosen for testing has not flown on a mission, however other CubesSat missions such as Dove-1 and Dove-2 have used S-band patch antennas for data downlink [6]. CubeSats were the focus of the thesis as traditional longer lasting satellites have coated their antennas in germanium to act as a protective coating from AO. For CubeSats this is impractical as germanium can be very expensive, which is not desirable for CubeSats. For previous CubeSat missions, AO exposure has not been a major factor in the design and selection of a CubeSats communication system as the satellites would usually stop working before AO could pose a serious problem to any of its onboard systems. As CubeSats develop as a platform, their capabilities are increasing. New CubeSats are being designed with onboard 
active control systems like reaction wheels and micro propulsion that will allow the satellite to remain in orbit for longer. The reaction wheels can allow a CubeSat to orientate itself so that it minimizes its coefficient of drag thereby preserving its orbital altitude. Micro propulsion allows for the CubeSat to perform orbit raising maneuvers to extended the satellites time on orbit. As the number of CubeSat missions increase, AO may become a relevant factor in the design of CubeSats. For these reasons, it was decided that a patch antenna would be tested in an anechoic chamber both before and after exposure to AO to determine whether there was a significant drop in antenna performance. After the performance tests, if there was a change in performance, it was also necessary to determine the material forming on the surface of the antennas to determine whether it was the erosion that was affecting the antenna performance or an oxide layer that formed on the surface that was affecting the antenna performance. 


\section{Chapter 2}

\section{BACKGROUND}

This chapter focuses on the background of atomic oxygen, patch antennas, antenna testing, and the selected patch antenna. First in the chapter is an in-depth background on atomic oxygen followed by a look at patch antennas. Next, is a look at antenna testing that is typically done before an antenna is used on a satellite. The last section in the chapter discusses the selected patch antenna.

\subsection{Atomic Oxygen}

Atomic oxygen is a single atom of oxygen formed by the dissociation of diatomic oxygen. AO is created when photons from the Sun's ultraviolet radiation, specifically wavelengths less than $243 \mathrm{~nm}$, photodisassociates diatomic oxygen [7][8]. On its own, diatomic oxygen is a stable molecule, and in order to separate into AO the molecule requires a dissociation energy of $5.12 \mathrm{eV}$ [9]. In this process the $5.12 \mathrm{eV}$ diatomic chemical bond between oxygen atoms is broken by high energy photons creating two separate atoms [7]. Normally these atoms would recombine with other molecules of ozone in the atmosphere, but the mean free path between these molecules is on the order of 108 meters, making competitive recombination unlikely to occur [8]. AO is found in Earth's atmosphere from $60 \mathrm{~km}$ to $800 \mathrm{~km}$ as the density of the atmosphere at these altitudes is low

enough that recombination of $\mathrm{AO}$ and diatomic oxygen or nitrogen is improbable. Above $800 \mathrm{~km}$ the average molecular weight decreases, so AO is replaced by lighter molecules as the dominant species. From $175 \mathrm{~km}$ to $600 \mathrm{~km} \mathrm{AO}$ is the dominant species in the atmosphere as is represented by figure 2.1. The figure was created using the Naval 
Research Laboratory Mass Spectrometer Incoherent Scatter Model (NRLMSISE) from the year 2000 .

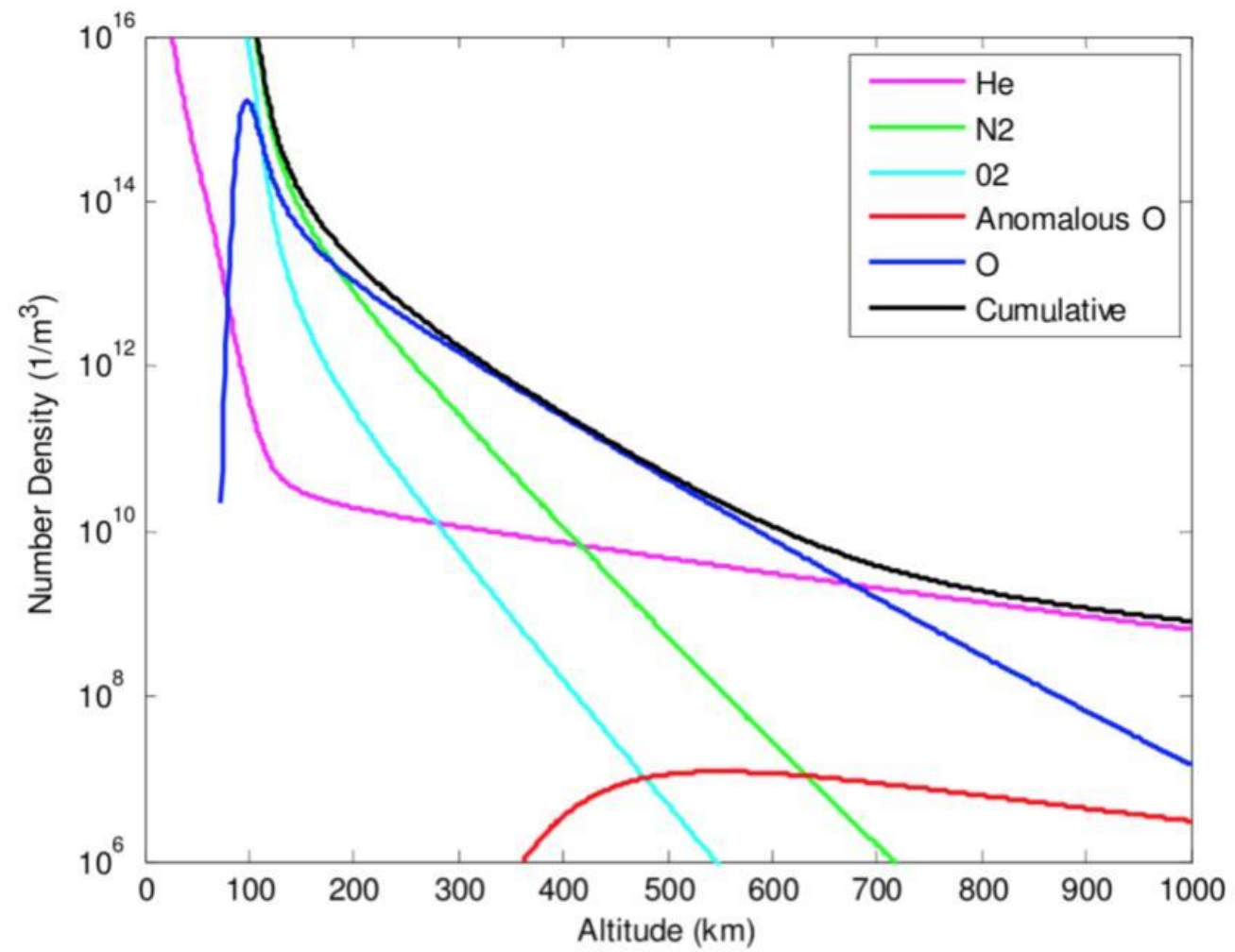

\section{Figure 2.1: NRLMSISE-00 Atmosphere Model Showing Gas Particle Density Relative to Altitude [9].}

Some variations in the altitudes previously discussed can occur as the Sun's solar cycle progresses due to increased or decreased amounts of solar radiation. The three types of satellite orbits that operate at this altitude are Low Earth Orbits (LEO), Polar Earth Orbits (PEO), and Molniya Orbits, however, due to the extremely eccentric nature of Molniya Orbits, AO is typically not a concern as the satellite spends relatively small amounts of time at altitudes with AO. For LEO and PEO, atomic oxygen is a prominent concern as satellites in these orbits can spend their entire lives exposed to AO. 
The space community has known about the existence of AO for many years however the damage caused by its erosion was not seen until the Space Shuttle began flying lower LEO missions [8]. The first indication of AO came from glow on the ram side of the shuttle that was observed by the astronauts onboard [10]. This glow was the result of de-excited molecules and atoms leaving the surface of the shuttle after being removed by AO. The next indication came from the change in reflectance of polymers like Kapton as the AO would alter the texture of the surface of the polymers through sputtering and lower the reflectance of the material as the surface became rougher [7]. After these discoveries, Space Shuttle missions began testing the erosive effects of $\mathrm{AO}$ on other polymer spacecraft materials.

For satellites in LEO or PEO, the highly reactive nature of AO causes oxidative erosion of many materials on the spacecraft. The effects of this erosion are significantly increased on the ram side of the spacecraft due to the reactive particles also possessing high impact energy, which can result in collision energies of $4.5-5 \mathrm{eV}$ between the spacecraft and the AO particles. AO reacts differently with different materials; the five basic mechanisms are abstraction, addition, elimination, insertion, and replacement [10]. Abstraction occurs when AO removes molecules or atoms like hydrogen from a material. Addition is when the oxygen particle attaches or adds itself to another molecule. Elimination occurs when the oxygen atom attaches to a molecule resulting in a vibrationally excited molecule which then eliminates or expels a hydrogen atom. Insertion is when a particle of AO forces itself between two atoms of a molecule. Finally, replacement occurs when the oxygen atom attaches to part of a molecule and the remaining portion of the molecule is expelled, usually as a radical. In polymers, these mechanisms lead to the volatile oxidation of materials that 
previously were part of the material surface. These mechanisms result in mass loss or gain and can lead to changes in surface morphology, thermal, optical, mechanical, or structural properties of materials [8]. When $\mathrm{AO}$ erodes a polymer material on-orbit by abstraction or volatile oxidation, the surface texture becomes rougher than it originally was. The kinetic energy of the AO moving at orbital velocities creates microscopic cones in the surface of the material. On the surface of a sample exposed to $\mathrm{AO}$ on-orbit, the erosion at one location is independent of any other location and the molecules of $\mathrm{AO}$ can impact at any random location [8]. These two characteristics combine to cause the pits and cones seen on the surface of exposed samples [8]. Figure 2.2 shows the pits and cones formed on a sample exposed to $\mathrm{AO}$ as well as a bit of metallic contaminant which forms the cone in the center.

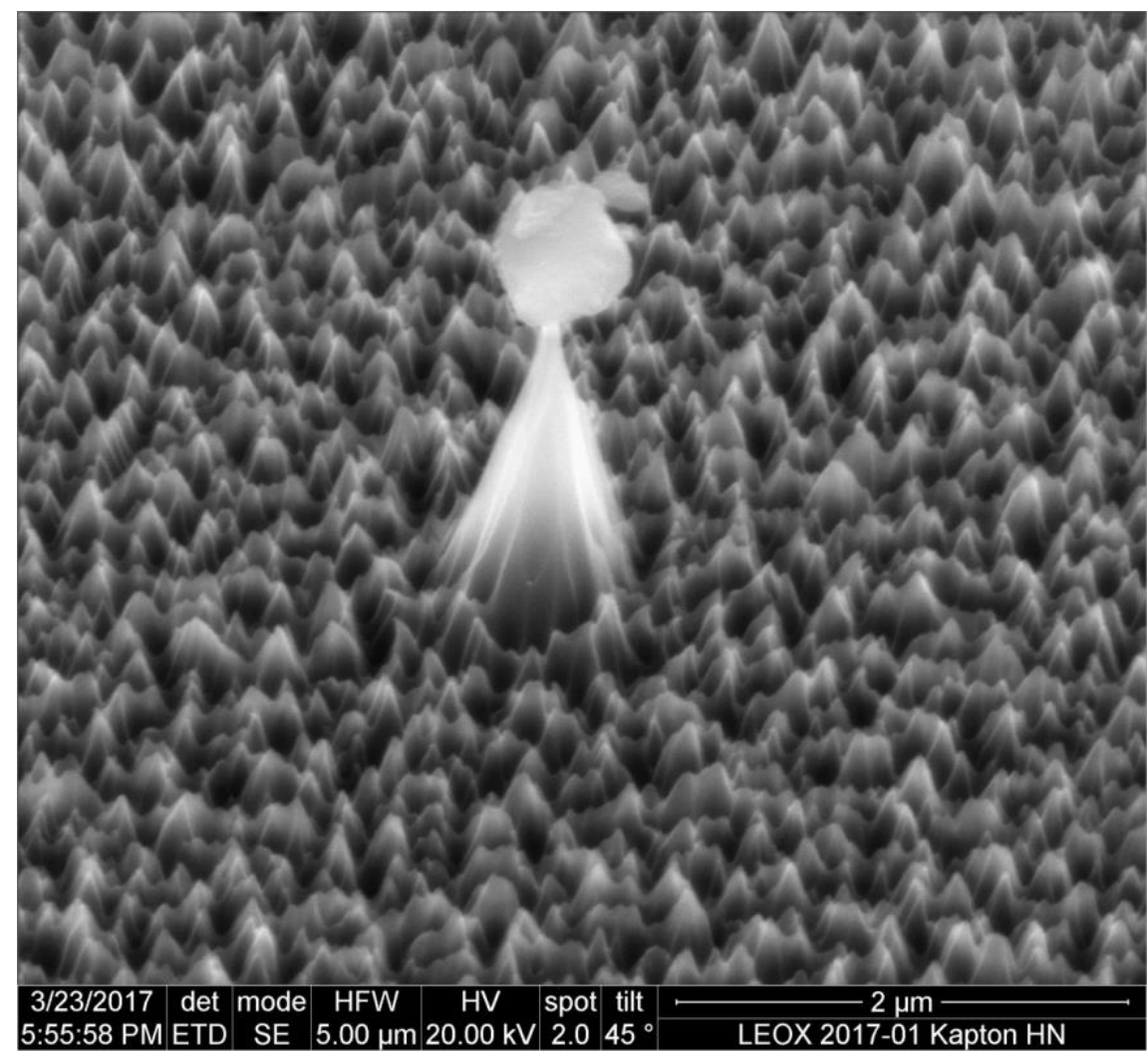

Figure 2.2: Scanning Electron Microscope Image of Atomic Oxygen Erosion on Kapton [11]. 
Additionally, the change in the surface texture of the materials can negatively affect the optical properties of the material. The increased roughness decreases most polymer's specular transmittance, while increasing the polymer's diffuse reflectance which can have a negative effect on the thermal and optical properties of the satellite [12]. For materials such as metals, the interaction with AO is slightly different. Instead of continuously eroding the material, the $\mathrm{AO}$ erodes an initial outer layer, but then the oxygen atoms bind with the metal creating a protective metal oxide layer on the surface of the material. Although this still affects the reflectivity and thermal properties of the material, the process of erosion is severely hampered by the metal oxide. The steps of erosion from a graphite sample can be seen in figure 2.3.

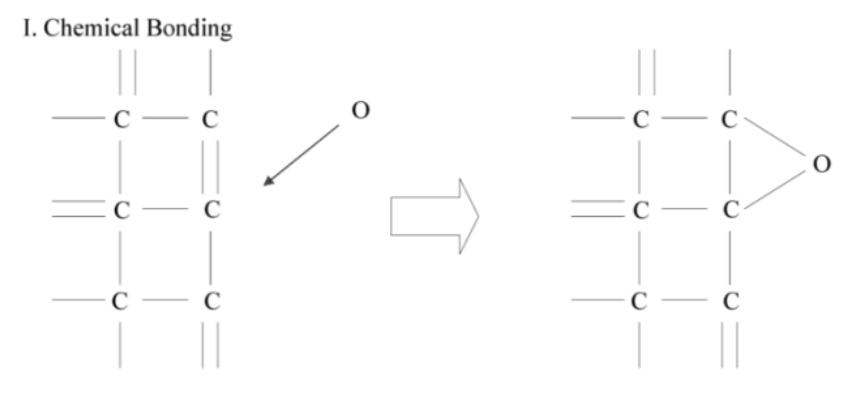

II. Physical Removal
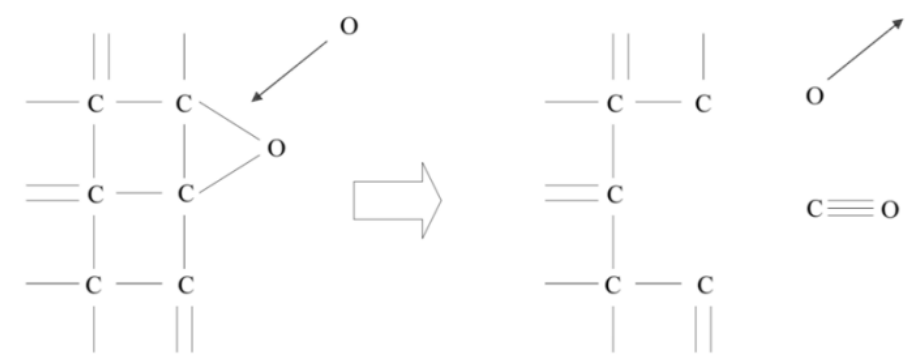

\section{Figure 2.3: Erosion of Graphite by Atomic Oxygen Showing Chemical Bonding and Physical Removal Mechanisms [9].}

In the past, many studies have been conducted on the effects of $\mathrm{AO}$ on different types of materials. Tests were carried out on the Space Shuttle and the International Space Station to observe how exposure to $\mathrm{AO}$ changed the properties of different materials [13]. After 
the shuttle missions, AO was studied in the Materials International Space Station Experiment (MISSE). The MISSE project started in 2001 and has tested over 4,000 materials during the projects lifetime [14]. The first eight missions were conducted by NASA, but now a company called Alpha Space is continuing the MISSE program. MISSE9 was the first MISSE mission conducted by Alpha Space with MISSE-10 and MISSE-11 currently waiting on the ISS to be installed [15][16]. Early tests consisted of a brief-case sized tray being placed on the ram side of the ISS with the materials facing normal to the ram direction. The newer tests conducted on Alpha Space's MISSE Flight Facility are held in modular containers that can be installed via robotic arm to remove the need for extravehicular activities by astronauts [14]. In each test were hundreds of materials for scientific, defense, and commercial study [17]. These on-orbit tests were the most comprehensive tests of AO's effects on materials. The downside to these on-orbit tests is the significant cost of bringing these pieces up onto the ISS. For this reason, materials and components are tested in labs on the ground as a precursor to testing them in space.

Materials are tested in simulated conditions on the ground to better understand and prevent this erosion. These test facilities follow the ASTM E2089 procedure for conducting their tests to ensure repeatability and to minimize variability between tests [18]. Unfortunately, not all test facilities are able to achieve the same environment to produce similar interaction mechanisms. Because of this, NASA has created documentation describing the different levels of AO tests [19]. In a level 1 test, the interaction mechanisms of the exposure environment with the material surface are different than the interaction mechanisms that would be experienced in LEO but, a level 1 test is required to contain oxygen and plasma systems [19]. One significant drawback of the level 1 test compared to 
others is that the AO does not impact the test with the same energy levels as AO in LEO [19]. This difference means that the eroded material will not have the pits and cones that are seen in material exposed to $\mathrm{AO}$ on-orbit. Instead the $\mathrm{AO}$ will erode the material evenly across its surface [19]. In a level 2 test the interaction mechanisms of the exposure environment is required to be fundamentally similar to the interaction mechanisms of $\mathrm{AO}$ on satellites in LEO without the synergistic effects of VUV light and ions [19]. This level of test is much more difficult to achieve as being fundamentally similar requires impinging AO to have an impact energy between 1.5 and $6.0 \mathrm{eV}$ as well as impacting within $\pm 10^{\circ}$ of normal to the sample surface [19]. A level 3 test is the most realistic of all the tests as it requires the exposure environment to be fundamentally similar to the environment that the material experiences with regard to AO in LEO, with the addition of the synergistic effects of VUV light and ions [19]. This test is the most difficult as not only do the particles of AO need to be accelerated to impact the test material at orbital velocities, it must also include synergistic effects [19].

\subsection{Patch Antenna}

Since 1999, there has been a noticeable increase in the number of small sized satellites being launched into orbit due to the invention of CubeSats by Dr. Jordi Puig-Suari and Dr. Robert Twiggs [20]. CubeSats are a type of smallsat with a defined form factor that allows for universities and startup companies to get to space at a much lower cost and in a timelier manner than traditional satellites. CubeSats are much smaller than traditional satellites, and can hitchhike on launches to orbit making them much cheaper to launch. Usually a CubeSat is limited in its available volume and must be selective about the payloads and equipment they take to orbit. For instance, A $1 \mathrm{U}$ CubeSat is currently limited to being $1.33 \mathrm{~kg}$ in mass 
and $10 \times 10 \times 10 \mathrm{~cm}$ in volume [21]. A patch antenna allows CubeSats to have a low profile, fixed antenna that does not run the risk of failing during its deployment. One of the leading causes of CubeSat failure is the communication system (COM) so eliminating any additional risks to the COM system is beneficial for the CubeSat reliability [22]. One of the major drawbacks in choosing a patch antenna for CubeSat missions is that the signal from a patch antenna is semi-directional [23]. This means that patch antenna cannot transmit or receive signal from a ground station or sister satellite if they are not orientated correctly. Monopole and dipole antennas meanwhile, are omnidirectional antenna meaning they can transmit and receive signal in any orientation. Although at first this seems to be a distinct disadvantage because the patch antenna is semi directional, it is less wasteful in the signal it transmits [23]. Rather than sending all of the signal in every direction it can focus its signal down to a $180^{\circ}$ swath, which can allow for higher data rates. For the purpose of this thesis, patch antennas were chosen for testing due to their cost, size, low profile, COTS availability, and low risk of failure compared to other deployable antenna.

A patch antenna is a metal sheet that can be rectangular or circular above a grounded substrate that is on top of a ground plane [24]. Often the antenna is made of printed circuit board with the substrate making up the antenna's dielectric material. The metal sheet or patch and the ground plane are able to form a resonant cavity which creates the desirable electromagnetic radiation into space. Figure 2.4 shows a cross section of a typical patch antenna. 


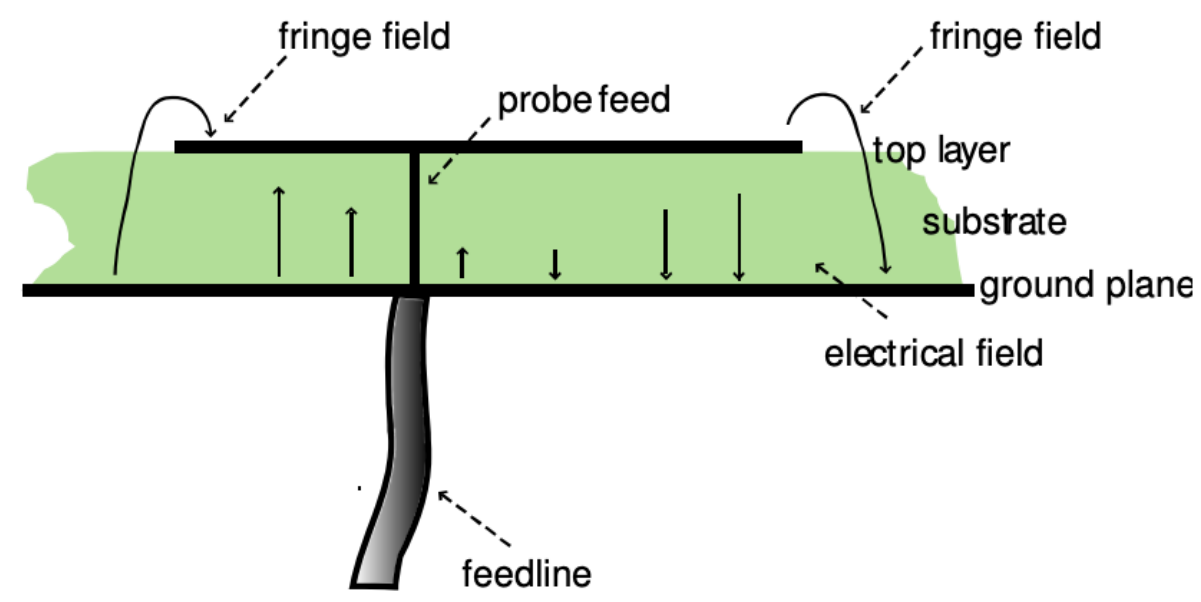

Figure 2.4: Cross Section of a Patch Antenna [25].

The bandwidth of the antenna is determined by the distance between the patch and the ground plane. The quality factor of the antenna is inversely proportional to the thickness of the patch antenna's substrate. This means that the bandwidth for a patch antenna is also proportional to the substrate thickness. The theoretical form of the electric field $(E(x))$ for a rectangular patch antenna is found thru equation 1 [24],

$$
E(x)=\cos \frac{\pi * x}{L}
$$

where $\mathrm{L}$ is the length of the dielectric and the $x$ is distance away from the center of the antenna in the electric field plane, and parallel to the surface of the patch. Typically, the width of the patch antenna is slightly larger than the length with a $\mathrm{W} / \mathrm{L}$ ratio of 1.5 . The quality factor $(Q)$ for a patch antenna is used to express the physical parameters of interest as a single comparable value and is defined using eq. 2 [24],

$$
Q=w_{0} * \frac{U_{s}}{P_{\text {in }}}
$$


where wo is the resonance frequency in radians per second, $U_{\mathrm{s}}$ is the energy stored in the patch cavity in joules, and Pin is the average power being supplied into the antenna in watts. Using the quality factor, the bandwidth can then be calculated using equation 3 [24],

$$
\text { Bandwidth }=\frac{S-1}{\sqrt{S} * Q}
$$

where $S$ is the standing wave ratio. The standing wave ratio is defined as the ratio of the maximum radio-frequency to the minimum radio frequency on a line such as a coaxial cable [24]. The standing wave ratio commonly used is 2 , to allow for some simplification of the previous equation [24]. One inherent disadvantage of patch antennas is that they have a small impedance bandwidth [26]. Bandwidth is the range of frequencies that an antenna can successfully radiate or receive signal at, so by having a small bandwidth, the range of frequencies that can be used with the antenna is limited. A lower bandwidth means that CubeSats will have a lower data rate. The radiation efficiency $\left(n_{e f f}\right)$ is used to measure the performance of the antenna and is a ratio of the power radiated into space versus the power input into the antenna as seen in equation 4 [24].

$$
n_{e f f}=\frac{P_{s p}}{P_{\text {in }}}
$$

Where $P_{s p}$ is the power radiated to space and $P_{i n}$ is the power supplied to the antenna. The inefficiencies in the antenna come from several sources - mainly dielectric losses, conduction losses, and surface wave losses [24]. These losses depend on the antenna design and can be minimized by various design decisions. For patch antenna, the resonant frequency is directly related to the dimensions of the antenna [24]. The resonant length is approximately one half of the resonant wavelength of the antenna when the patch antenna is excited in its fundamental mode [24]. 
Patch antenna can be used for a variety of communication functions. The four main functions are telemetry tracking and command (TT\&C), high-speed downlink of payload data, GPS/GNSS receiver to provide accurate locations of the satellite, and inter-satellite [23]. For the last of these functions, there has been an increase in interest as demand for a faster global communication service grows. This concept requires direct satellite to satellite communication. Although it does not use patch antennas, a prime example of this intersatellite communication can be seen in SpaceX's planned Starlink internet satellite constellation [27]. The constellation uses phased array beam forming in the Ku-band and Ka-band to communicate from satellite to satellite. For this application, it is inevitable that antenna will need to face in the ram direction of the spacecraft to be able to maintain communication links with the other satellites in its orbital plane. This will result in any antenna on the ram side of the spacecraft being exposed to AO particles impacting at orbital velocities. Inter-satellite communication can be very desirable for other missions as well. Another mission that makes use of patch antenna is the Gravity Recovery and Climate Experiment (GRACE) [28]. NASA's GRACE mission uses inter-satellite communication to help the payloads map variations in Earth's magnetic field [29]. The mission is composed of two satellites GRACE-1 and GRACE-2, and uses patch antenna to receive communication telecommands from the ground and has an additional transmitting patch antenna for redundancy. These antennas are used as the redundant antenna as they have very low pointing requirements due to a wide beamwidth and allow the spacecraft to communicate with the ground if a malfunction were to occur onboard that prevented the high gain antenna from establishing contact. The sister satellites also have horn antennas that are used to communicate directly between themselves. For the first part of the mission, 
GRACE-2 was flying in a "forward" orientation with its horn directly exposed to AO. Mission planners were concerned that exposure to AO could result in a loss of thermal control so after three and a half years the two satellites performed maneuvers to switch places with each other so the horn on GRACE-2 would no longer be impacted by AO [30].

Patch antennas are also used on CubeSats because of their low risk and low profile. Table 2.1 shows several CubeSat missions that have used patch antennas. One of the most successful instances of CubeSats that currently use patch antenna is the Planet Labs Dove constellation. This constellation currently consists of over 150 active CubeSats that comprise the largest satellite constellation to date [31]. These 3U Earth remote sensing satellites are capable of imaging the entire earth on a daily basis. Onboard each satellite is an S-band patch antenna operating with a central frequency at $2.4 \mathrm{GHz}$ and has a downlink rate of around $115 \mathrm{kbit} / \mathrm{s}$ [32]. Each of the Dove satellites also has a monopole antenna onboard for redundancy and to allow the satellite to receive and transmit signal. Figure 2.5 shows the Dove satellite with its S-band patch antenna.

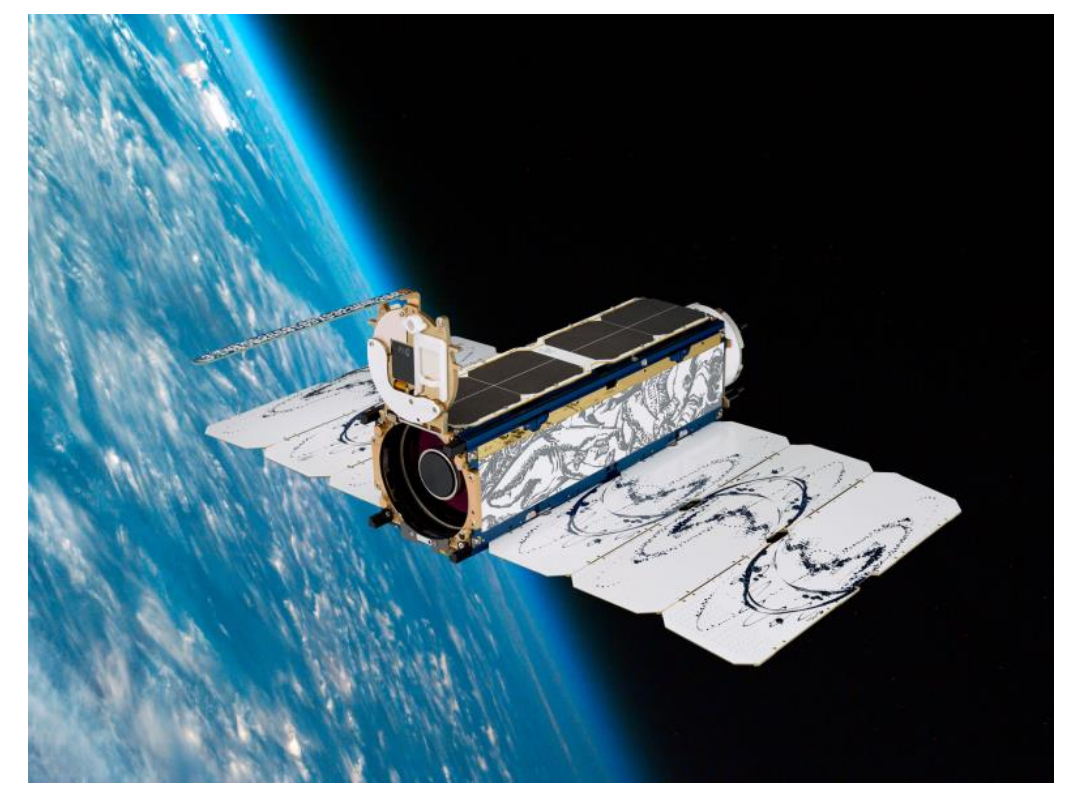

Figure 2.5: Dove Satellite with an S-band Patch Antenna [33]. 
Table 2.1: 2009-2012 CubeSat Missions Using Patch Antennas [34].

\begin{tabular}{ccccccc}
\hline Satellite & Size & Frequency & Power & Downloaded & Lifetime & Status \\
\hline AeroCube-3 & $1 \mathrm{U}$ & $915 \mathrm{MHz}$ & $2 \mathrm{~W}$ & $52 \mathrm{MB}$ & 7 months & Deorbited \\
PharmaSat & $3 \mathrm{U}$ & $2.4 \mathrm{GHz}$ & $1 \mathrm{~W}$ & $650 \mathrm{kB}$ & 10 days & Dead \\
Hayato & $1 \mathrm{U}$ & $13.275 \mathrm{GHz}$ & $100 \mathrm{~mW}$ & $0 \mathrm{kB}$ & 18 days & Deorbited \\
O/OREOS & $3 \mathrm{U}$ & $2.4 \mathrm{GHz}$ & $1 \mathrm{~W}$ & $8 \mathrm{MB}$ & $\begin{array}{c}29+ \\
\text { months }\end{array}$ & Alive \\
NanoSail-D2 & $3 \mathrm{U}$ & $2.4 \mathrm{GHz}$ & $1 \mathrm{~W}$ & $0 \mathrm{kB}$ & 5 days & Deorbited \\
Goliat & $1 \mathrm{U}$ & $2.4 \mathrm{GHz}$ & $1 \mathrm{~W}$ & $0 \mathrm{kB}$ & 1 week & Dead \\
AeroCube-4 & $1 \mathrm{U}$ & $915 \mathrm{MHz}$ & $2 \mathrm{~W}$ & $0 \mathrm{kB}$ & $8+$ months & Alive \\
CINEMA & $3 \mathrm{U}$ & $2200 \mathrm{MHz}$ & $1 \mathrm{~W}$ & $0 \mathrm{kB}$ & $8+$ months & Alive \\
\hline
\end{tabular}

Some of the missions in table 2.1 had both a patch antenna and a monopole antenna. This enables the CubeSat to receive and transmit signal in any orientation; when the patch antenna is oriented properly with another transmitting or receiving antenna, the patch antenna is capable of higher data rates. As CubeSats become more advanced, accurate attitude determination and control systems are necessary to point the satellite in a specific direction. The Dove 1 satellite for example uses magnetometers, photodiodes, and gyros to determine the CubeSat's orientation [32]. To control the satellite's orientation, Dove 1 also has magnetorquers and reaction wheels on board [31]. The magnetorquers and magnetometer are able to keep the satellite locked and axis aligned with the Earth's magnetic field and know the magnetic field to $1^{\circ}$ at any given time [31]. The pointing accuracy of the Dove satellite on-orbit was calculated to be $0.1^{\circ}$ [35]. This increase in attitude determination and control means that directional antennas, like patch antennas, can be used as long as the CubeSat meets their pointing requirement. Being able to use a 
directional antenna opposed to an omnidirectional antenna means the CubeSat can achieve a higher data rate with less power.

\subsection{Antenna Testing}

In the past, experiments related to AO erosion have focused heavily at how specific materials are effected by exposure to AO however, to the best of this author's knowledge, no studies have been found as to how these affects will change the performance of patch antenna onboard a spacecraft. An article on wide coverage antenna mentioned that a germanium coating could help prevent $\mathrm{AO}$ erosion on an antenna but did not expand on how that would affect performance or how long the coating would last for [36]. Typically, $\mathrm{AO}$ is not a major concern for spacecraft communication systems as the antenna is not typically facing in the ram direction of the spacecraft, nor did it have a significant area exposed to AO during the spacecraft's lifetime.

Functional tests are performed on COM systems to ensure the flight hardware complies with the system requirements for each satellite. Satellite functionality is checked after the COM system is exposed to vibration, shock, thermal, and acoustic testing to ensure the satellite meets launch system requirements. Vibration and shock tests are done to evaluate whether the COM system will survive the launch environment and deployment. Thermal vacuum testing is done to ensure the antenna can still meet performance requirements while operating at the thermal extremes of the mission. Acoustic testing is done to certify that the COM system will survive the acoustic pressures the satellite will experience during launch. These tests are done on the COM system both before and after they have been integrated onto the spacecraft [37]. After each test the gain, radiation pattern, polarization, and impedance are verified with manufacturer specifications. Although these tests attempt to 
ensure the survival of the antenna in the space and launch environment, they do not account for many other aspects of the space environment including exposure to AO.

To test the effects of $\mathrm{AO}$ exposure on patch antennas, the antennas first needed to have their gain and radiation pattern measured in an anechoic chamber. After the initial performance measurements, each antenna was exposed to $\mathrm{AO}$ in the $\mathrm{AO}$ test chamber. Each exposed antenna was then tested again in the anechoic chamber to watch for any changes in its performance characteristics. A significant number of antenna needed to be tested in order to verify the significance of any observed changes in antenna performance. To reach a confidence level of $85 \pm 15 \%, 24$ samples would need to be tested. After the tested patch antennas were exposed to the AO, a scanning electron microscope (SEM) was used to determine the material that was formed during the AO test. The SEM allowed for the material structure to be observed and with the help of a spectrometer, determine if the oxide formed is stable or unstable. Performance characteristics that were measured include gain and signal pattern, while the material properties observed were changes in surface roughness and material chemical composition.

\subsection{Antenna Selected}

To select an antenna for testing, several factors had to be considered to find a suitable antenna. First, the selected patch antenna needed to be small enough to fit both on a CubeSat and within the Cal Poly atomic oxygen test chamber. The antenna also needed to have suitable gain and frequencies so it would be comparable to current antennas used onboard CubeSats. Additionally, the patch antenna needed to be COTS with a wide availability as if the antenna were to be used by CubeSat developers in the future it would need to be easily available. Finally, the selected antenna needed to be inexpensive enough 
so it could be purchased and tested within the limited budget that was available for the thesis. Without having multiple antennas to test, it would be impossible to reach a result that had any apparent significance. After looking at many different types of patch antennas, the FXP72 Freedom 2.4 GHz Antenna made by Taoglass was selected for testing. At $31 \mathrm{x}$ $31 \times 0.1 \mathrm{~mm}$, the patch antenna is small enough to fit within the AO test chamber and on the face of a CubeSat. The FXP72 has a peak gain of $5 \mathrm{dBi}$ and operates in the S-Band from $2.3 \mathrm{GHz}$ to $2.6 \mathrm{GHz}$. The patch antenna itself is made of printed circuit board with a copper foil etched onto its surface and an exterior coating of polyamide. The cost per antenna was $\$ 3.44$ which is several orders of magnitude less than other patch antennas that are marketed specifically for use onboard CubeSats making it ideal for destructive testing. An image of the test antenna can be seen in figure 2.6 [38].

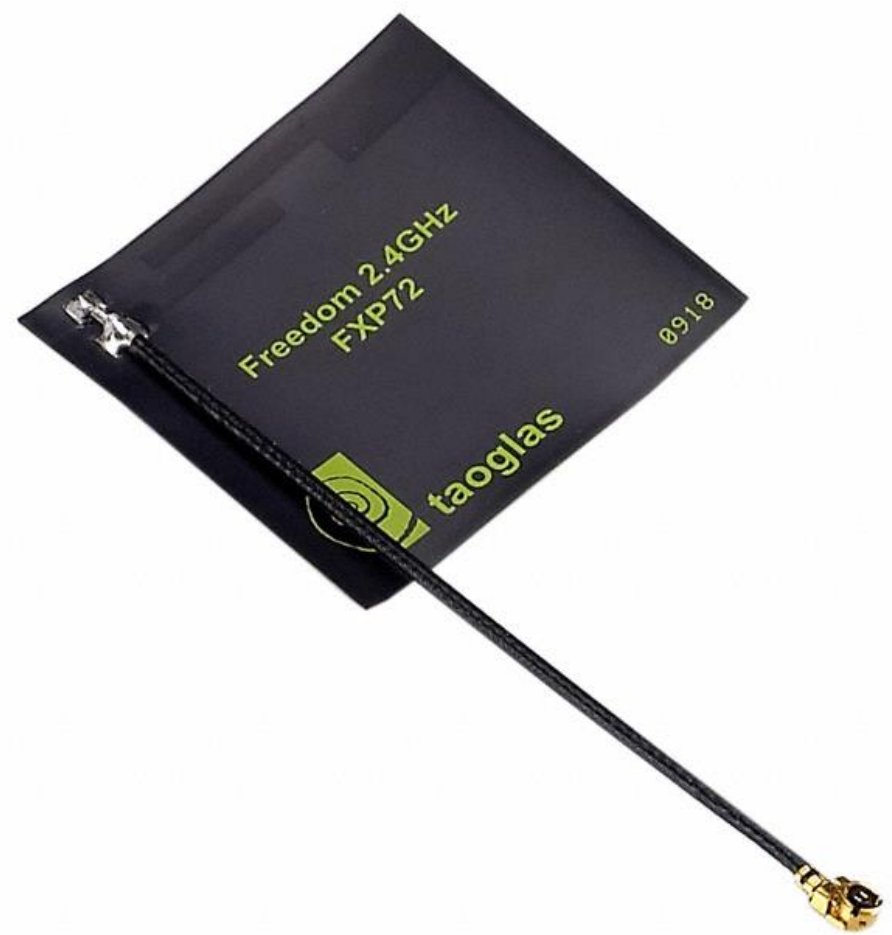

Figure 2.6: FXP72 Freedom 2.4 GHz Antenna Manufactured by Taoglass [38]. 


\section{Chapter 3}

\section{EXPERIMENTAL APPARATUSES}

This chapter covers the apparatuses used to conduct tests for this thesis. First is a description of the outgassing chamber Junior that was used to prepare samples. The chapter then discusses the AO chamber used to expose the test samples. The last apparatus discussed is the anechoic chamber that was used to measure antenna performance.

\subsection{Junior}

ASTM E2089 is the standard used to minimize variability in results for any given AO exposure facility [18]. To conform to the standards outlined in ASTM E2089 the test samples must be vacuum-dehydrated for at least 48 hours. This allows the material to outgas any absorbed water and prevent errors in mass measurements. Junior is a Kartell model DYNCR 243065 desiccator with a chamber of about $23.9 \mathrm{~cm}$ in diameter. The desiccator was purchased to have a dedicated chamber to outgas test samples for any students running AO tests. The pump used on Junior is a Cacejen Vacuum CVP 24 with an ultimate pressure of $5 \times 10-4$ Torr. Having a dedicated chamber to outgas samples in prior to running $\mathrm{AO}$ tests frees up other vacuum chambers for students in the Space Environments Laboratory. Previous work by Charles Ward has verified that the desiccator is capable of pumping down to approximately 188 mTorr which is below the required ASTM E2089 standard of 200 mTorr [39][18]. At 188 mTorr, Junior will be capable of properly outgassing samples of any absorbed water to provide for an accurate test. The vacuum chamber schematic for Junior is shown in figure 3.1 . 


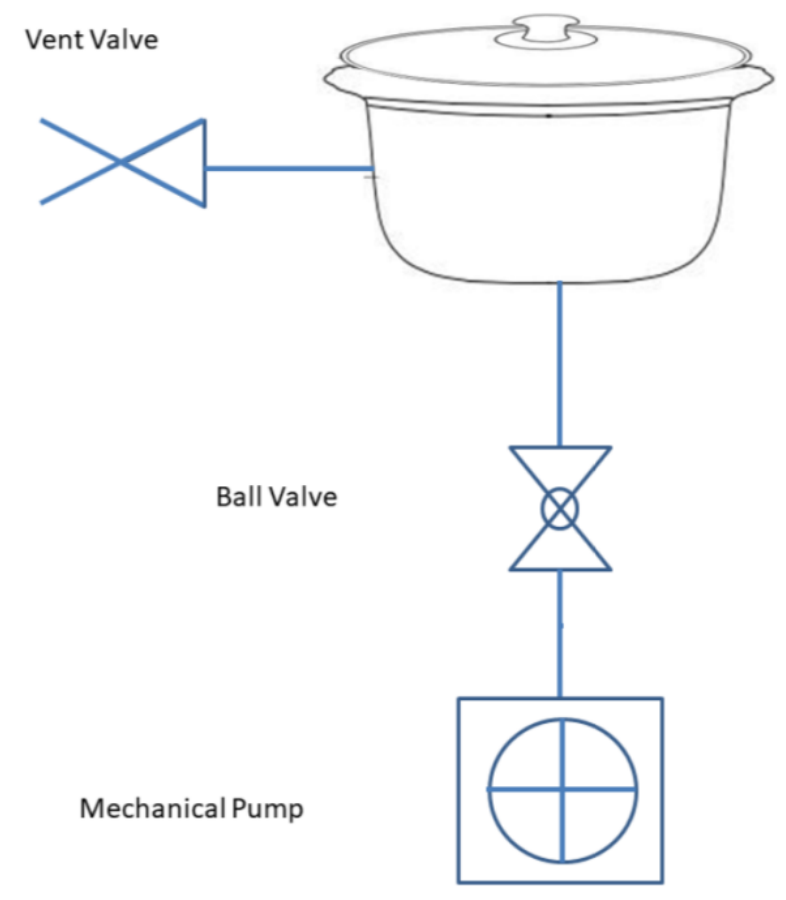

Figure 3.1: Junior Vacuum Chamber Schematic [39].

\subsection{MAX}

To expose the selected patch antenna to AO, the AO test chamber named the Minimum Atmospheric eXperimentation (MAX), in the Spacecraft Environments Laboratory at the California Polytechnic State University (Cal Poly), San Luis Obispo, was used. This chamber was built by Max Glicklin, an Aerospace Engineering Graduate Student from Cal Poly, for his master's thesis [40]. The MAX chamber is a capacitively coupled plasma asher system that creates a different environment than the $\mathrm{AO}$ environment found in orbit. This capacitively coupled plasma (CCP) system creates isotropic AO with thermal energies between $0.04-0.1 \mathrm{eV}$ [40]. The CCP system creates the AO by using two electrodes, one powered and one grounded with the powered electrode placed above the grounded electrode. When the system is operating an electric field forms between the upper, powered 
electrode and the lower, grounded electrode which ionizes atoms creating the plasma. The $\mathrm{AO}$ formed in this system differs from $\mathrm{AO}$ found in orbit as $\mathrm{AO}$ found in orbit is highly directional due to the high orbital velocities of LEO spacecraft whereas the isotropic plasma from a CCP system is omnidirectional. This means that any samples tested in MAX will not have the pits and cones that typically form from orbital AO exposure. Instead the AO erosion will occur evenly across the surface of the sample [41]. In an effort to set standards for material testing with AO, documents such as ASTM E2089 outline procedures for different types of $\mathrm{AO}$ chambers. For the $\mathrm{AO}$ exposure tests conducted in this thesis, ASTM E2089 was followed to ensure consistent and reliable testing setup. Another document created by NASA sets standards for different levels of AO test chambers which was discussed previously in section 2.1 [42]. Based on these standards the MAX chamber is a "Level 1 Testing" chamber. This means that even though the chamber will not be able to reproduce the exact same effects found in LEO, the results still carry some significance and warrant further study. After thorough testing, it was shown that the MAX test chamber is able to generate high AO flux values to allow for accelerated testing. A 24hour test in MAX generated a calculated AO effective fluence of $8.561 \pm 0.493 \cdot 10_{20}$ atoms per $\mathrm{cm}_{2}$, which is equivalent to roughly 3.4 months of on-orbit exposure at an orbit of 400 
$\mathrm{km}$ with an inclination of $28.5^{\circ}$ [8]. A schematic of a general CCP system is shown in figure 3.2 .

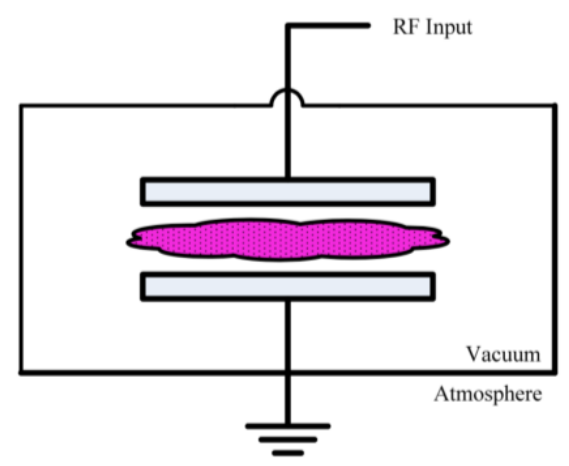

Figure 3.2: Simple Diagram of a Capacity Coupled Plasma Created in MAX [40].

To operate MAX, a comprehensive power supply system manufactured by Seren Industrial Power Systems is used. The specific radio frequency (RF) generator is a Seren R301MKII that operates at a fixed frequency of $13.56 \mathrm{MHz}$. The system has a maximum power output of $300 \mathrm{~W}$, however, for testing purposes the typical power supplied is 125 W. Previous tests in MAX have shown that increasing RF power levels beyond $125 \mathrm{~W}$ cause the samples and the ground plate to heat up to temperatures in excess of $100^{\circ} \mathrm{C}$ which is outside the survival temperature range of the selected antenna [40]. To protect the RF generator, a Seren IPS AT3 matching network is attached to the system. This device is designed to match the impedance load of the power generator and eliminate any reflected signals that are generated throughout the system. User input and a Seren MC2 controller, which adjusts the variable capacitors in the AT3 device to automatically match the impedance, control the system as a whole. For this chamber, the impedance load was 
selected to be 50 ohms per industry standards [40]. Figure 3.3 shows Seren R301MKII generator, and figure 3.4 shows the Seren IPS AT3 matching network and MC2 controller.

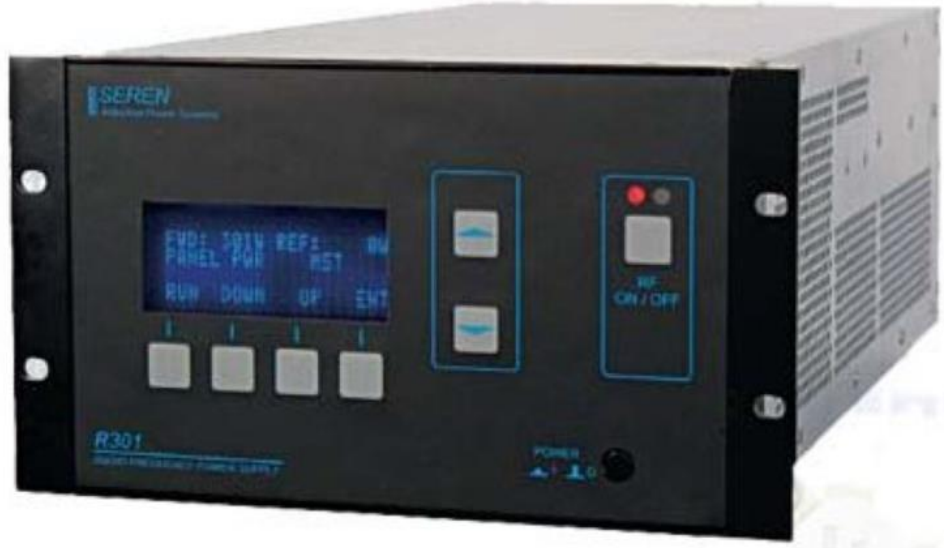

Figure 3.3: Seren R301MKII Generator Installed in MAX [43].
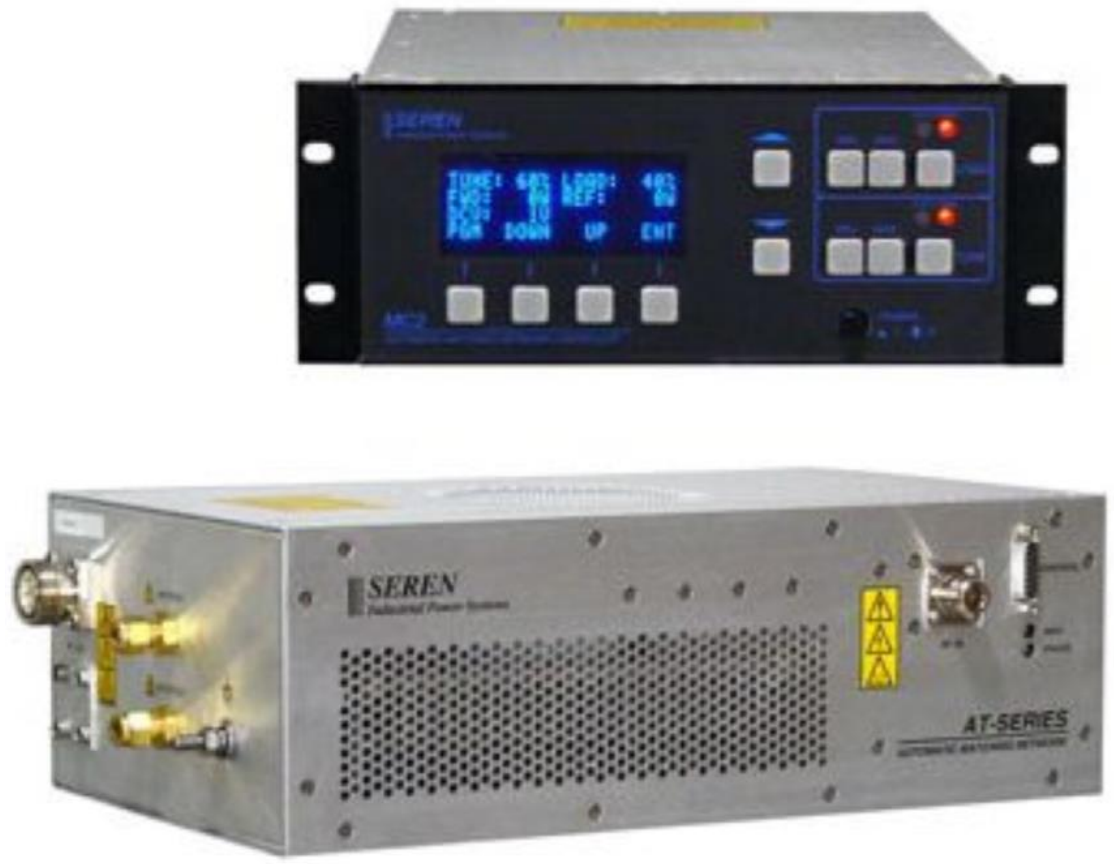

Figure 3.4: Seren IPS AT3 Matching Network (Bottom) and MC2 Controller (Top) [43]. 
The RF electrode used in the system is a $15.25 \mathrm{~cm}$ diameter by $0.9 \mathrm{~cm}$ thick disk of 6061 aluminum alloy which was selected due to the metal's relatively high sputtering threshold that minimizes contamination from the electrode itself [40]. The RF power connector is connected by a simple friction fit to the electrode. A dark space shield has also been installed to minimize secondary emissions from the electrode and to improve the AO in the desired region. A small gap of $1.9 \mathrm{~mm}$ exists between the dark space shield and the electrode, which is acceptable provided the gap remains less than the thickness of the plasma sheath [40]. This entire setup is attached to a hoist that allows for easy access to the interior of the chamber. The lower grounding electrode plate is a $25.4 \mathrm{~cm}$ square aluminum plate grounded with a copper strap. Currently there are two options to choose from for the lower grounding plate and depends on the size of the sample that is being exposed to AO. For the first grounding plate, each circular test sample hole has an equal radial and axial displacement from the RF electrode as well as an exposed surface area of $5.06 \pm 0.02 \mathrm{~cm} 2$. The second was designed as part of Charles Ward's thesis and consists of two parallel slots that measure 206 by $45 \mathrm{~mm}$ and are separated by $25 \mathrm{~mm}$ [39]. An image of the circular test sample hole grounding plate can be seen in figure 3.5 and an image of the trough plate can be seen in figure 3.6 . 


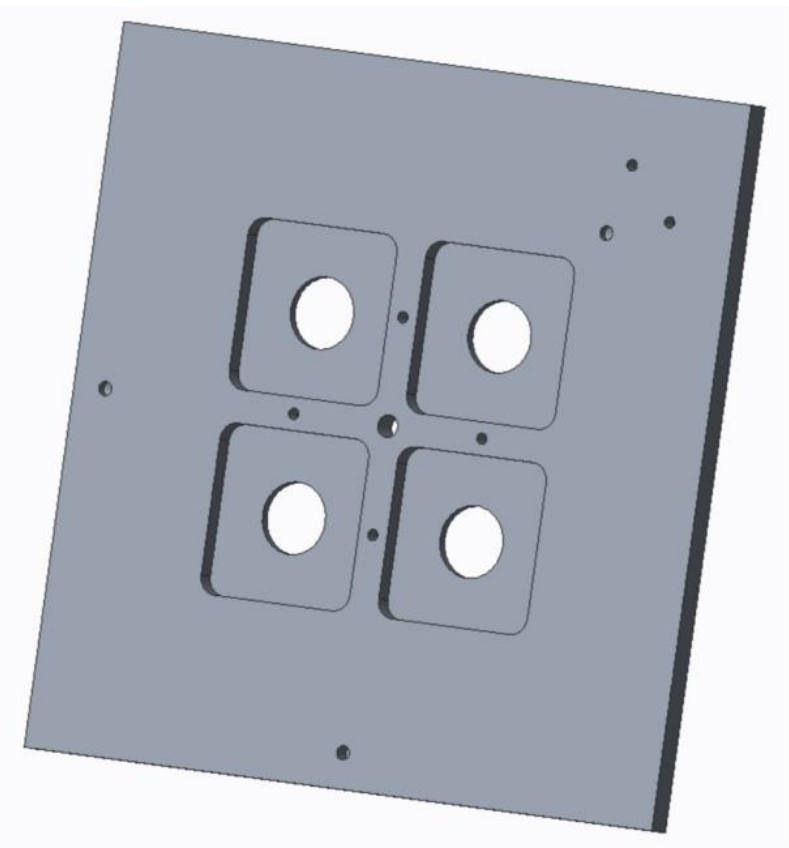

Figure 3.5: Thin Samples Grounding Plate [39].

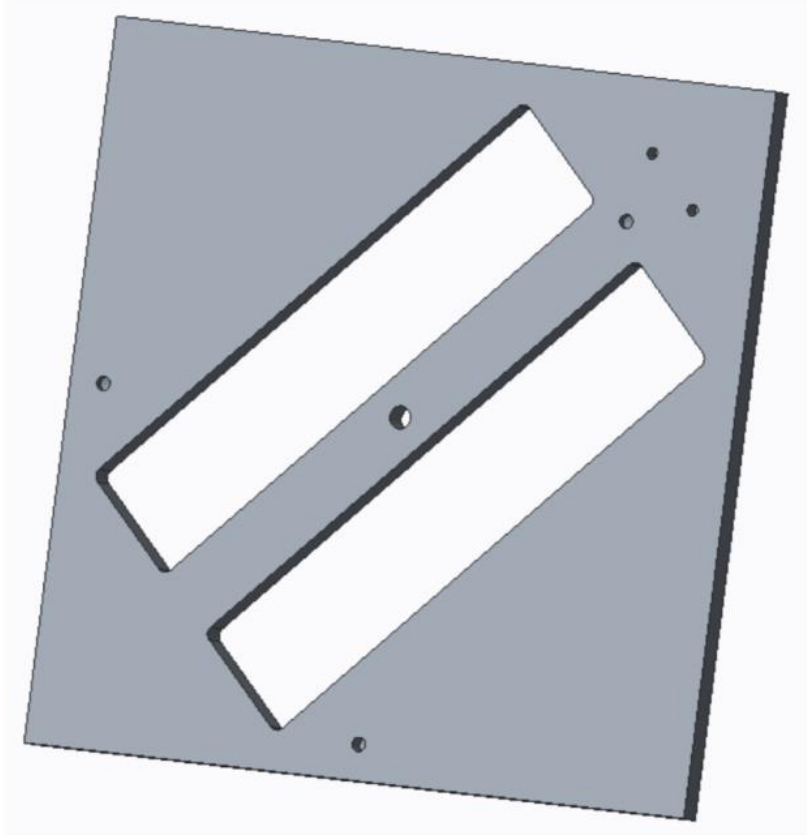

Figure 3.6: Two-Slot Grounding Plate [39].

For the majority of the tests, the grounding plate designed by Charles Ward was used as it allowed for a greater number of antennas to be tested at the same time. During testing, a 
gap distance between the two pieces of aluminum of $7.62 \mathrm{~cm}$ is used which previous tests determined as the optimal distance for creating AO [40]. For both grounding plates, a 0.8 $\mathrm{cm}$ diameter hole is cutout in the center to allow for air to be bled into the system. The air that is bled into the system, using a needle valve, allows for a continuous flux of $\mathrm{AO}$ on the test samples, and is fed into the center to allow for an even distribution of air to reach each of the test samples. Copper straps are used to ground the top and bottom square aluminum plates. An image of MAX can be seen in figure 3.7.

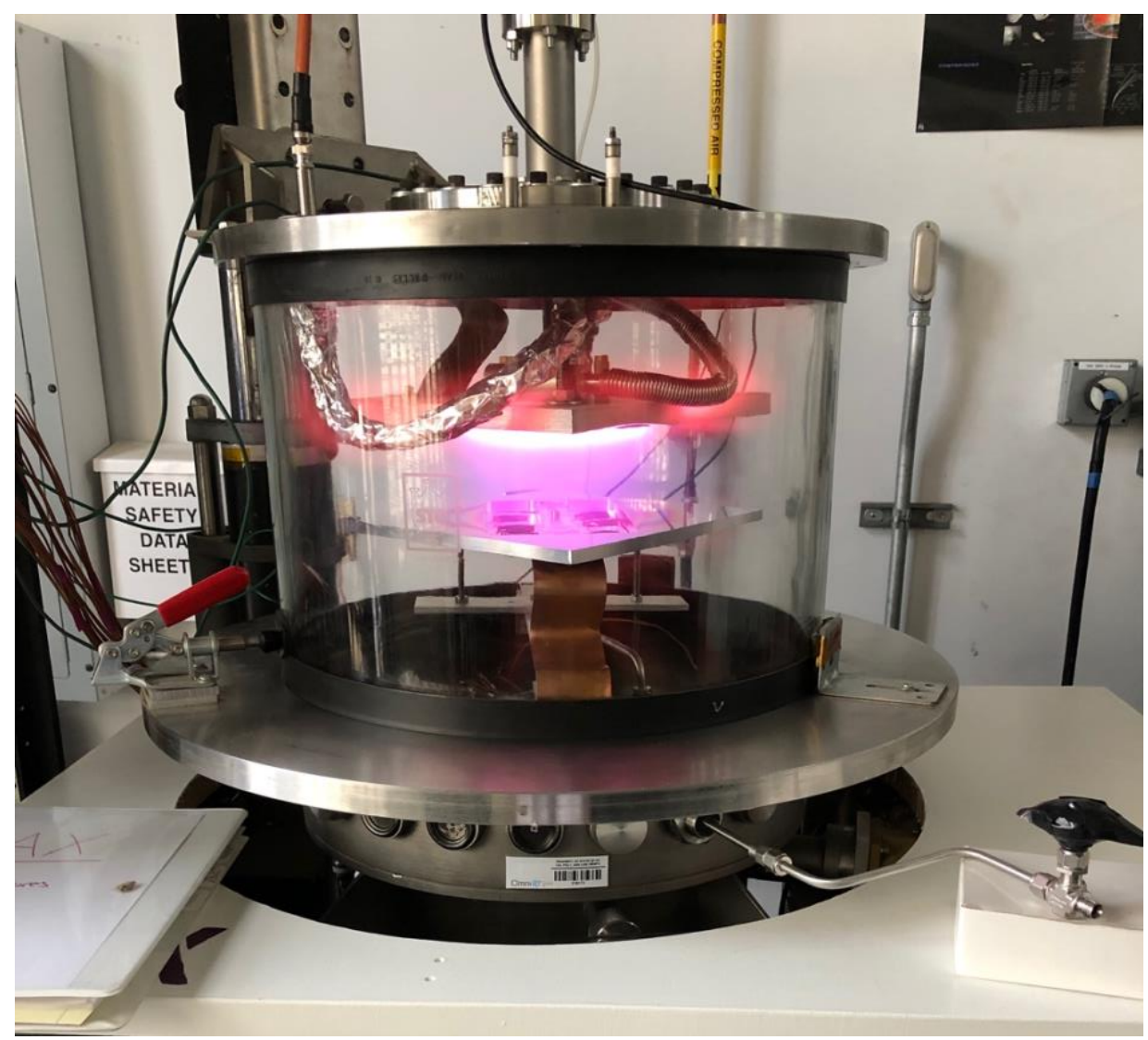

Figure 3.7: MAX Creating a Capacity Coupled Plasma to Produce Atomic Oxygen.

MAX's vacuum chamber is composed of a $50 \mathrm{~cm}$ diameter by $32 \mathrm{~cm}$ tall Pyrex cylinder that has been modified to be airtight. This year a new mechanical roughing pump was installed in MAX. The pump is an Adixen ACP 28, standard, single phase, manual gas 
ballast made by Pfeiffer Vacuum. The pump is capable of pumping down to an ultimate pressure of 22.5 mTorr and has a maximum flowrate of $410 \mathrm{~m} 3 / \mathrm{h}$ which is easily low enough for MAX to produce AO. To maintain a low vacuum pressure of $175 \pm 10$ mTorr, the ACP 28 mechanical pump is continuously run throughout the duration of the test. To allow air to enter the chamber, a needle valve is opened which allows a small amount of air to continuously enter the chamber. To balance the pressure, it was necessary to adjust the needle valve at the beginning of each test to balance the volumetric flow of air in and out of the chamber. A diagram of the chamber can be seen in figure 3.8.

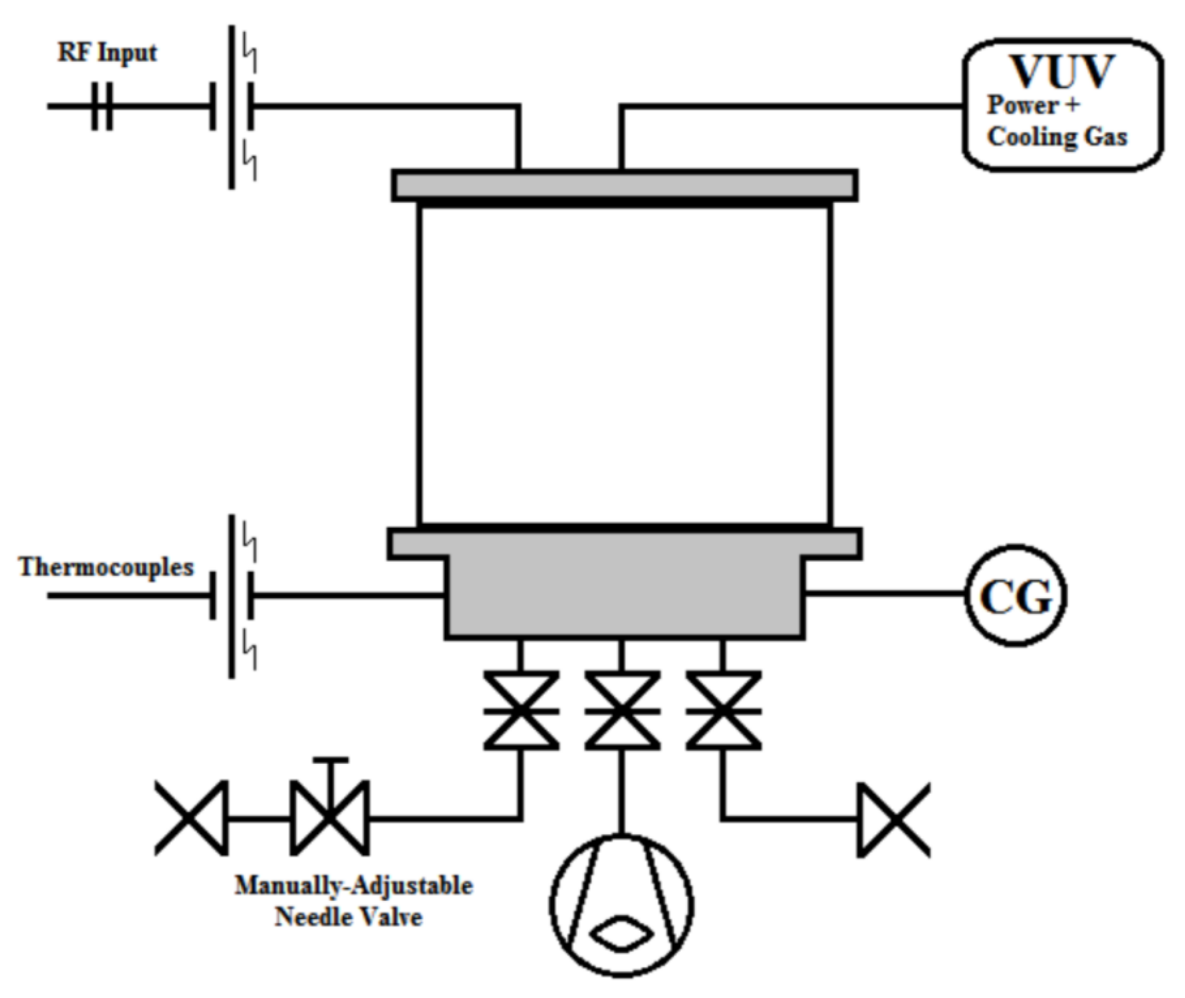

Figure 3.8: MAX Chamber Schematic [44].

During the 48-hour tests that were conducted, it was necessary to observe the temperature of the grounding plate as there was concern that the plates would heat up to above the antenna's operational temperature. To enable these temperature readings, two 
thermocouples were attached to the bottom and top of the ground plate with Kapton tape. To ensure accurate readings during each measurement, the power of the AO system was turned off briefly whenever temperature readings were taken. The temperature was taken with an Omega HH85 Thermometer that was connected to the thermocouple with the wire being fed into the chamber through an electric current feedthrough.

\subsection{Anechoic Chamber}

Cal Poly has two anechoic chambers that are able to measure the gain and signal pattern produced by these antennas. An anechoic chamber is a room that has been shielded to prevent outside RF sources from entering and to keep RF signals produced in the chamber from escaping [45]. For this experiment, the anechoic chamber used is the Cal Poly Microwave Lab Anechoic Chamber managed by Dr. Dean Arakaki. This chamber has been used previously to test antenna by several different companies including Cubic Defense Applications, AeroMech Engineering, and Stellar Exploration. Stellar Exploration even used the chamber to test a wrap-around patch antenna that was to be used for relaying communications between ground stations and sounding rockets. The anechoic chamber is 6.096 meters by 3.048 meters by 2.946 meters and has four panels that can be opened to access the chamber. Inside the chamber, the walls are lined with pyramidal absorber foam to block any exterior RF interference. The anechoic chamber is capable of measuring radiation patterns and the $S_{21}$ parameter which allows for gain to be calculated. The $S_{21}$ parameter represents the power transferred from port 1 to port 2 . To calculate the gain of the test antenna, two standard gain horn antennas with known parameters were used to act as reference antennas. The two standard gain horn antennas used in the anechoic chamber were a Microlab Model S438A S-Band Horn and a Narda Model 644 Standard Gain Horn. 
The process of testing antenna gain can be seen in appendix A. A cross-sectional view of the anechoic chamber can be seen in figure 3.9. The Mircolab horn antenna was used as the transmitting antenna and was used for every antenna tested. The Narda horn antenna was used as the reference antenna for the antennas under test (AUT). This reference antenna is placed in the receiving antenna position and connected to the vector network analyzer which measures the $S_{21}$ parameter of the antenna. Because the performance specifications of the Narda horn antenna are known it is possible to use the measured $\mathbf{S}_{21}$ value as a reference to calculate the true gain of the AUT.

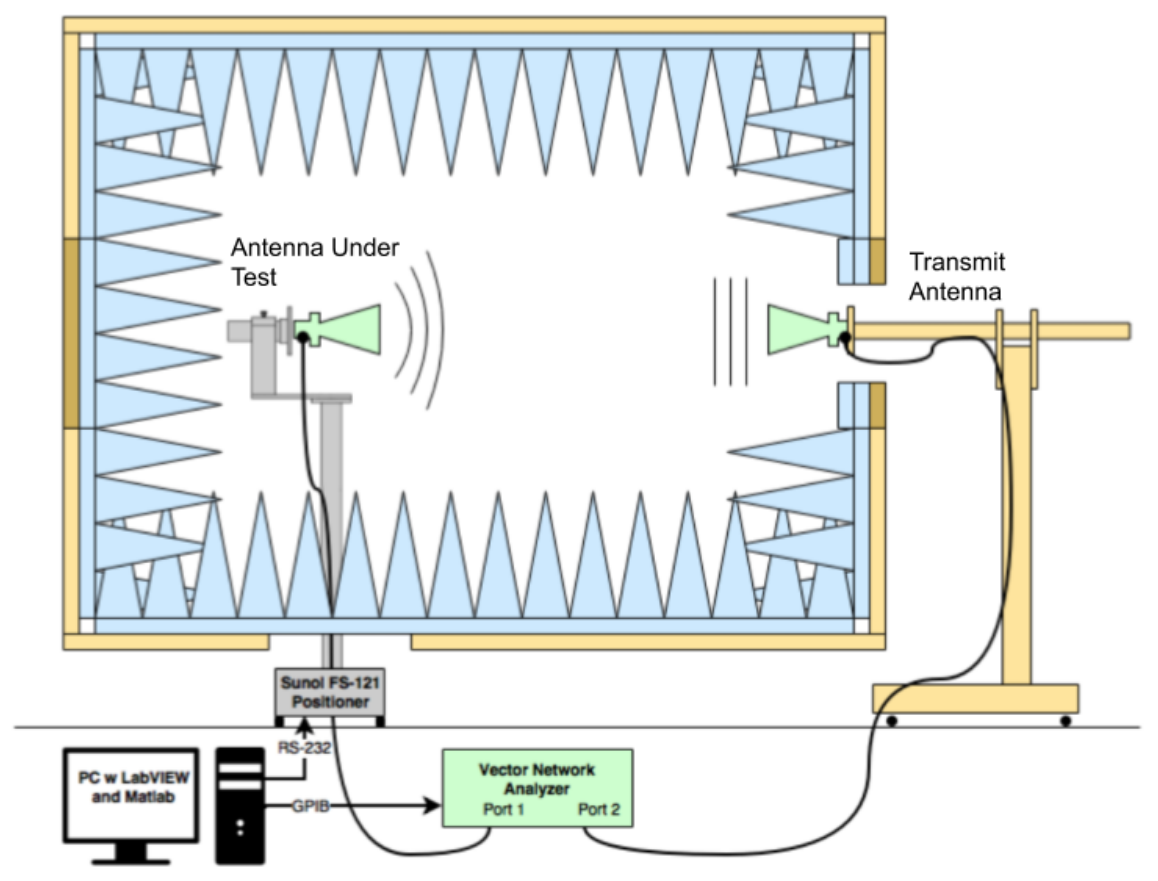

Figure 3.9: Cal Poly Microwave Lab Anechoic Chamber Cross Section [46].

To measure the gain and radiation pattern of the patch antennas both the transmitting Microlab horn antenna and the patch antennas were connected to an HP 8720C Network Analyzer. This vector network analyzer (VNA) works similar to other VNAs by using a 
source and a set of receivers to measure changes in the signal. The VNA's source signal frequency can range from $50 \mathrm{MHz}$ all the way up to $20 \mathrm{GHz}$ and has a frequency resolution of $100 \mathrm{kHz}$. If needed, the VNA has the ability to reach a frequency range of $1 \mathrm{~Hz}$. However, for most applications including the tests conducted in this thesis, it is unnecessary since it would not show a discernable difference in the measurements. During the testing for this thesis, the VNA sends a signal to the transmitting Mircolab horn antenna that is then received by the AUT. The AUT then sends the signal back to the VNA receivers where the returning signal strength is measured [47]. A diagram of this process can be seen in figure 3.10 .

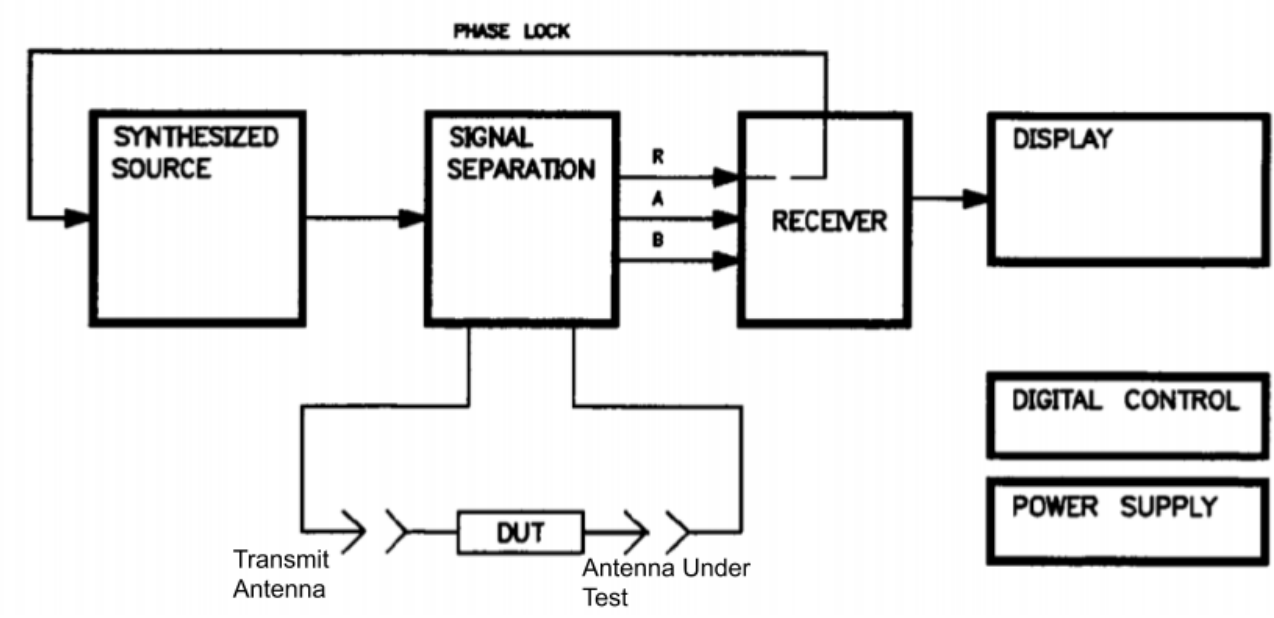

Figure 3.10: Block Diagram of HP8720C Network Analyzer During Operation [47].

To test the patch antenna in the anechoic chamber a special mount had to be constructed to hold the antenna in place in the chamber and allow for faster, consistent testing. The original mount in the chamber required the tested antennas to be bolted to its surface and since the patch antennas could not be attached in that manner a new mount was constructed. The mount was made by using four layers of clear acrylic as the plastic is transparent to radio frequency transmissions. The mount was built so that one of the patch antennas could 
be easily slipped into place and tested without having to completely remove the mount from the inside of the anechoic chamber. Each layer of the mount was laser cut in the $\mathrm{Cal}$ Poly Bonderson Project Center. An image of the mount can be seen in figure 3.11.

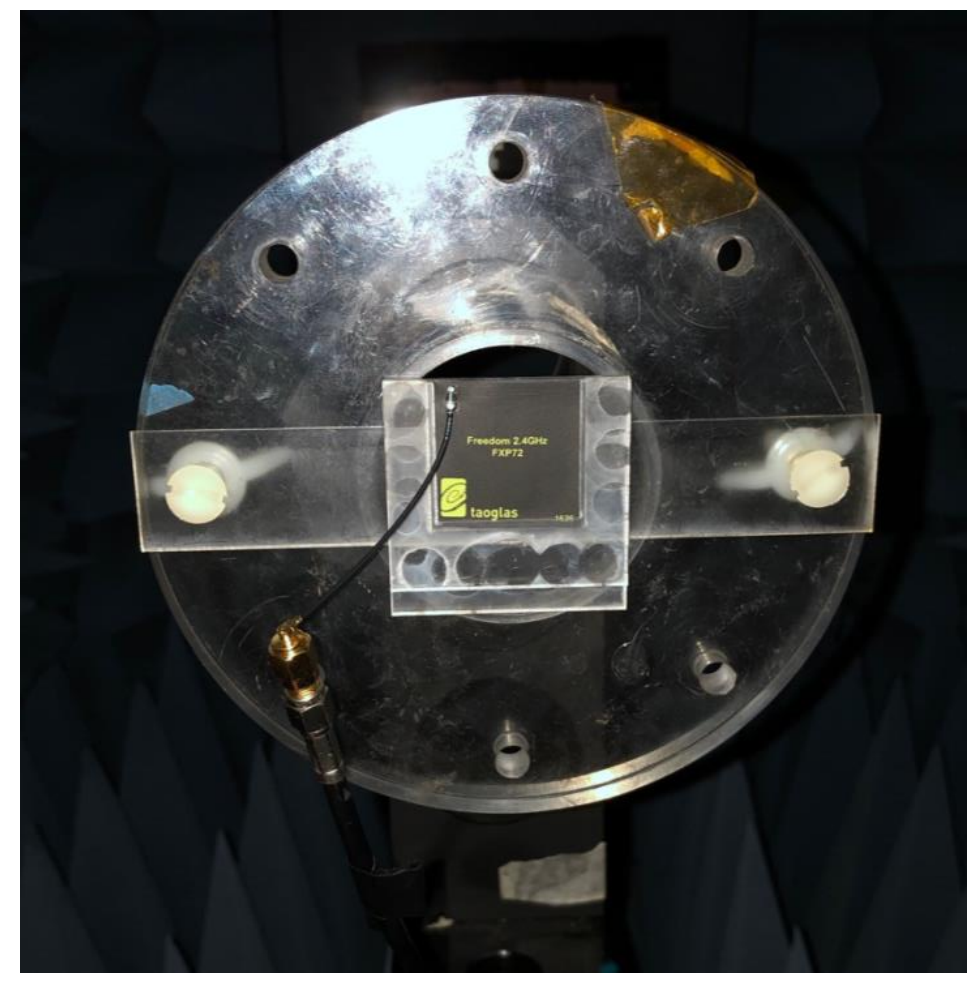

Figure 3.11: Patch Antenna in the Acrylic Mount.

During testing, the mount was placed on the ELAZ75 Positioner System, which is controlled by the SC104V Positioner Controller. This system allows for the antenna to be rotated $360^{\circ}$. To fully map the radiation pattern of the antenna it is necessary to map both the E-plane and the H-plane, which for the selected patch antenna are $90^{\circ}$ out of phase. The positioner can only rotate around one axis, so to measure both the E-plane and H-plane radiation pattern, the AUT must be rotated $90^{\circ}$ after measuring the H-plane radiation pattern [48]. The positioner is connected to a computer where the LabView Antenna Measurement System (AMS) Network Analyzer Application is used to direct the antenna 
to a specific angle. Figure 3.12 shows the patch antenna in its mount with the E-plane and H-plane. The E-plane and H-plane are both orientated with respect to the antenna.

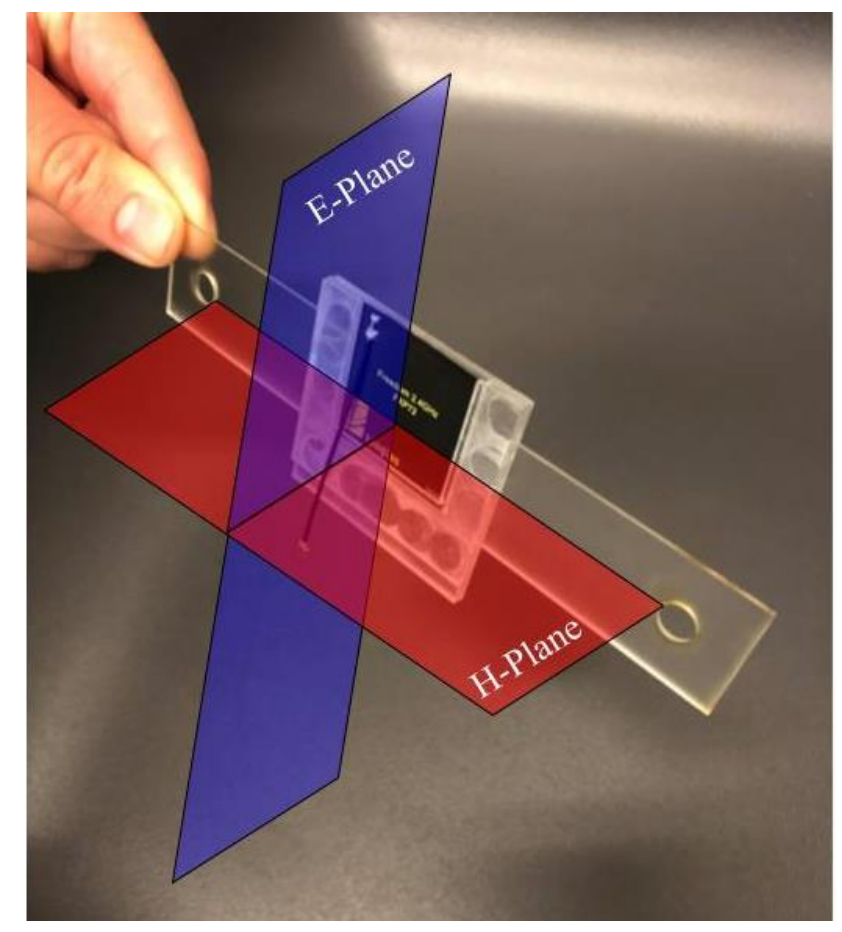

Figure 3.12: Patch Antenna in Mount with E-plane and H-plane.

Because the selected patch antenna was a linearly polarized antenna that means that these two planes are $90^{\circ}$ out of phase with one another. To help understand the difference in the E-plane and H-plane please refer to figure 3.13 of the antenna with a coordinate frame where the $\mathrm{X}$-axis is normal to the surface of the patch antenna. The E-plane is the plane in the $\mathrm{XZ}$ plane and the $\mathrm{H}$-plane is in the $\mathrm{XY}$ plane. 


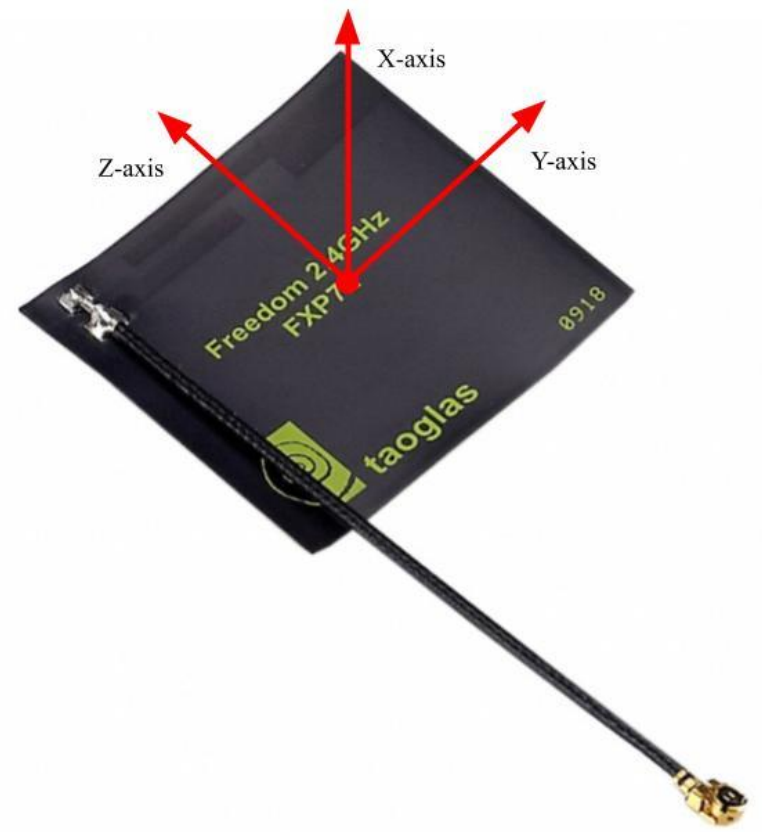

Figure 3.13: Patch Antenna with Coordinate Frame [38]. 


\section{Chapter 4}

\section{EXPERIMENTAL TESTING}

This chapter describes the various testing done on the antennas. The chapter starts with the testing done in the anechoic chamber and then describes the $\mathrm{AO}$ exposure testing conducted on the antenna samples. The last part of the chapter discusses the process and instruments used to identify the antenna surface material.

\subsection{Antenna Performance Testing}

Before the patch antenna could be exposed to $\mathrm{AO}$, the gain and radiation pattern of each antenna needed to be taken. This step was taken to prevent any variation in performance between antenna from being misconstrued as a change due to $\mathrm{AO}$ erosion. At the start of each round of performance testing the reference Narda Model 644 Standard Gain Horn antenna had its peak gain measured to use as a reference for calculating the gain of the patch antennas. The equation for calculating the gain $\left(G_{a}\right)$ of the patch antennas using a reference antenna can be seen in equation 5 .

$$
G_{a}=G_{s}+S_{21}^{a}
$$

Where $G_{S}$ is the gain of the reference antenna and $S_{21}^{a}$ is the measured value of the antenna under test. After measuring the peak gain of the reference antenna, the patch antennas could then be tested. The custom acrylic mount was mounted onto the positioner and the antenna was inserted into the mount. The SMA cable was then connected to the antenna via an SMA to U.FL adapter in such a way as to minimize any torque on the connection point or coaxial cable. After the patch antenna was successfully mounted, leveled, and pointed at the transmit antenna the anechoic chamber was closed, and measurements could be taken. The gain was measured first over the frequency range of 
2.39 to $2.49 \mathrm{GHz}$ followed by the radiation pattern at $2.44 \mathrm{GHz}$. These frequencies were chosen as they matched the frequencies used by Taoglass on the product datasheet [38]. To measure the radiation pattern, the patch antenna had to be rotated around its E-plane also known as the electric field plane as well as the H-plane, which is the magnetic field plane. The radiation pattern for both the E-plane and the H-plane were done for a full $360^{\circ}$ in steps of $5^{\circ}$ because initial tests using $1^{\circ}$ steps showed no improvement in radiation pattern shape.

\subsection{Atomic Oxygen Testing}

The atomic oxygen testing was conducted per the specifications stated in ASTM E2089 [18]. These specifications were followed to make any of the tests conducted during the experiment easily repeatable. Because the antennas tested in the anechoic chamber were backed with a sheet of wax paper, it was removed prior to testing and replaced with Kapton to minimize any $\mathrm{AO}$ erosion to the back of the antenna. If the patch antennas were to be used on a CubeSat the back of the antenna would be adhered to one side of the CubeSat and would therefore never be directly exposed to AO. To prepare the samples for AO exposure per ASTM E2089 standards the patch antennas were outgassed for 48 hours in Junior. After recording the post bakeout mass the samples were then ready to be placed in the AO chamber. The procedure for the tests run in MAX can be found in appendix B. In ASTM E2089 two key parameters are outlined as necessary for determining the extent of $\mathrm{AO}$ exposure during a test. Flux is the number of atoms per square centimeter per second and can be calculated by using Equation 6 where $f$ is the effective flux, $\Delta \mathrm{M}$ is equal to the change in mass, $\mathrm{A}$ is the area in square centimeters, $\rho$ is the density of the material, $\mathrm{E}$ is the reaction coefficient of the material, and $t$ is the time in seconds. 


$$
f=\frac{\Delta \mathrm{M}}{A * \rho * E * t}
$$

Fluence, on the other hand, is the total number of atoms per square centimeter and can be seen in equation 7 where $F$ is the effective fluence.

$$
F=\frac{\Delta \mathrm{M}}{A * \rho * E}
$$

For every test, it was required that a witness sample of Kapton be included to determine the flux and fluence seen on the samples during the test. Polyimide Kapton $\mathrm{H}$ was used because the reaction coefficient is known and the interaction between it and $\mathrm{AO}$ has been highly studied over the years. The trough plate does not have a designated location for a witness sample that prevents $\mathrm{AO}$ from eroding the underside of the witness sample. To minimize erosion on the underside of the Kapton witness sample, its edges had to be pressed down with aluminum tape. This was done by placing the witness sample on the bridge of aluminum between the two troughs, and taping 3 edges while still leaving the middle of the sample exposed to AO. An image of this setup can be seen in figure 4.1.

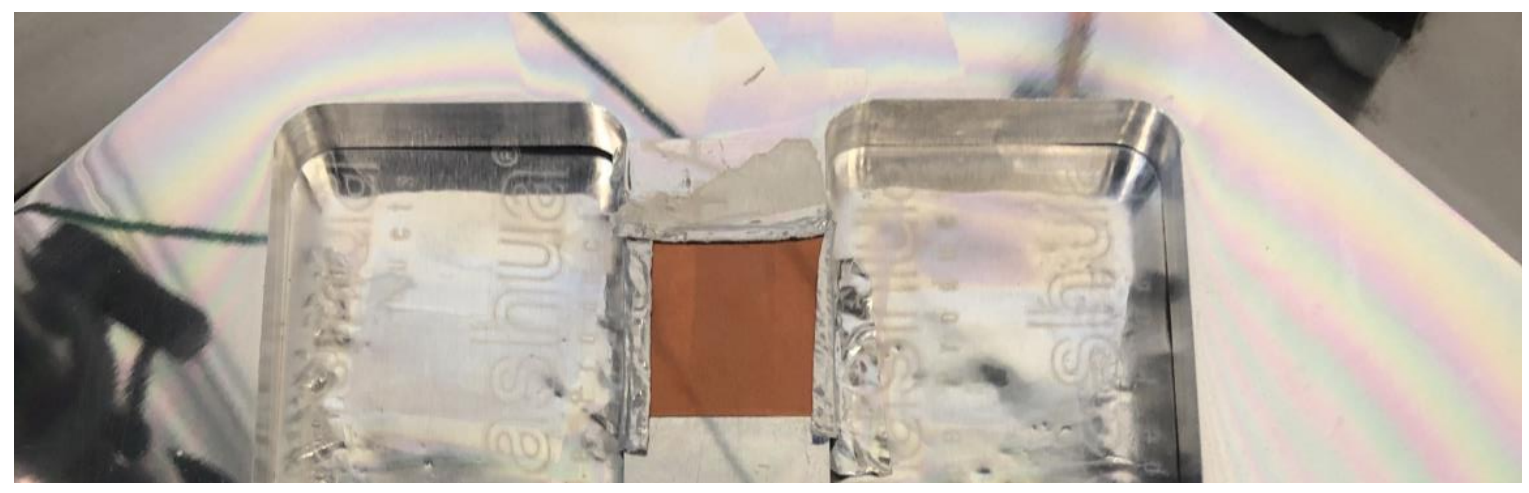

Figure 4.1: Kapton Witness Sample with Edges Sealed by Aluminum Tape.

After each test, an image processing software called ImageJ was used to determine the total area of exposed Kapton [49]. It is noted in ASTM E2089 that the effective flux and 
fluence are estimates and not the true values as the true values would be impossible to measure in MAX since a true value would require every molecule of AO to be recorded when it hit the witness sample. Previous experiments have shown that this chamber is capable of simulating 3-6 months of on-orbit AO exposure on the Ram side when in a $28.5^{\circ}$ inclined orbit with an altitude of $400 \mathrm{~km}$, depending on the length of the test that is run [8]. While conducting tests in MAX, the control antennas were stored in sealed Tupperware and not under vacuum. After testing, it was realized that because the control antennas were not kept under vacuum for the same duration as the samples in MAX, a difference in performance could have been attributed to either AO erosion or longer exposure under vacuum. It is possible, but unlikely that additional time under vacuum would cancel out the effects of AO. The additional time under vacuum would allow more time for outgassing, however the antennas were outgassed prior to AO exposure and exposed to atmospheric pressure before being tested in the anechoic chamber. It should also be noted that the erosion seen in these tests will not be exactly the same as erosion from on-orbit AO exposure as the chamber currently cannot reproduce many of the synergistic effects that occur on-orbit such as AO and UV radiation or the high kinetic energy impacts of the AO molecules on the sample materials.

\subsection{Scanning Electron Microscope}

In an effort to observe the erosion happening on the surface of the patch antenna, an FEI Quanta 200 Environmental Scanning Electron Microscope (SEM), owned by the Cal Poly Material Engineering department, was used to observe the surface of the patch antenna before and after 48 hours of AO erosion. The SEM produced its electron beam using a tungsten filament and captured the images using a large field detector. This 
particular SEM is capable of pumping down to 10-7 Torr however for this test it was only pumped down to a low pressure vacuum of $0.750-0.975$ Torr. The reason the antennas were observed under low vacuum is because the surface is made of non-conductive material, so in order for the materials to be seen by the large field detector, water molecules must be injected into the chamber. The water molecules then hit the surface of the sample and become ionized by the electrons. After colliding with the sample, the water molecules are forced off the sample causing electrons to hit the large field detector and create an image [50]. To image the patch antenna prior to $\mathrm{AO}$ exposure an accelerating voltage of $13 \mathrm{kV}$ was used with a spot size of 4.0. After 48 hours of AO exposure the patch antenna was imaged with an accelerating voltage of $16 \mathrm{kV}$ and a spot size of 4.0, where the spot size is the size of the electron beam when the beam hits the surface of the sample [50]. The reason for the difference in accelerating voltage is discussed later in this thesis.

\subsection{Fourier Transform Infrared Spectroscopy}

Taoglass was not willing to say the exact material used as the coating for the patch antennas, but they were willing to say that the material was a polyamide. To determine what the unknown polyamide was and how it was interacting with AO, Fourier Transform Infrared Spectroscopy was conducted on a control patch antenna for both the 24-hour and 48-hour exposure tests. The apparatus used was the Cal Poly Materials Engineering department's JASCO FTIR 4600 Spectrometer, set in the attenuated total reflectance (ATR) mode. The machine sends a beam of infrared radiation through a single beam optical system and measures the transmitted light using a detector. When the IR radiation hits the sample material it vibrates the bonds causing them to stretch or bend depending on the type of bond, frequency, and intensity of the absorption peak [51]. The resulting signal is 
measured at each wavenumber and a spectral fingerprint is formed for each material. This spectral fingerprint can then be used to help identify the material such as in this case where it is suspected that the material is a polyamide. Additionally, the FTIR can easily show any changes of chemical bonds on the surface of the patch antennas after they have been exposed to $\mathrm{AO}$, as a change in chemical bonds will result in a change in the absorption peaks seen in the sample. The JASCO 4600 FTIR spectrometer uses a diamond prism and has a maximum resolution of $0.7 \mathrm{~cm}-1$ [52]. 


\section{Chapter 5}

\section{RESULTS}

This chapter discusses the results of the tests conducted, beginning with the testing done to verify the AO chamber was still functioning as it had before. Next the 24-hour AO exposure antenna testing results are discussed followed by the results of the 48-hour AO exposure antenna testing. The chapter ends with the results of the spectroscopy and SEM imaging.

\subsection{MAX Verification}

Since MAX has been used for many different experiments in the past and still runs consistently today, there was not much verification testing required to prove that MAX can conform to ASTM E2089. An initial 24-hour test was done at the start of the year to compare flux and fluence values of the chamber and were found to conform to values seen in previous tests. The four-sample plate was used to hold four witness samples to measure the effective fluence, which was calculated to be $1.42 \pm 0.05 \cdot 10_{21}$ atoms $/ \mathrm{cm} 2$, just below the expected fluence of $1.47 \cdot 10_{21}$ atoms $/ \mathrm{cm}_{2}$ [40]. Since the plate designed by Charles Ward was used for testing of the antennas, a test was conducted with samples of Kapton placed in the same spots that the antennas were placed in to observe any differences in AO flux and fluence. The difference in the flux and fluence values seen for each sample along with its location can be seen in table 5.1. Figure 5.1 shows the positions each sample was placed in for each AO test. It should be noted that the flux and fluence values for these samples are from only one test. More tests are needed to discern if the results are significant, but it was beyond the scope of this thesis. To calculate the error in the flux and fluence for each sample, many factors were taken into consideration including the error in 
the calculated exposed area, the error in the mass measurements, the error in the density of the Kapton, and the error in the reaction coefficient of the Kapton. To accurately propagate error different methods must be used when adding and subtracting versus multiplying and dividing. Equation 6 was used to propagate error from addition and subtraction [53].

$$
\delta Q=\sqrt{\delta a^{2}+\cdots+\delta z^{2}}
$$

Where $\delta Q$ is the total error of numerical value $Q, \delta a$ is the error of numerical value $a$, and $\delta z$ is the error of numerical value $z$. The method for propagating error through multiplication and division is shown in equation 7 [53].

$$
\delta Q=|Q| * \sqrt{\left(\frac{\delta a}{a}\right)^{2}+\cdots+\left(\frac{\delta z}{z}\right)^{2}}
$$

This method was used to calculate the error for the effective flux and fluence in each test and came from a Harvard paper on error propagation.

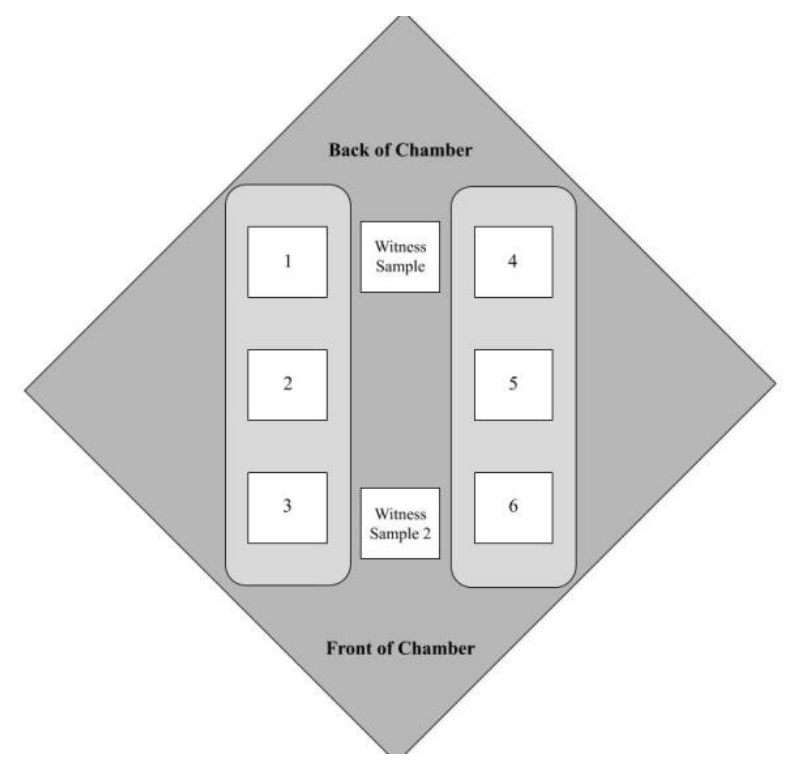

Figure 5.1: Location of Each Sample in the Trough Along with Witness Sample Location. 
Table 5.1: 24-Hour AO Test to Measure Varying Flux and Fluence for Each Sample Position.

\begin{tabular}{|c|c|c|c|c|}
\hline Test & Mass Loss (g) & $\begin{array}{c}\text { Flux } \\
\text { (atoms/cm2/s) }\end{array}$ & $\begin{array}{c}\text { Fluence } \\
\text { (atoms/cm2) }\end{array}$ & Position \\
\hline \multirow{8}{*}{1} & $0.0194 \pm 0.0010$ & $\begin{array}{c}9.909 \pm \\
1.23 \cdot 1015\end{array}$ & $\begin{array}{c}8.561 \pm \\
1.92 \cdot 10_{20}\end{array}$ & Witness Sample \\
\hline & $0.0254 \pm 0.0010$ & $\begin{array}{c}1.119 \pm \\
0.133 \cdot 1016\end{array}$ & $\begin{array}{c}9.671 \pm \\
1.15 \cdot 10_{20}\end{array}$ & 1 \\
\hline & $0.036 \pm 0.0009$ & $\begin{array}{c}1.675 \pm \\
0.193 \cdot 1016\end{array}$ & $\begin{array}{c}1.447 \pm \\
0.167 \cdot 10_{21}\end{array}$ & 2 \\
\hline & $0.0206 \pm 0.0005$ & $\begin{array}{c}9.323 \pm \\
1.05 \cdot 1015\end{array}$ & $\begin{array}{c}8.055 \pm \\
0.904 \cdot 10_{20}\end{array}$ & 3 \\
\hline & $0.0296 \pm 0.0009$ & $\begin{array}{c}1.333 \pm \\
0.159 \cdot 1016\end{array}$ & $\begin{array}{c}1.151 \pm \\
0.137 \cdot 10_{21}\end{array}$ & 4 \\
\hline & $0.0338 \pm 0.0008$ & $\begin{array}{c}1.544 \pm \\
0.177 \cdot 1016\end{array}$ & $\begin{array}{c}1.334 \pm \\
0.153 \cdot 10_{21}\end{array}$ & 5 \\
\hline & $0.023 \pm 0.0010$ & $\begin{array}{c}1.117 \pm \\
0.141 \cdot 1016\end{array}$ & $\begin{array}{c}9.647 \pm \\
1.22 \cdot 10_{20}\end{array}$ & 6 \\
\hline & $0.023 \pm 0.0011$ & $\begin{array}{c}1.324 \pm \\
0.168 \cdot 1016\end{array}$ & $\begin{array}{c}1.144 \pm \\
0.145 \cdot 10_{21}\end{array}$ & $\begin{array}{c}\text { Witness Sample } \\
2\end{array}$ \\
\hline
\end{tabular}

From the table, samples in positions 2 and 5 showed the highest flux and fluence for the 24-hour test. The samples ranged from 3.2-5.7 months of on orbit AO exposure in the ram direction of a $28.5^{\circ}$ inclined, $400 \mathrm{~km}$ altitude orbit. For every comparison to on-orbit duration in this thesis, the calculated time is for the ram direction of a spacecraft in a $28.5^{\circ}$ inclined, $400 \mathrm{~km}$ orbit [8]. The reason for the difference in flux and fluence for each Kapton sample comes from their location in the chamber. The trough plate consists of two parallel 
extruded sections that are closer to the RF electrode at their center than at their edges. Any samples that are placed in the middle of each trough will have higher AO flux and fluence than samples placed at the ends of the troughs since they are closer to the RF electrode. Kapton samples in positions 2 and 5 in table 5.1 were placed in the center of the troughs and experienced significantly more mass loss than the other samples. This variation was also observed in almost every test that used the trough plate to test antennas with the exception of test 3 where the sample in position 3 saw the greatest mass loss in the trough. A test was conducted earlier in the year by other students that measured an average fluence value of $1.58 \pm 0.05 \cdot 1016$ over multiple 24-hour exposure tests [54]. Compared to the samples placed in this test only the samples in positions 2 and 5 showed values that were similar to the values recorded by other students. The test conducted by the other students used a different plate and had witness samples positioned closer to the center of the chamber during testing than the witness samples in this thesis. Since the troughs in the plate are cut completely through the plate, aluminum tape needed to be used to create a surface to support the samples. The tape was not perfectly flat and there was concern that AO could potentially erode the underside of the Kapton during the test. To reduce this, the sides of the Kapton were pinned down with aluminum tape that had been folded so that the adhesive did not touch the sample. The setup can be seen in figure 5.2. 


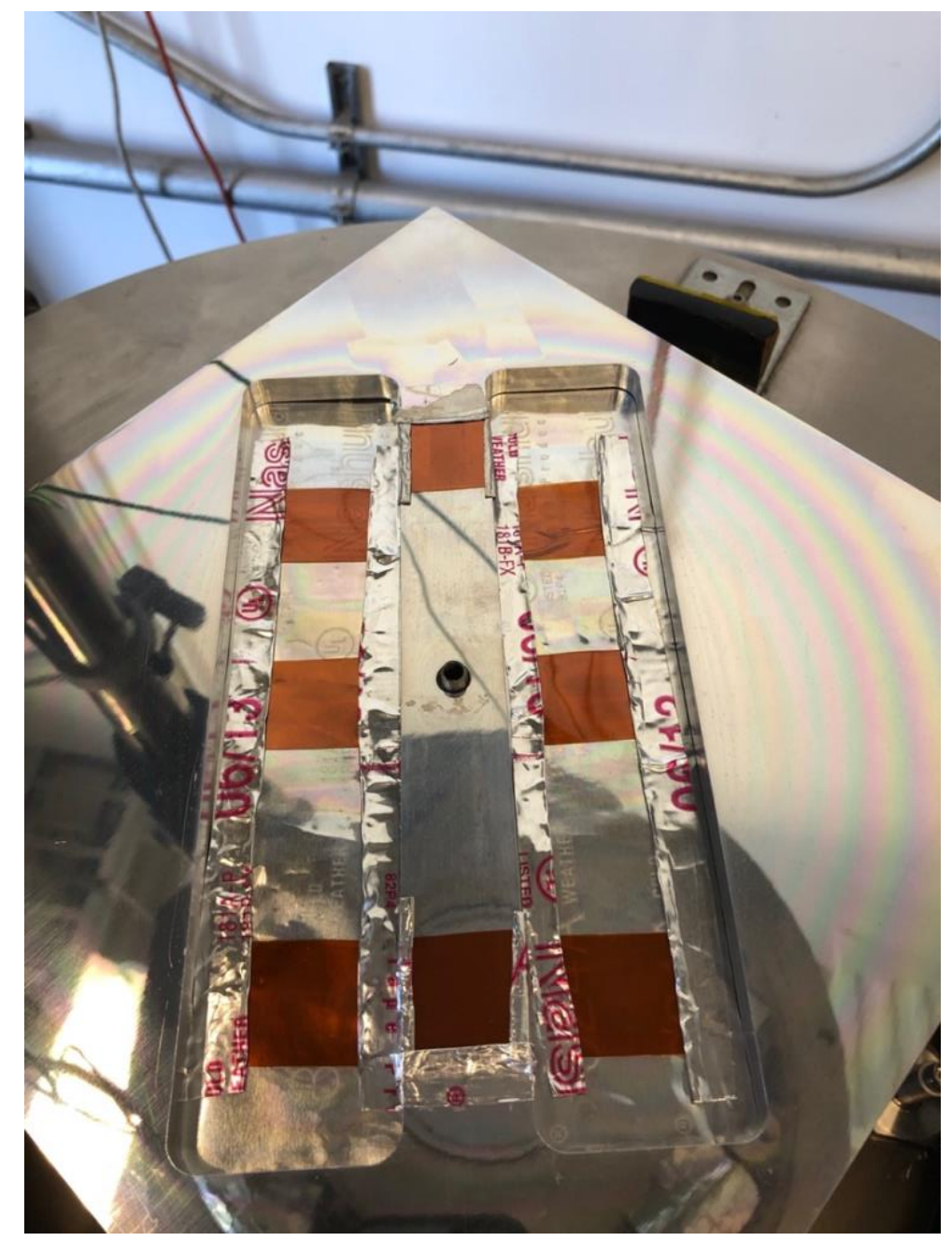

Figure 5.2: Kapton Sample Placement for 24-Hour Test to Measure AO Flux in Different Positions in the Chamber.

To determine the flux and fluence of these samples, the area needed to be calculated which was difficult to do by hand as the outline of the AO exposed area was not easy to measure by hand. To get a more accurate area measurement, an image processing software called ImageJ was used to measure the area of the AO eroded surface. The image processing software took an image and converted it to an 8-bit image and then to a binary image of white and black. During this conversion to a binary image, pixels on the edge of the eroded area were forced to become either white or black; so, to account for any error 
from the conversion to binary the calculated area had an error equal to plus or minus the number of pixels that make up the perimeter of the area times the pixel area. The pixel area was directly dependent on the distance between the Kapton and camera when the image was captured. The procedures for finding the area of the exposed portion of the Kapton sample using ImageJ can be found on the software's website [49]. It was known that there would be some error in the calculated area of the sample, so in order to compensate for this the perimeter of the calculated area was measured in pixels. For each image the exact area of each pixel was calculated and the error was calculated to be plus or minus the area of the pixels that made up the perimeter of the calculated area. The flux and fluence values for each AO test can be seen in table 5.2. This table shows how similar the flux values were between tests. The two most likely reasons the effective fluence varies in the 24-hour sample are error in the ImageJ software area calculation due to the border of the eroded area of the witness sample being indistinct, and small gaps between the witness sample and the tape allowing for small amounts of erosion under the tape. To compensate an additional $10 \%$ error in eroded area was included. This made it so all 24-hour and 48-hour tests had the average fluence of all same duration tests within the calculated error bars. 
Table 5.2: Comparison of the Witness Samples Flux and Fluence for Each AO Test Conducted.

\begin{tabular}{|c|c|c|c|c|}
\hline Test & Mass Loss (g) & $\begin{array}{c}\text { Flux } \\
\text { (atoms/cm2/s) }\end{array}$ & $\begin{array}{c}\text { Fluence } \\
\text { (atoms/cm2) }\end{array}$ & $\begin{array}{l}\text { Test Duration } \\
\text { (hours) }\end{array}$ \\
\hline 2 & $0.0174 \pm 0.0010$ & $\begin{array}{c}9.169 \pm \\
1.17 \cdot 1015\end{array}$ & $\begin{array}{c}7.922 \pm \\
1.01 \cdot 10_{20}\end{array}$ & 24 \\
\hline 3 & $0.0218 \pm 0.0007$ & $\begin{array}{c}1.133 \pm \\
0.129 \cdot 1016\end{array}$ & $\begin{array}{c}9.788 \pm \\
1.12 \cdot 10_{20}\end{array}$ & 24 \\
\hline 4 & $0.0380 \pm 0.0006$ & $\begin{array}{c}9.328 \pm \\
1.04 \cdot 1015\end{array}$ & $\begin{array}{c}1.612 \pm \\
0.180 \cdot 10_{21}\end{array}$ & 48 \\
\hline 5 & $0.0366 \pm 0.0010$ & $\begin{array}{c}9.131 \pm \\
1.05 \cdot 1015\end{array}$ & $\begin{array}{c}1.578 \pm \\
0.182 \cdot 10_{21}\end{array}$ & 48 \\
\hline 6 & $0.0194 \pm 0.0010$ & $\begin{array}{c}9.909 \pm \\
1.23 \cdot 1015\end{array}$ & $\begin{array}{c}8.561 \pm \\
1.92 \cdot 10_{20}\end{array}$ & 24 \\
\hline
\end{tabular}

\subsection{4-Hour Atomic Oxygen and Antenna Testing}

To establish whether there was a change in the patch antenna performance after exposure to AO, the antennas had to be tested in the anechoic chamber before and after exposure. The graphs below have the antennas prior to $\mathrm{AO}$ exposure represented by the solid lines and the antennas post $\mathrm{AO}$ exposure represented with stars. To make the charts easier to read, each chart only includes the patch antennas from the same AO test. Antennas 1-3 were not included in the analysis because they were tested on the original four sample plate rather than the trough plate and had their cables covered in aluminum tape. Table 5.3 shows the mass loss, flux, fluence, and position in the chamber of each antenna exposed for 24 hours. The flux and fluence values in the antenna mass loss tables were calculated from the witness sample of Kapton that was in the chamber for the duration of each test. 
These flux and fluence values were assumed to be the same for each antenna in the test.

For each test the witness sample was kept in the same location.

Table 5.3: 24-Hour Antenna Mass Loss Values and Flux and Fluence for Each Test

\begin{tabular}{|c|c|c|c|c|c|}
\hline Test & Antenna & $\begin{array}{l}\text { Mass Loss } \\
\text { (g) }\end{array}$ & $\begin{array}{c}\text { Flux } \\
\text { (atoms/cm2/s) }\end{array}$ & $\begin{array}{c}\text { Fluence } \\
(\text { atoms } / \mathrm{cm} 2)\end{array}$ & Position \\
\hline \multirow{6}{*}{2} & 4 & $\begin{array}{c}0.045 \pm \\
0.001\end{array}$ & \multirow{6}{*}{$\begin{array}{c}9.168 \pm \\
1.17 \cdot 1015\end{array}$} & \multirow{6}{*}{$\begin{array}{c}7.922 \pm \\
1.01 \cdot 10_{20}\end{array}$} & 4 \\
\hline & 5 & $\begin{array}{c}0.046 \pm \\
0.001\end{array}$ & & & 5 \\
\hline & 6 & $\begin{array}{c}0.033 \pm \\
0.001\end{array}$ & & & 1 \\
\hline & 7 & $\begin{array}{c}0.057 \pm \\
0.001\end{array}$ & & & 2 \\
\hline & 8 & $\begin{array}{c}0.038 \pm \\
0.001\end{array}$ & & & 3 \\
\hline & 9 & $\begin{array}{c}0.043 \pm \\
0.001\end{array}$ & & & 6 \\
\hline \multirow{6}{*}{3} & 10 & $\begin{array}{c}0.029 \pm \\
0.001\end{array}$ & \multirow{6}{*}{$\begin{array}{c}1.133 \pm \\
0.129 \cdot 1016\end{array}$} & \multirow{6}{*}{$\begin{array}{c}9.788 \pm \\
1.12 \cdot 10_{20}\end{array}$} & 4 \\
\hline & 11 & $\begin{array}{c}0.044 \pm \\
0.001\end{array}$ & & & 5 \\
\hline & 12 & $\begin{array}{c}0.034 \pm \\
0.001\end{array}$ & & & 1 \\
\hline & 13 & $\begin{array}{c}0.037 \pm \\
0.001\end{array}$ & & & 2 \\
\hline & 14 & $\begin{array}{c}0.045 \pm \\
0.001\end{array}$ & & & 3 \\
\hline & 15 & $\begin{array}{c}0.041 \pm \\
0.001\end{array}$ & & & 6 \\
\hline
\end{tabular}

From table 5.3 after 24 hours, the antennas had lost between $4-9 \%$ of their postoutgassing mass. Due to an oversight during the antenna preparation test 2 had a lower 
fluence yet higher mass loss as the antennas in this test did not have a Kapton backing during testing. The wax paper saw larger amounts of erosion resulting in a larger mass loss. From test 3 onward each antenna was checked for a Kapton backing before any testing was conducted. The observed mass loss for each antenna was also statistically different from one another. The middle two positions saw the greatest mass loss in their respective trough with the exception of antenna 14 which was placed in position 3 in test 3 . Antenna 14 saw the greatest mass loss out of any antenna for this test. The most probable cause of this variation in the trend is that the antenna was not properly positioned for the test and was accidently placed closer to the RF electrode in the center of the upper plate. This would cause the antenna to experience a greater flux and fluence of AO which would lead to higher mass loss values. After exposing the patch antennas to AO for 24 hours, a white powdery substance had formed on the surface that could easily be rubbed off when touched. The ease at which the powdery substance was rubbed off the antenna shows that the powder was either not bonded to the surface or very weakly bonded. The powdery material was unexpected as it was thought the surface of the antenna would completely erode without leaving any substances behind. It was expected that the surface material of the antenna would act like Kapton when exposed to AO by becoming rougher, but not easily removable by simply touching the sample. The green color seen on the printed words of the antenna had also faded to white after the test. The wire that was connected to the antenna exhibited varying levels of erosion depending on its location in the trough. The antennas placed in positions 1 and 4 displayed more erosion in the lower portion of the wire than in the upper portion which can be seen in figure 5.3. The antennas placed in positions 2 and 5 presented approximately equal erosion along the entire wire as shown in figure 5.4. Finally, the 
antennas placed in positions 3 and 6 showed erosion in the top portion of the wire significantly more than in the lower portion of the wire which is seen in figure 5.5.

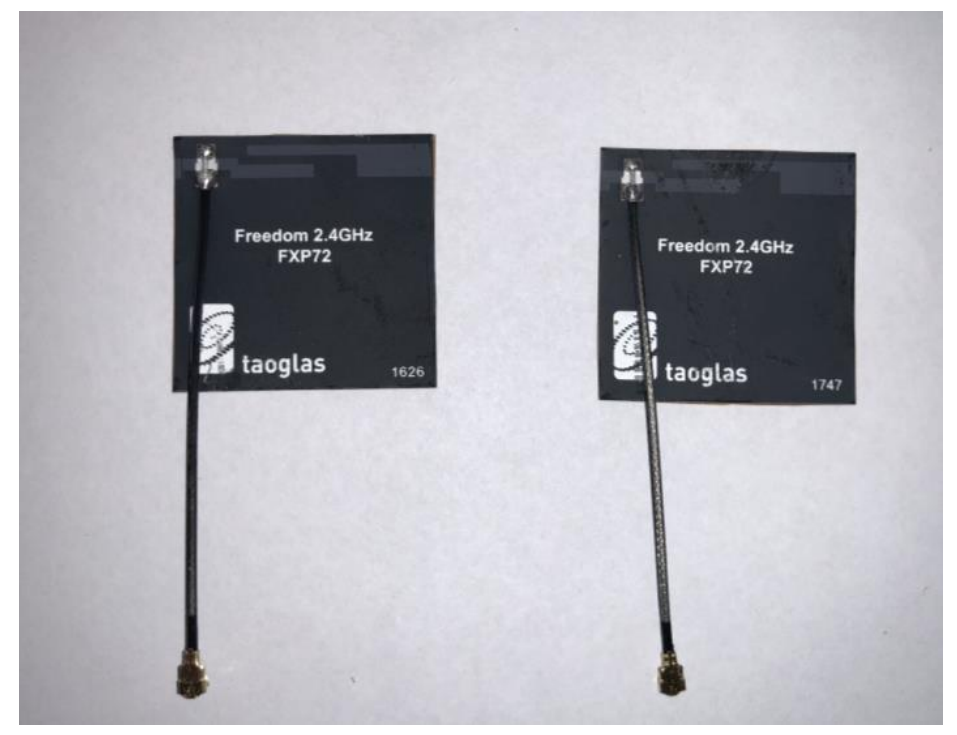

Figure 5.3: 24-Hour AO Exposed Samples that Were Placed in Positions 1 (Left) and 4 (Right).

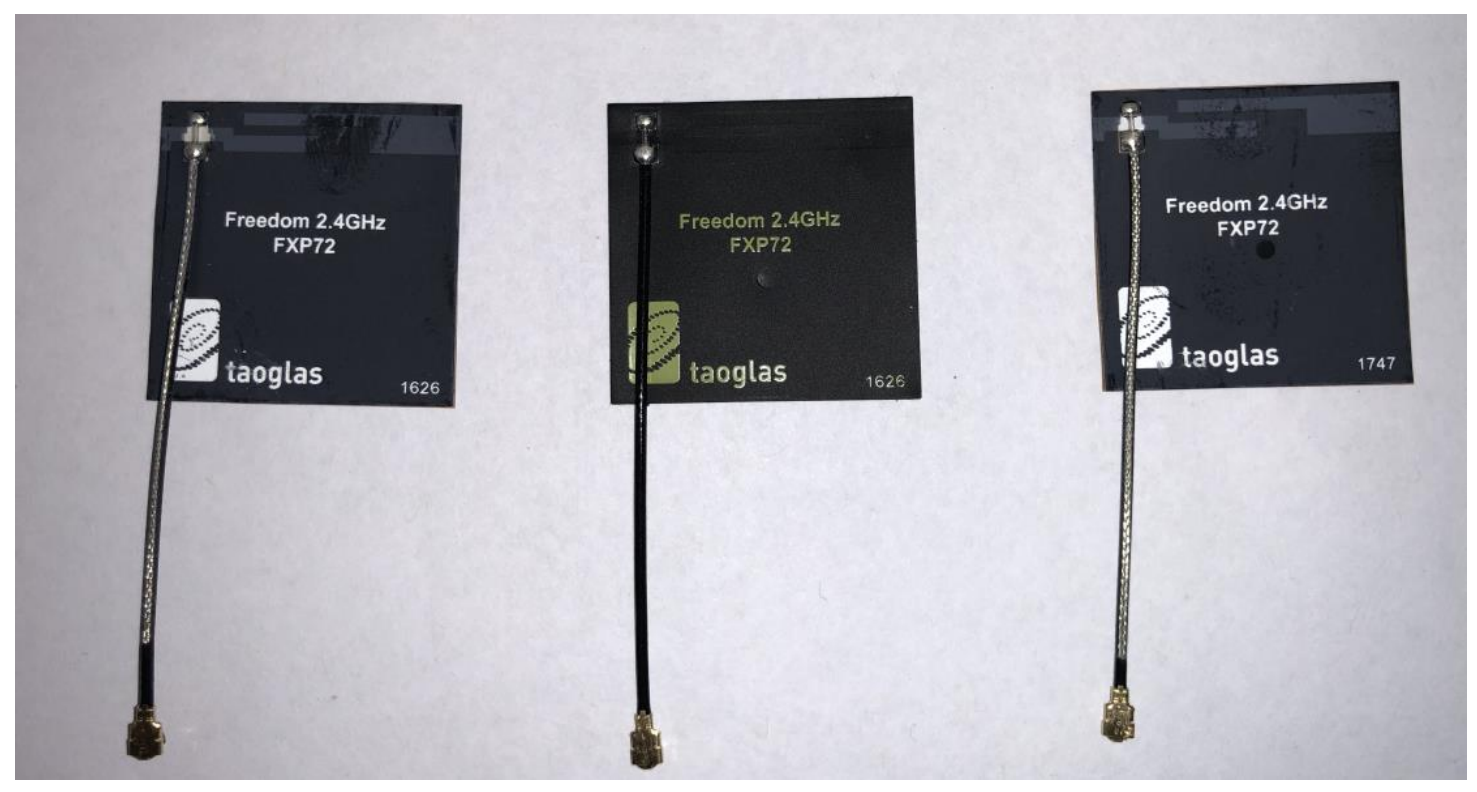

Figure 5.4: 24-Hour AO Exposed Samples that Were Placed in Positions 2 (Left) and 5 (Right) with an Untested Sample (Middle). 


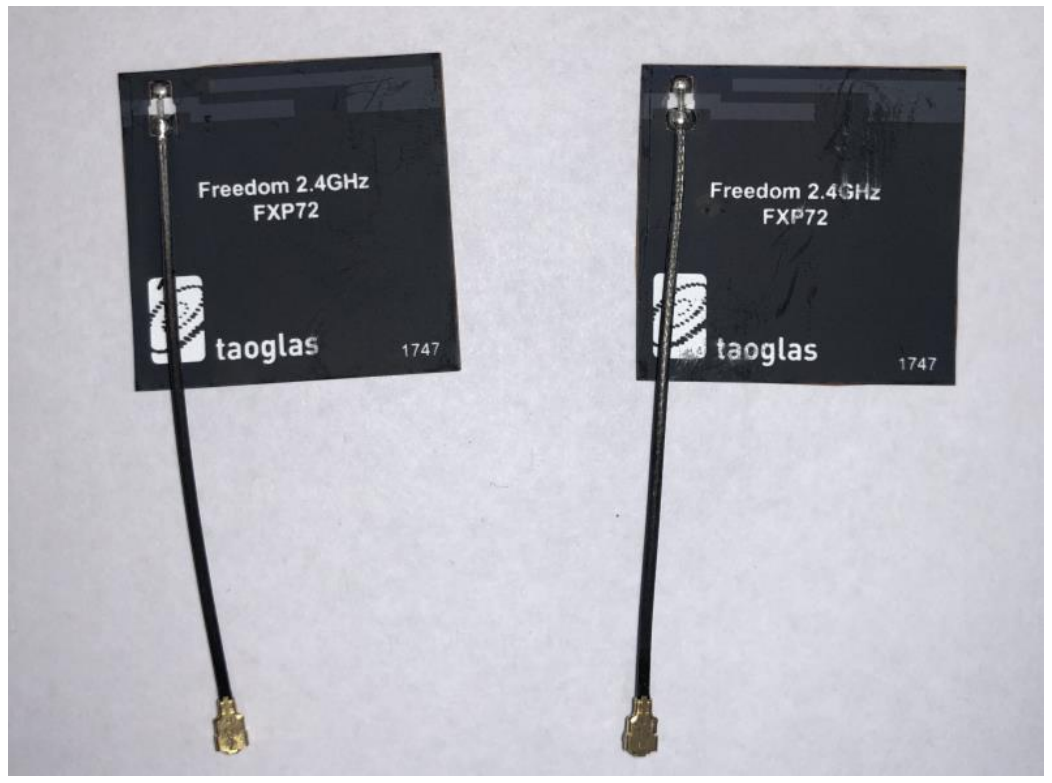

\section{Figure 5.5: 24-Hour AO Exposed Samples that Were Placed in Positions 3 (Left) and 6 (Right).}

After testing the antenna post $\mathrm{AO}$ exposure in the anechoic chamber the performance for each could be compared. Unfortunately, during the second round of performance testing it was observed that after each antenna was switched in the test setup, the gain of the antenna would drop further and further. It was determined that a new SMA cable was needed to connect the AUT to the VNA. After the new cable was installed, the problem did not reappear; however, because it was unknown when the cable first became erroneous, the data for the antennas that used the old cable could not be used for comparison. Between tests the antennas were stored in sealed Tupperware containers with their humidity levels monitored. Instead of completely starting over, the antennas that had already been exposed to $\mathrm{AO}$ were retested in the anechoic chamber with the new cable. The performance results from these tests were then compared to control antennas to see if there were any obvious differences in boresight gain. The boresight gain of an antenna is the axis of maximum gain for a directional antenna. For this patch antenna, the boresight gain happened to be normal 
to the surface of the patch antenna. This gain was measured to see if AO exposure affected the maximum gain or power the antenna could produce. A change in the boresight gain would mean a change in the maximum achievable data rate for the antenna. Figure 5.6 shows the change in gain of the 24-hour AO exposed patch antennas for after exposure.

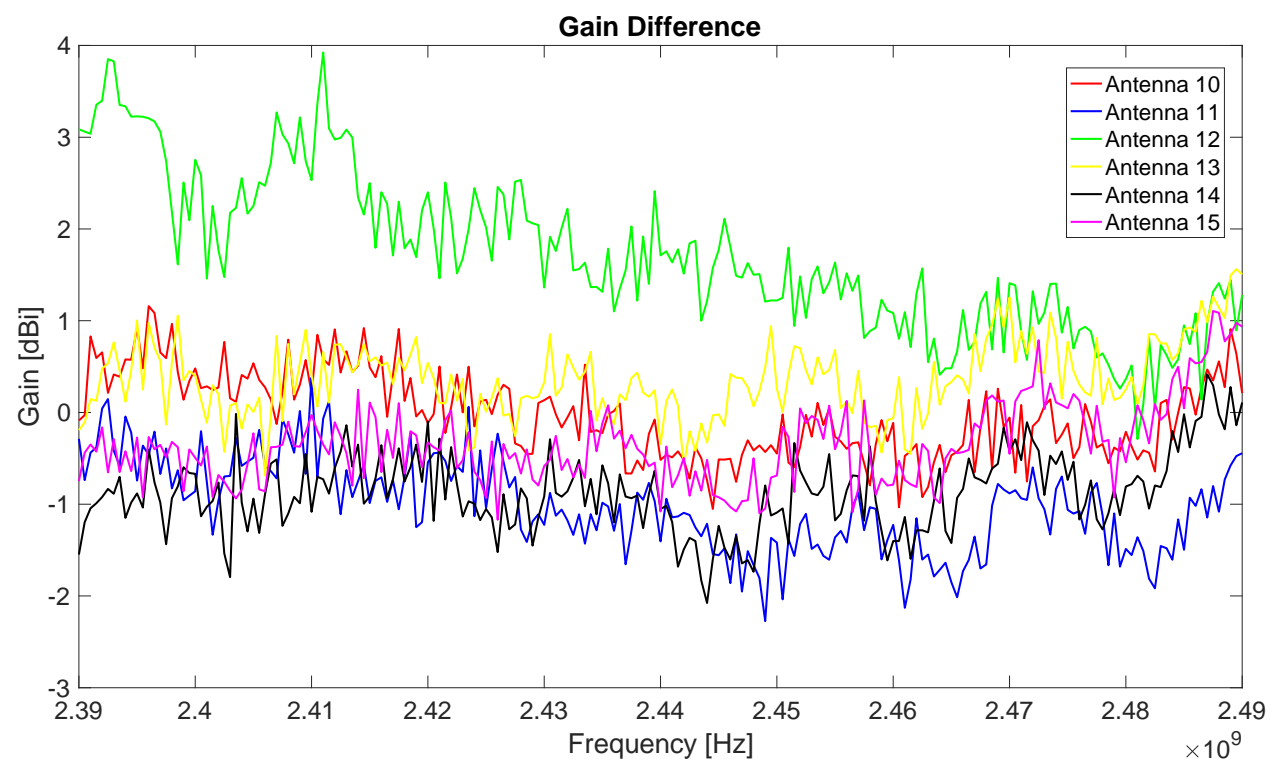

\section{Figure 5.6: Antennas 10-15 Change in Gain After 24-Hour AO Exposure.}

Since there was no apparent difference in the compared performance of the 24-hour exposed antennas and it did not appear as though AO was eroding through the outer layer of the antenna, it was decided that the antennas should be exposed to a longer, 48 -hour AO test. If the erosion rate of the surface of the patch antenna were to continue at the same rate, a longer AO test would degrade more of the outer coating, which was presumed to affect the antenna performance.

\subsection{8-Hour Atomic Oxygen and Antenna Testing}

Since the 48-hour AO test was double the original test time, there were concerns that the base plate could heat to a point that would exceed the patch antenna's upper survival 
temperature limit of $85^{\circ} \mathrm{C}$. To monitor this, thermocouples were attached to the top and bottom of the plate and measured after 24 hours, and every 2 hours after, for a duration of 6 hours, and then again before turning off the RF power. The plate reached a maximum temperature of $74.3^{\circ} \mathrm{C}$ after 28 hours and was never recorded exceeding that temperature. Table 5.4 shows the mass loss, flux, fluence, and position in the chamber of each antenna exposed for 48 hours.

Table 5.4: 48-Hour Antenna Mass Loss and Flux and Fluence for Each Test

\begin{tabular}{|c|c|c|c|c|c|}
\hline Test & Antenna & $\begin{array}{l}\text { Mass Loss } \\
\text { (g) }\end{array}$ & $\begin{array}{c}\text { Flux } \\
\text { (atoms/cm2/s) }\end{array}$ & $\begin{array}{c}\text { Fluence } \\
\text { (atoms/cm2) }\end{array}$ & Position \\
\hline \multirow{6}{*}{4} & 16 & $\begin{array}{c}0.047 \pm \\
0.001\end{array}$ & \multirow{6}{*}{$\begin{array}{c}9.328 \pm \\
1.04 \cdot 1015\end{array}$} & \multirow{6}{*}{$\begin{array}{c}1.612 \pm \\
0.180 \cdot 10_{21}\end{array}$} & 1 \\
\hline & 17 & $\begin{array}{c}0.059 \pm \\
0.001\end{array}$ & & & 2 \\
\hline & 18 & $\begin{array}{c}0.048 \pm \\
0.001\end{array}$ & & & 3 \\
\hline & 19 & $\begin{array}{c}0.048 \pm \\
0.001\end{array}$ & & & 4 \\
\hline & 20 & $\begin{array}{c}0.060 \pm \\
0.001\end{array}$ & & & 5 \\
\hline & 21 & $\begin{array}{c}0.050 \pm \\
0.001\end{array}$ & & & 6 \\
\hline \multirow{5}{*}{5} & 22 & $\begin{array}{c}0.048 \pm \\
0.001\end{array}$ & \multirow{5}{*}{$\begin{array}{l}9.131 \pm \\
1.05 \cdot 1015\end{array}$} & \multirow{5}{*}{$\begin{array}{c}1.578 \pm \\
0.182 \cdot 10_{21}\end{array}$} & 1 \\
\hline & 23 & $\begin{array}{c}0.058 \pm \\
0.001\end{array}$ & & & 2 \\
\hline & 24 & $\begin{array}{c}0.054 \pm \\
0.001\end{array}$ & & & 3 \\
\hline & 25 & $\begin{array}{c}0.057 \pm \\
0.001\end{array}$ & & & 4 \\
\hline & 26 & $\begin{array}{c}0.066 \pm \\
0.001\end{array}$ & & & 5 \\
\hline
\end{tabular}


For the 48-hour test, it was observed that the antennas lost an average of 0.00854 grams more mass than the 24-hour tests. The effective fluence also showed an on-orbit time of approximately 6.4 months for the previously specified orbit and orientation. Even though the antenna were exposed for double the time the mass loss did not double for the antenna. It was suspected that the white powder left behind might be affecting the mass loss by reducing the erosion rate on the antennas. Position 6 was not used in test 5 as there were not enough antennas available. The mass loss in these tests were again statistically different than each other. For both test 4 and 5 the sample in position 2 and 5 had the highest mass loss in its trough. This result supports the data collected from the MAX verification test as the samples in position 2 and 5 from that test had the two highest calculated fluences in their rough. In addition to the added mass loss, the wire attached to the antenna was significantly more eroded for every position in the chamber although the same trend in wire erosion location occurred. A new characteristic that was seen in the antennas exposed for 48 hours that was not seen in the antennas that were exposed for 24 hours was a slight gradient in the color of the powder that coated the surface. The powder started as one color in the center of the patch antenna and gradually changed to different colors as the powder got closer to the outside edge of the patch antenna. Figures 5.7, 5.8, and 5.9 show the samples after a 48 hour AO test and their positions in the trough. 


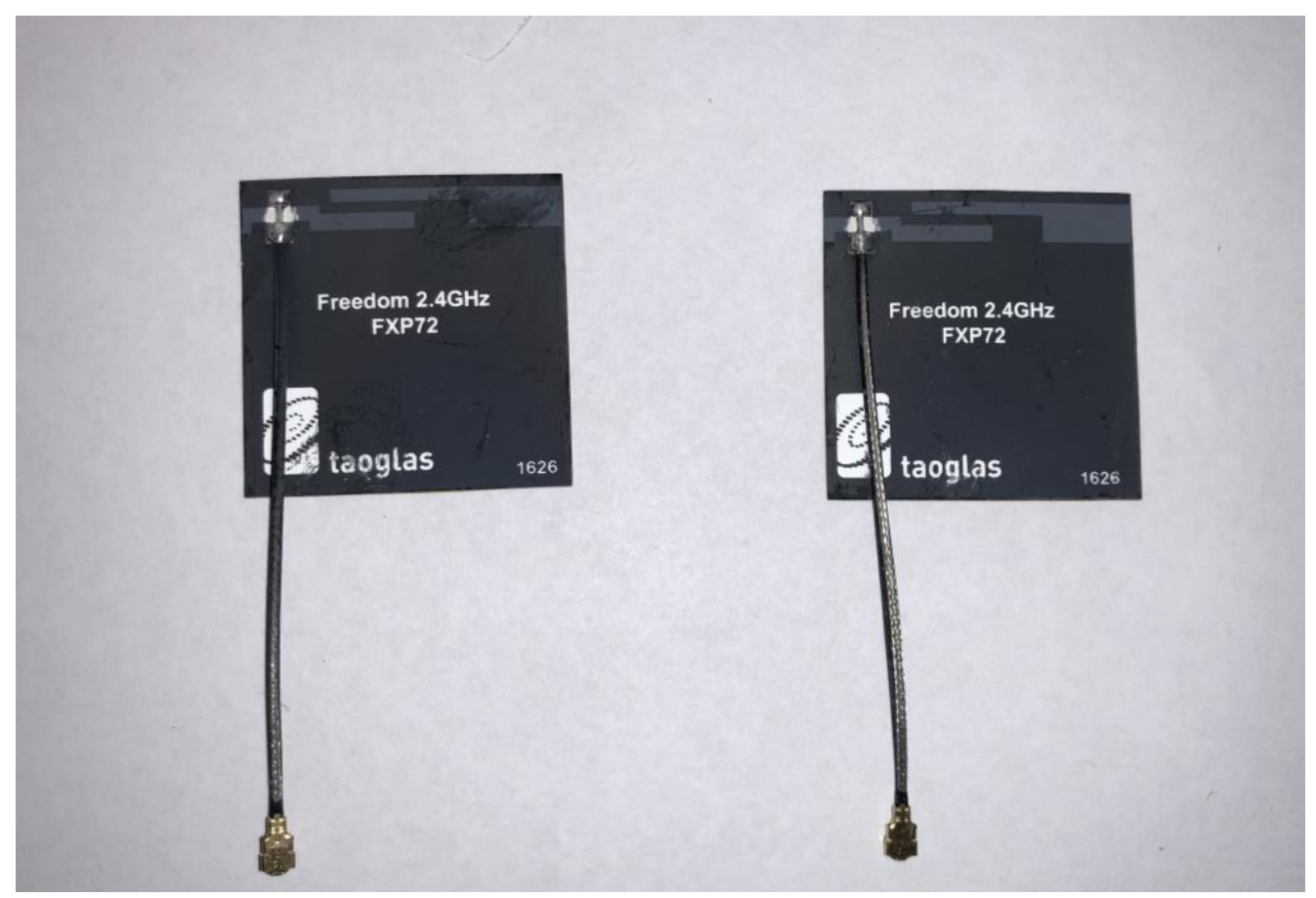

Figure 5.7: 48-Hour AO Exposed Samples that Were Placed in Positions 1 (Left) and 4 (Right).

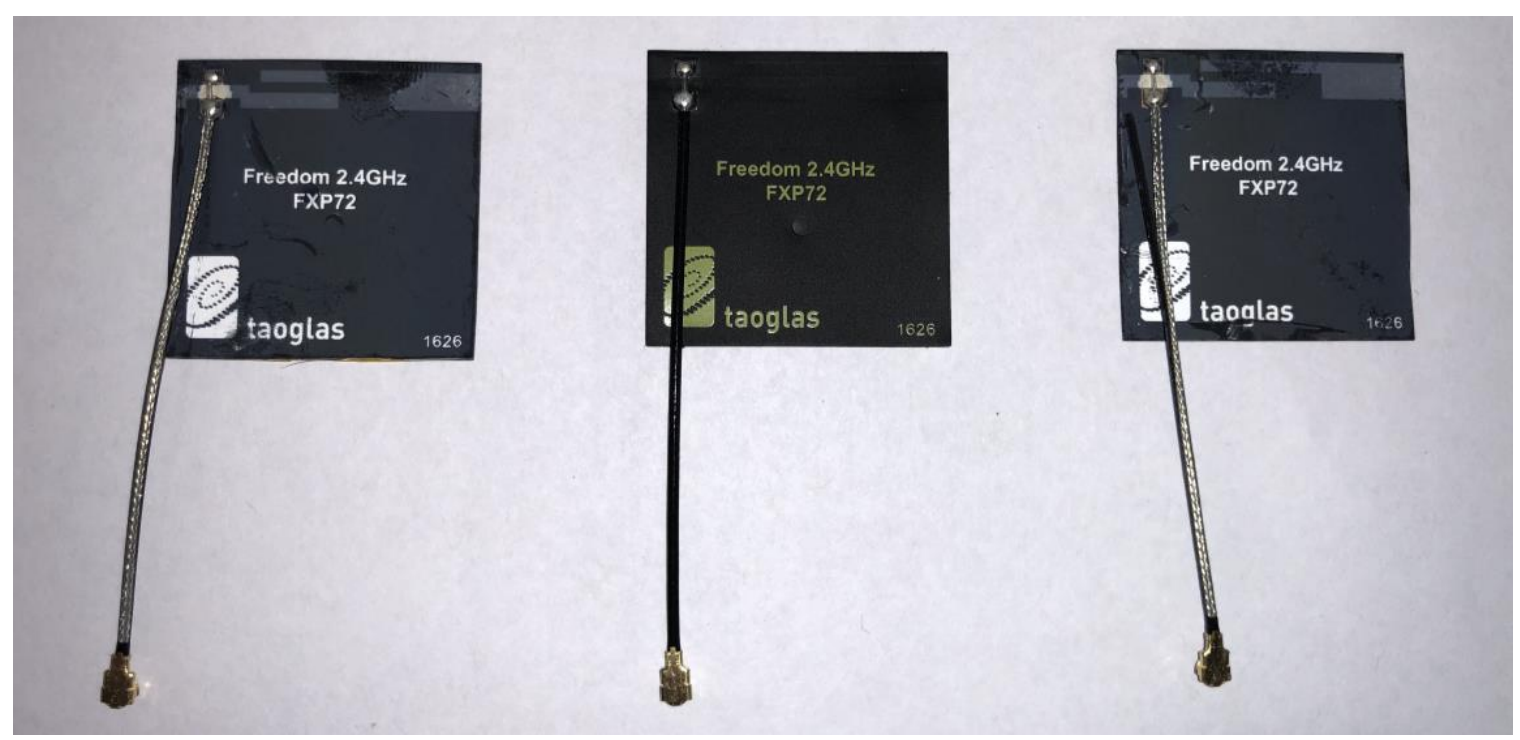

Figure 5.8: 48-Hour AO Exposed Samples that Were Placed in Positions 2 (Left) and 5 (Right) with an Untested Sample (Middle). 


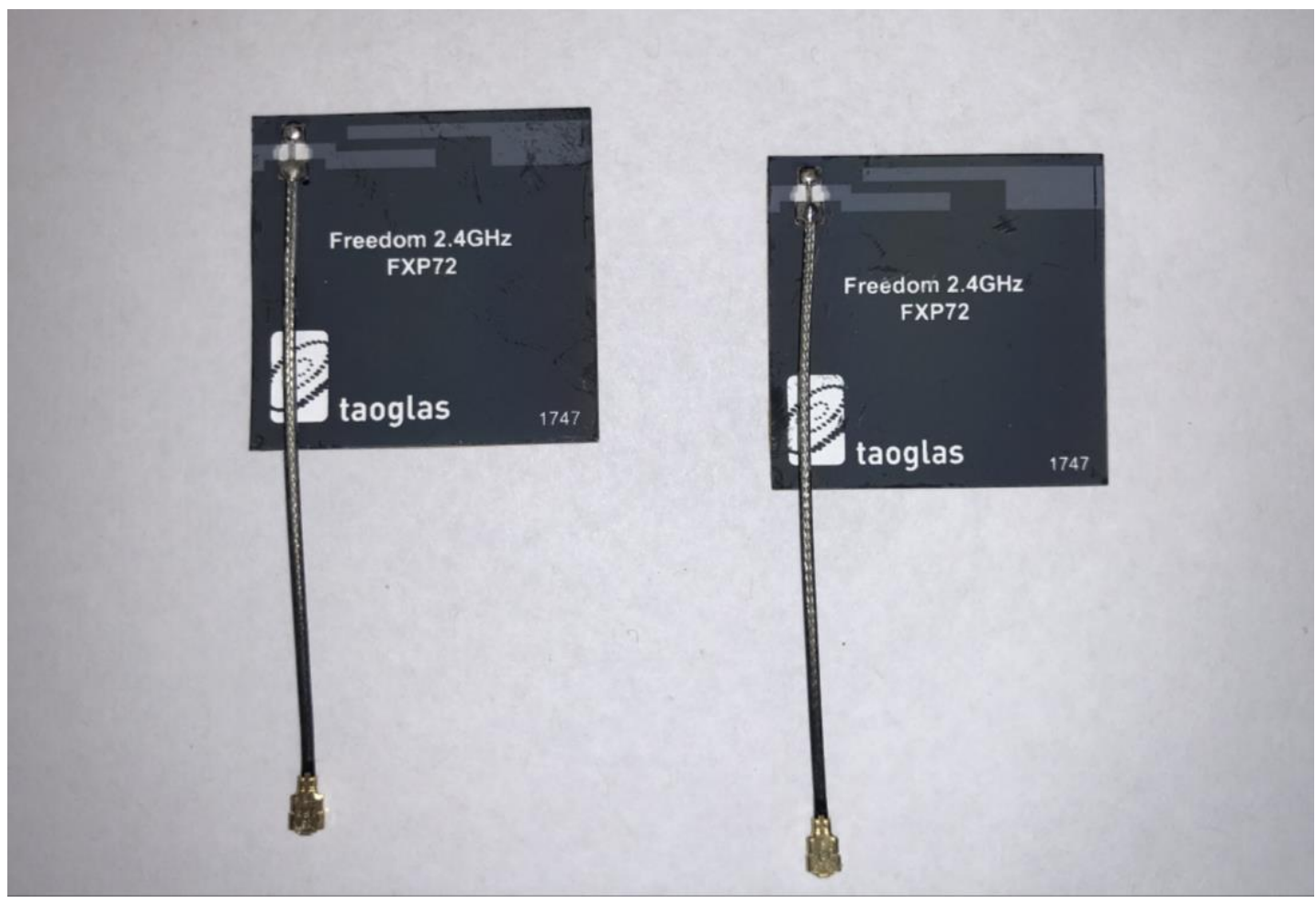

Figure 5.9: 48-Hour AO Exposed Samples that Were Placed in Positions 3 (Left) and 6 (Right).

To determine if there was a statistically significant difference in the patch antenna performance before and after exposure to $\mathrm{AO}$, a paired samples t-test was used to compare the antenna boresight gain. A t-test compares the means of two groups and tells you whether they are different from each other [55]. From the test, the data will result in a pvalue, which is the probability that the results from your sample occurred by random chance. As an example, a p-value of 0.05 means there is a $5 \%$ chance that the results occurred due to random chance. For most cases a p-value of 0.05 or lower is the standard for accepting that the data is valid [55]. This test was used to compare the gain over the entire frequency range as well as comparing just the lower half and just the upper half of the frequency range. The entire frequency range was compared to look for an overall drop in antenna performance, while the two halves were each tested to see if AO exposure would 
affect lower or higher frequencies more. When conducting a statistical test, the null hypothesis is the result that the researcher would expect to see if the test does not make a change in the expected outcome, while the alternative hypothesis is what will happen if the test does make a change in the expected outcome. For this statistical test, the null hypothesis stated that the average gain of the patch antenna before AO exposure would be equal to the average gain of the patch antenna after AO exposure. Figures 5.10 and 5.11 show the change in antenna performance for the boresight gain over the frequency range 2.39-2.49 GHz for the antennas exposed for 48 hours. The varied frequency was analyzed to see if the $\mathrm{AO}$ exposure would affect the antenna performance at different frequencies more than others.

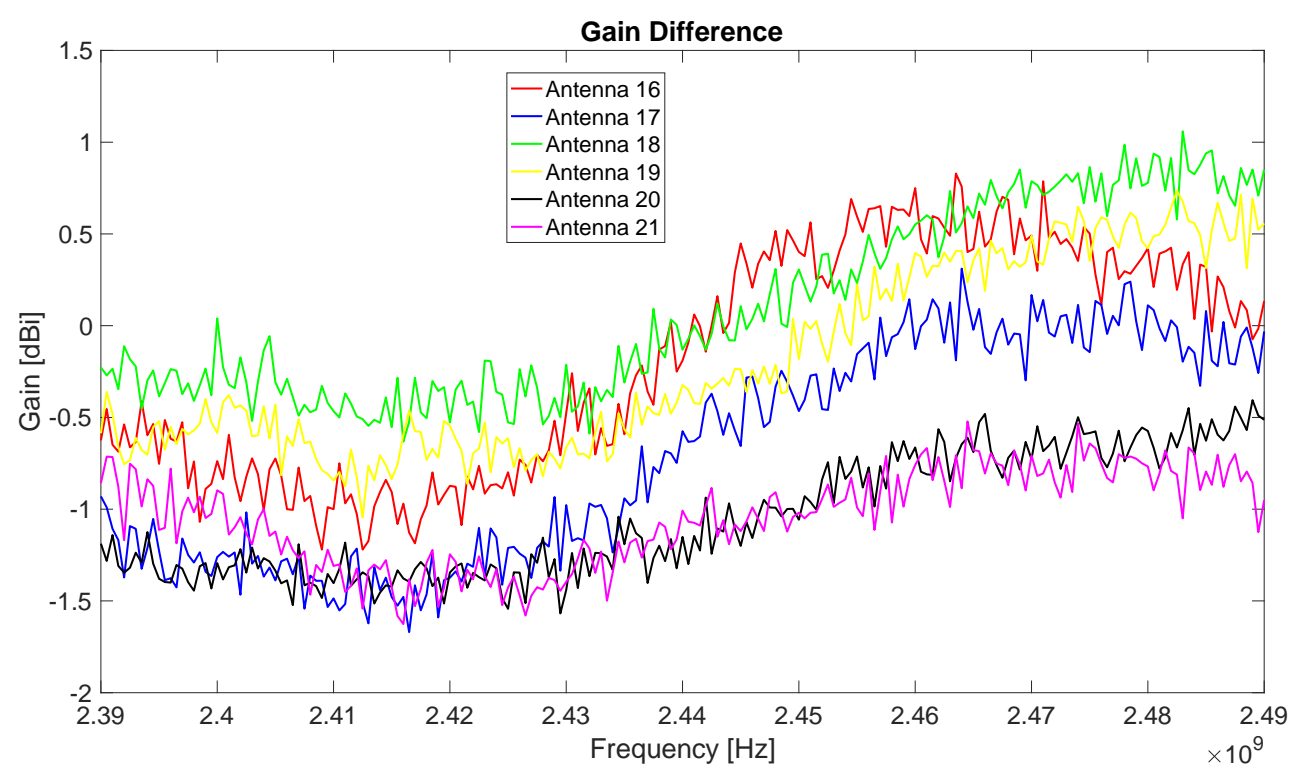

Figure 5.10: Antennas 16-21 Change in Gain After 48-Hour AO Exposure. 


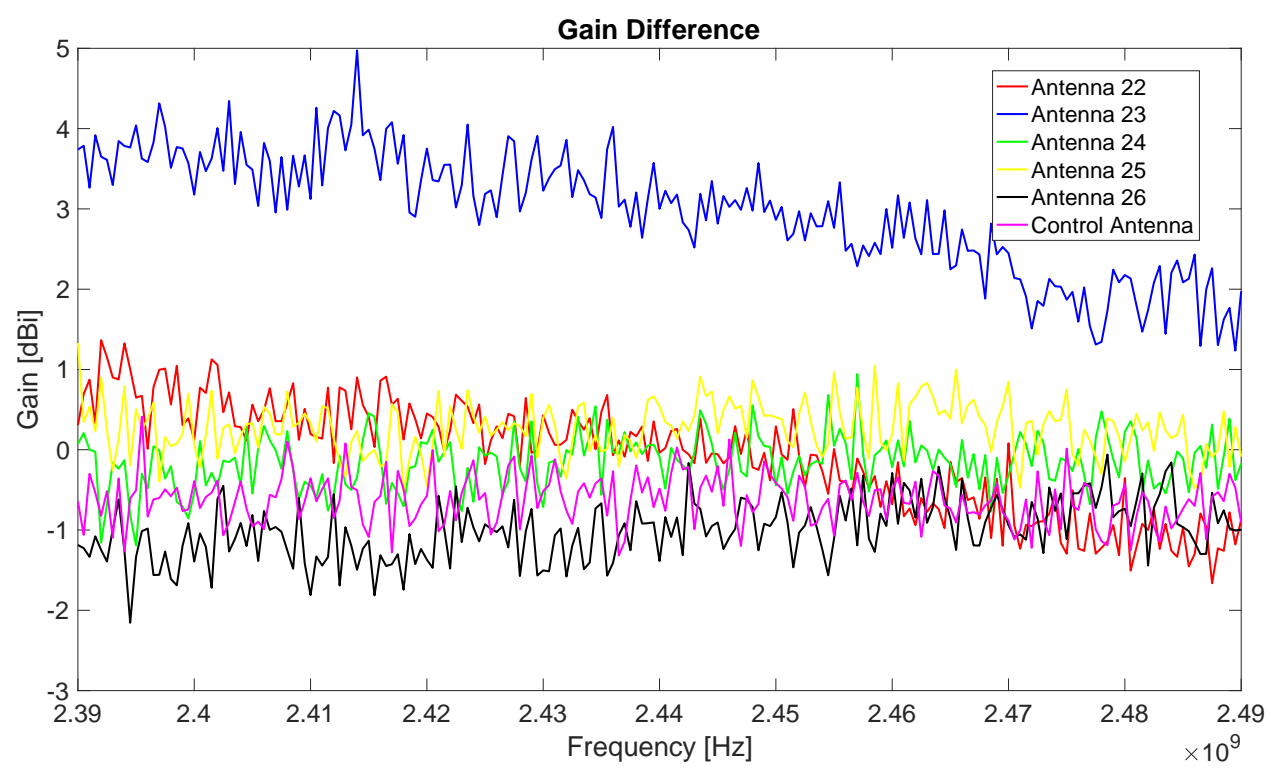

Figure 5.11: Antennas 22-26 Change in Gain After 48-Hour AO Exposure.

The antennas from the first 48-hour test shown in figure 5.10 showed a slight decline in performance of the antennas after exposure to AO; however, when a second test was run the trend was not observed again. Even though each position had different amounts of mass loss there did not appear to be a consistent trend in mass loss versus performance. The variance between the measurements for each antenna was found to be $0.677 \mathrm{dBi}$. For the findings of a test to be valid, the results must be repeatable. A third test was not run since there was no apparent change in the antenna performance, so the scope of the thesis shifted to focus on how the $\mathrm{AO}$ exposure was affecting the surface material. Additionally, the results matched those seen from the 24-hour samples. The results from the paired samples $\mathrm{t}$-test for the 48-hour samples can be seen in table 5.5. The reason there was a negative gain is due to the losses in the system itself. Additional losses in the anechoic chamber will cause the overall gain to be lower than the true gain. By connecting a short cable directly from port 1 to port 2 it was possible to measure the losses in the system due to the SMA cables. When the long SMA cables used during testing were connected to each other via a 
barrel adapter, the system measured an $\mathrm{S}_{21}$ of $-13.7 \mathrm{~dB}$. When port 1 was directly connected to port 2 via a short SMA cable an $\mathrm{S}_{21}$ of $-3.7 \mathrm{~dB}$ was measured meaning the long cables added a loss of $10 \mathrm{~dB}$ to the system. For the SMA to U.FL adapter a loss of $0.57 \mathrm{~dB}$ was measured when directly connecting the two ports of the VNA.

\section{Table 5.5: Paired Samples T-Test for 48-Hour Exposed Patch Antenna Gain Over Various Frequencies.}

\begin{tabular}{cccc}
\hline $\begin{array}{c}\text { Frequency Range } \\
(\mathrm{GHz})\end{array}$ & $\begin{array}{c}\text { Pre-Exposure Mean } \\
\text { Gain }(\mathrm{dBi})\end{array}$ & $\begin{array}{c}\text { Post-Exposure } \\
\text { Mean Gain }(\mathrm{dBi})\end{array}$ & P-Value \\
\hline $2.3900-2.4395$ & -6.219 & -6.255 & 0.940 \\
$2.4400-2.4900$ & -6.047 & -5.756 & 0.338 \\
$2.3900-2.4900$ & -6.132 & -6.004 & 0.739 \\
\hline
\end{tabular}

In order for the test to be significant, the p-value must be below 0.10 to demonstrate some statistical significance and below 0.05 to show strong statistical significance. A pvalue of 0.05 means there is at least a $95 \%$ chance that the null hypothesis is false and can be rejected. For all the tests run, there was not a statistically significant result and so it was not possible to reject the null hypothesis, meaning the results of the test did not show a statistically significant change in the boresight gain.

The radiation patterns for the antenna were also measured both before and after $\mathrm{AO}$ exposure. To identify differences in the radiation pattern, the gain was examined for values that exceeded the normal variability between tests. From looking at the plots of the E-plane and H-plane of the antennas, no apparent difference could be seen in the radiation pattern shape or gain. 
All the radiation patterns were within a normal range of variability except for antenna 16 in the E-plane in figure 5.12. However, because antenna 16 is the only antenna to be different, and is only different in the E-plane, not the H-plane, it was decided that this result was likely erroneous as no other antenna experienced a similar change. The most likely cause for this result could be from the cable connection between the VNA, and the antenna becoming snagged while the positioner was moving to measure the radiation pattern. This would cause the antenna to bend in its mount which would result in erroneous data being measured. The radiation pattern was measured at $2.44 \mathrm{GHz}$ for each test and was rotated $360^{\circ}$ about both its E-plane and its H-plane. The figures 5.12, 5.13, 5.14, and 5.15 show the patch antenna radiation patterns with the solid lines representing the antennas before $\mathrm{AO}$ exposure and the dotted lines representing the antennas after the $\mathrm{AO}$ exposure. If there was to be a difference, the expected change would be seen as a change in size of the lobes in each of the planes, as that would represent a change in gain.

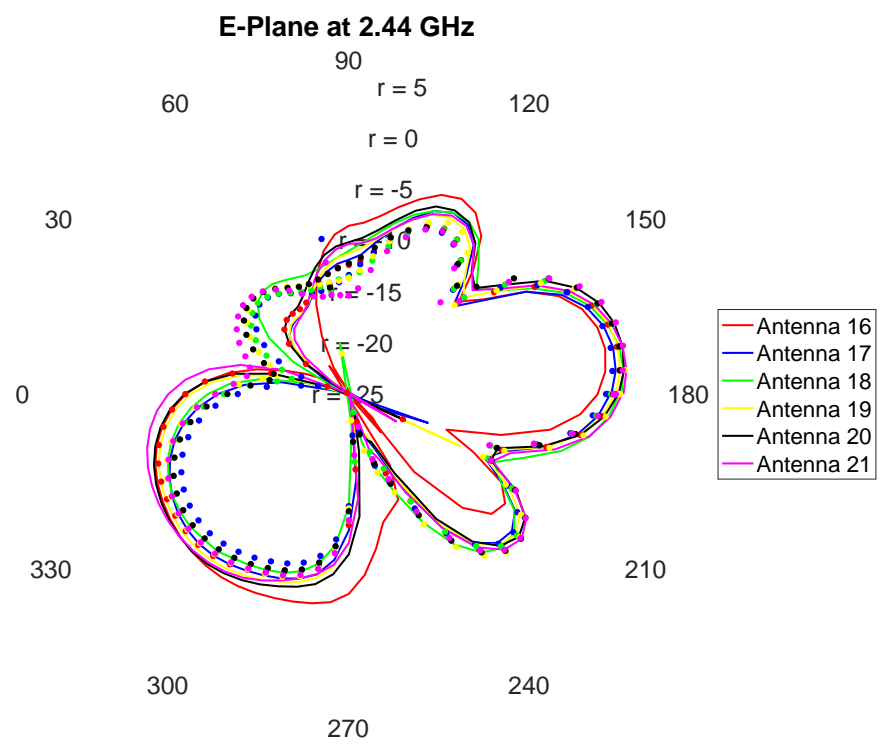

Figure 5.12: Antennas 16-21 Radiation Pattern in the E-Plane Measured Before (Solid Lines) and After (Dotted Lines) 48 Hours of AO Exposure. 


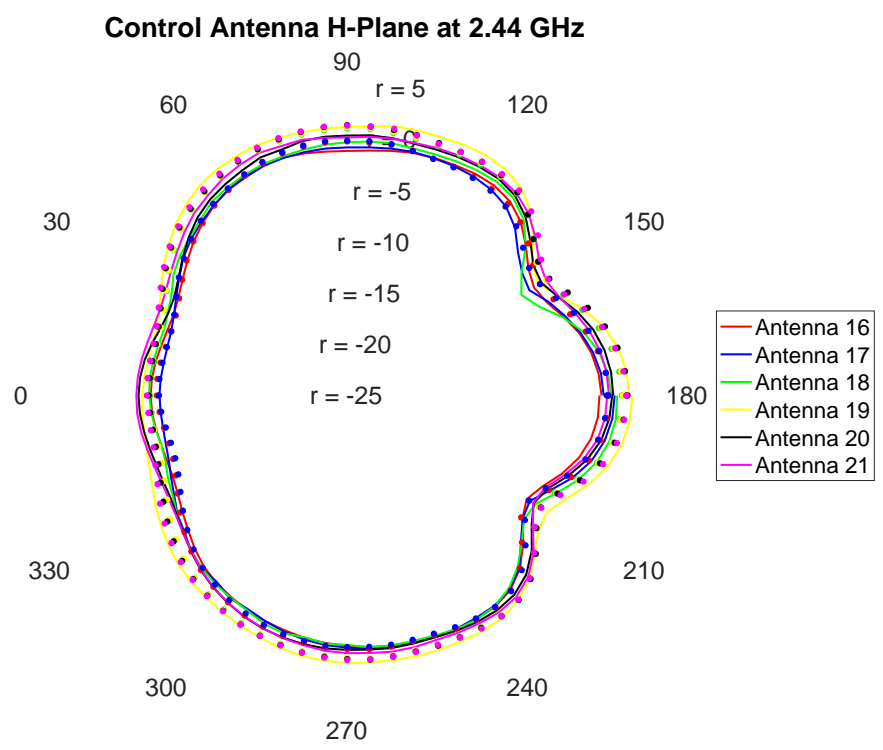

Figure 5.13: Antennas 16-21 Radiation Pattern in the H-Plane Measured Before (Solid Lines) and After (Dotted Lines) 48 Hours of AO Exposure.

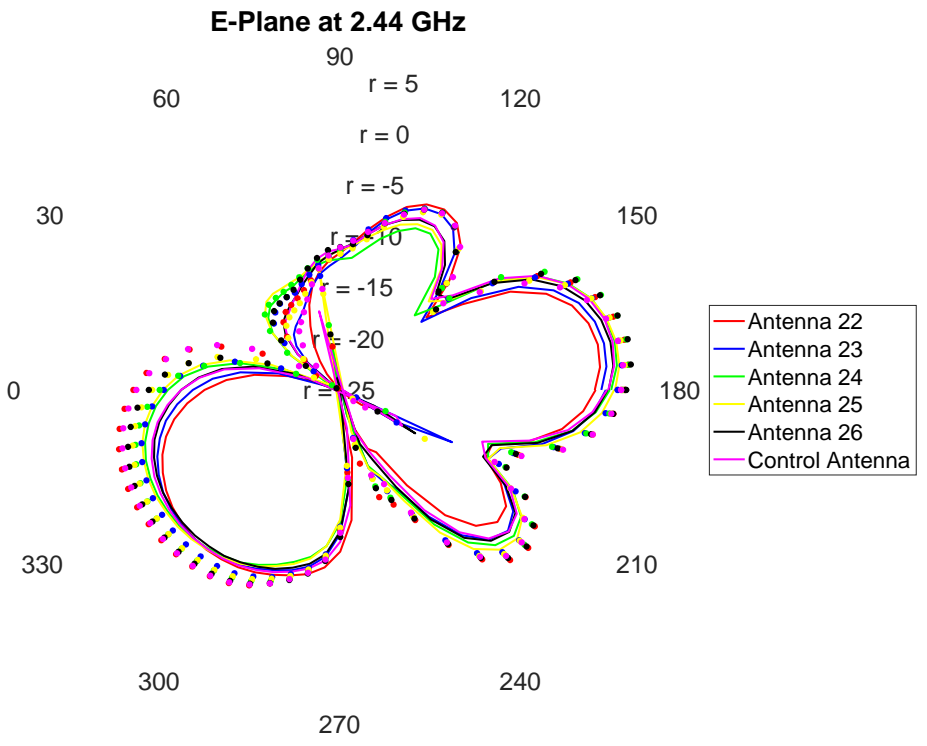

Figure 5.14: Antennas 22-26 Radiation Pattern in the E-Plane Measured Before (Solid Lines) and After (Dotted Lines) 48 Hours of AO Exposure. 


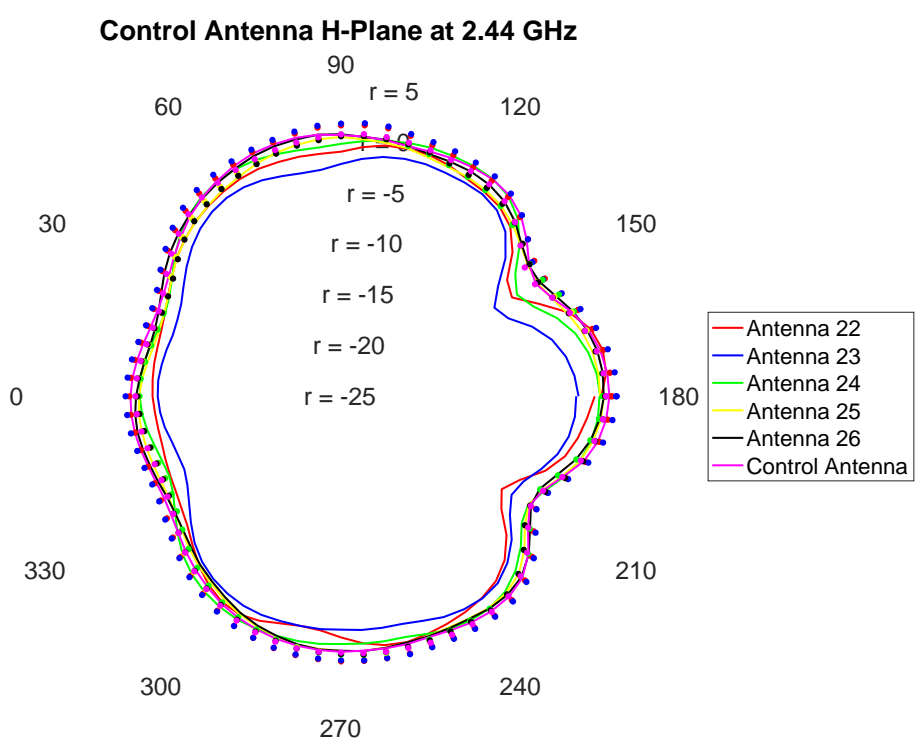

Figure 5.15: Antennas 22-26 Radiation Pattern in the H-Plane Measured Before (Solid Lines) and After (Dotted Lines) 48 Hours of AO Exposure.

The measured radiation pattern did not match up with the radiation pattern reported in the product data sheet. The antenna measurements from the data sheet used ABS plastic to act as the dielectric substrate and no ground plane [38]. The antenna tested in this thesis had a different backing of Plexiglas used as the dielectric substrate and no ground plane. ABS plastic has a dielectric constant of 2.7 while Plexiglas has a dielectric of 3.4 [56]. Other studies have shown that different dielectric constants will result in a different radiation pattern for the material [57][58]. A previous a study comparing the affect dielectric materials has on antenna characteristics showed that by changing the dielectric permittivity of the antenna material by only 0.4 can cause new lobes and null points to form [59]. Different lobes and null points were also seen when comparing the measured radiation pattern to the data sheets where the difference in the dielectric was 0.7 . The same study showed a permittivity change from 3.66 to 4.4 caused the size of the back lobe to drop by about $6 \mathrm{dBi}$ [59]. This change of $6 \mathrm{dBi}$ is similar to two of the measured lobes in the Eplane when compared to the E-plane in the data sheet. To be sure that the measured 
radiation pattern was not an error the antenna was flipped in the mount and measured again. The measurement found that the antenna was still radiating stronger out of the back of the antenna since there was no ground plane to reflect the signal.

A ground plane was not used during this thesis with AO exposed samples since the data sheet had measurements for samples without a ground plane and the ground plane would not be exposed to $\mathrm{AO}$ as it is underneath the patch antenna when operating. In the data sheet, several of the provided measurements are for an antenna with a ground plane but the specific material of the ground plane is not specified. To compare the antenna performance with and without a ground plane a copper ground plane measuring $15.24 \mathrm{~cm}$ by $8.89 \mathrm{~cm}$ was mounted to an antenna per the manufacturer specifications. The gain and radiation pattern were compared to the control antenna used during the $\mathrm{AO}$ exposure testing. Figure 5.16 shows the antenna gain while figures 5.17 and 5.18 show the antenna radiation pattern in the E-plane and H-plane respectively.

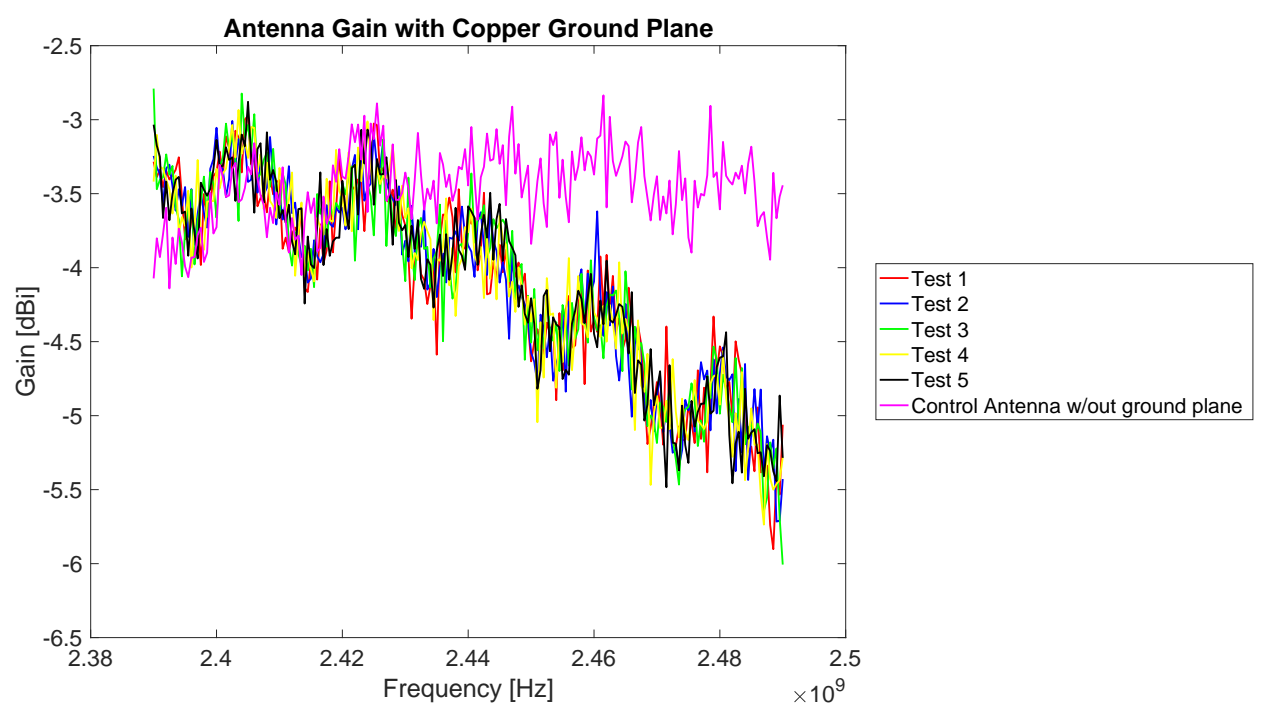

Figure 5.16: Comparison of Patch Antenna Gain with and without a Copper Ground Plane. 


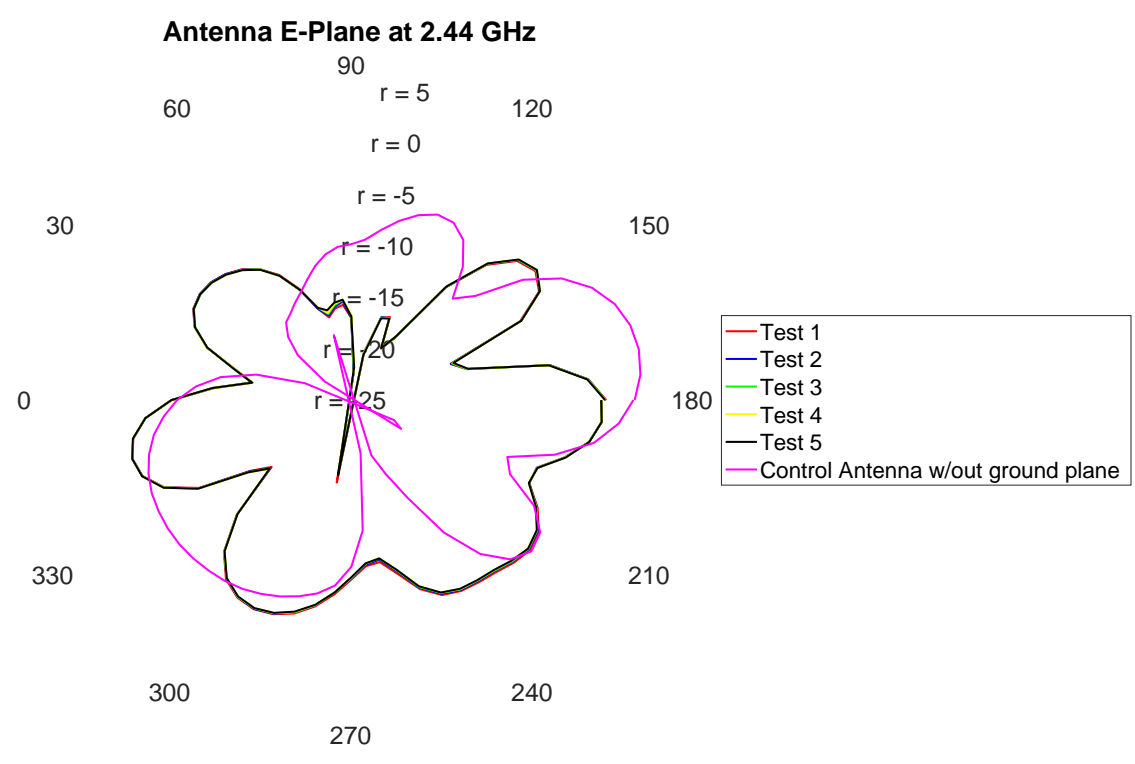

Figure 5.17: Comparison of Patch Antenna E-plane with and without a Copper Ground Plane.

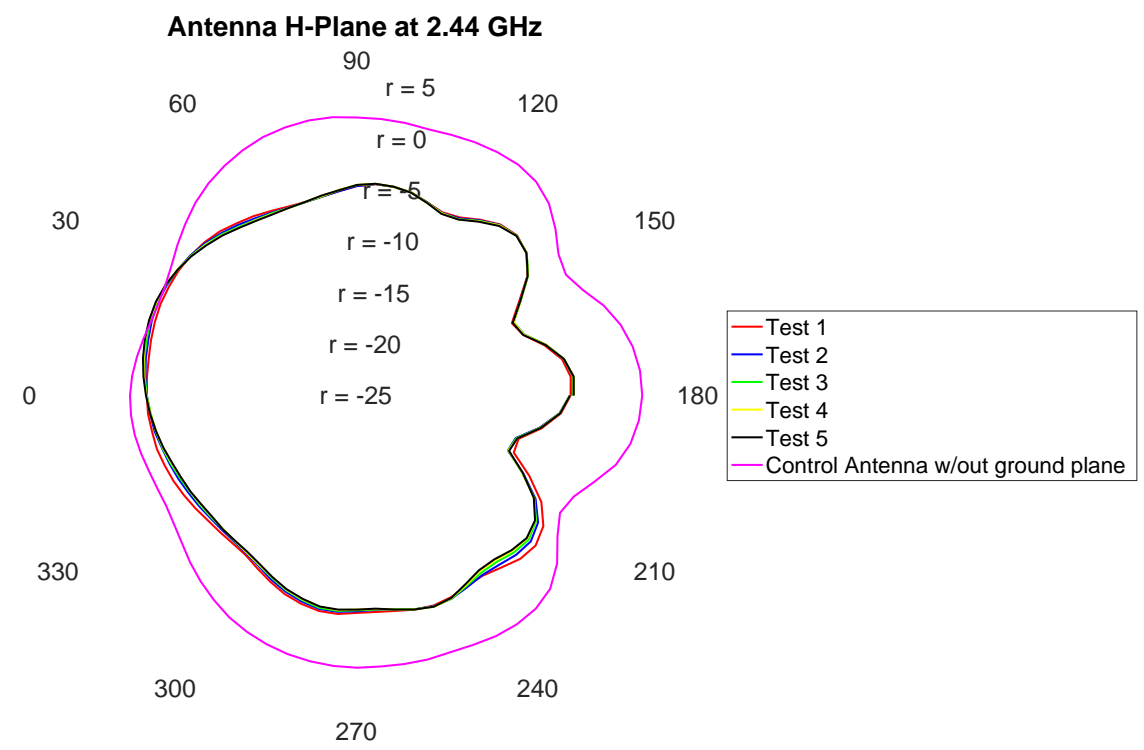

Figure 5.18: Comparison of Patch Antenna H-plane with and without a Copper Ground Plane.

The boresight gain of the patch antenna with the copper ground begins to decrease in strength at higher frequencies which is similar to the gain measured in the antennas 
datasheet [38]. The radiation pattern is still different than the datasheet as it still uses a substrate with a different permittivity than the data sheet. However, compared to the antenna measurements taken in this thesis without a ground plane, the antenna with the copper ground plane forms two new nodes in the E-plane. Additionally, the antenna has a reduction in the size of the back lobe of both the E-plane and H-plane as the ground plane appears to be reflecting more of the signal.

\subsection{Scanning Electron Microscope and FTIR Spectroscopy}

Since no degradation in performance was seen in the patch antennas, a scanning electron microscope and Fourier Transform Infrared Spectroscopy were used to try to determine whether the powdery substance on the surface of the patch antenna was a stable or unstable oxide. AO exposure is known to create oxide layers on the surface of the materials it interacts with [60]. The determination of a stable or unstable oxide on the surface would indicate if the erosion would continue at the same rate [60]. An unstable oxide causes the steady progression of erosion on the antenna surface, which would eventually expose the copper etching on the interior of the antenna. When pure copper is exposed to AO it can form cuprous oxide and cupric oxide, both of which have higher resistivity than pure copper and could change the antenna performance [61]. The resistivity of the copper etching directly effects the amount of power the antenna can transmit, as any power lost to resistance will be turned into heat due to the process of resistive heating [26]. Since the gain of an antenna is directly related to the amount of power the antenna can transmit, any losses in the antenna's power will result in a lower gain. The AO erosion on all the tested antennas did not expose the copper etching in the interior of the antenna and therefore no copper oxides were discovered. Using the SEM, a control, an unexposed, and a 48-hour 
exposed antenna were observed at various magnifications. Figure 5.19 and figure 5.20 show a comparison of the 48-hour antenna and the control antenna, respectively.
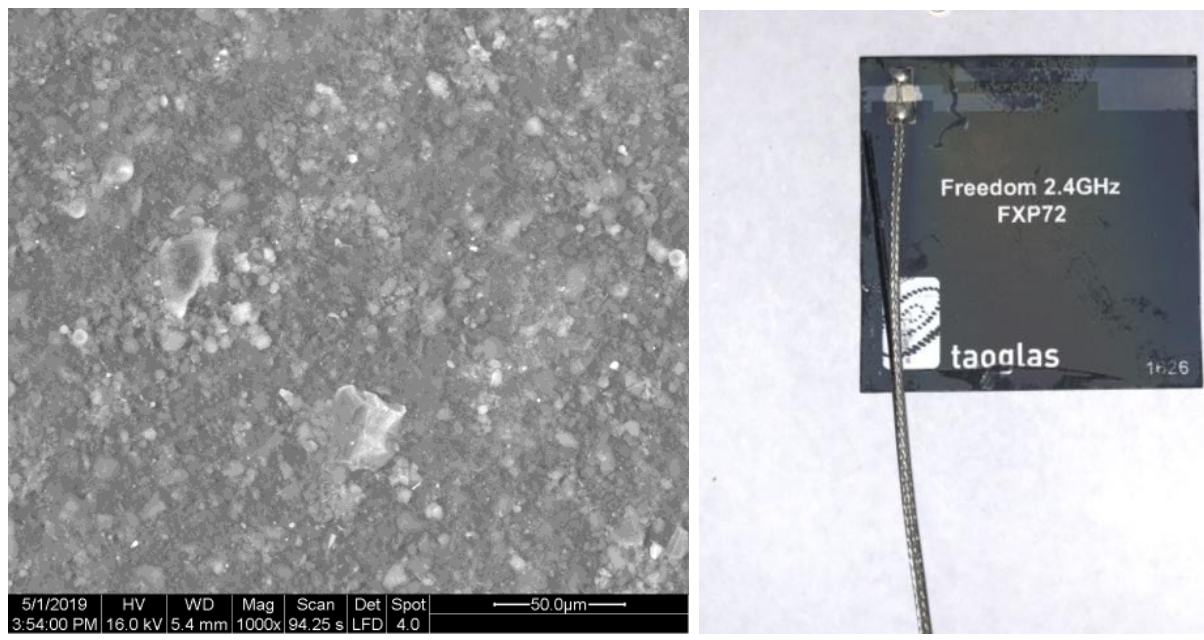

Figure 5.19: Patch Antenna Surface (Left) and Patch Antenna (Right) After 48 Hours of Exposure.
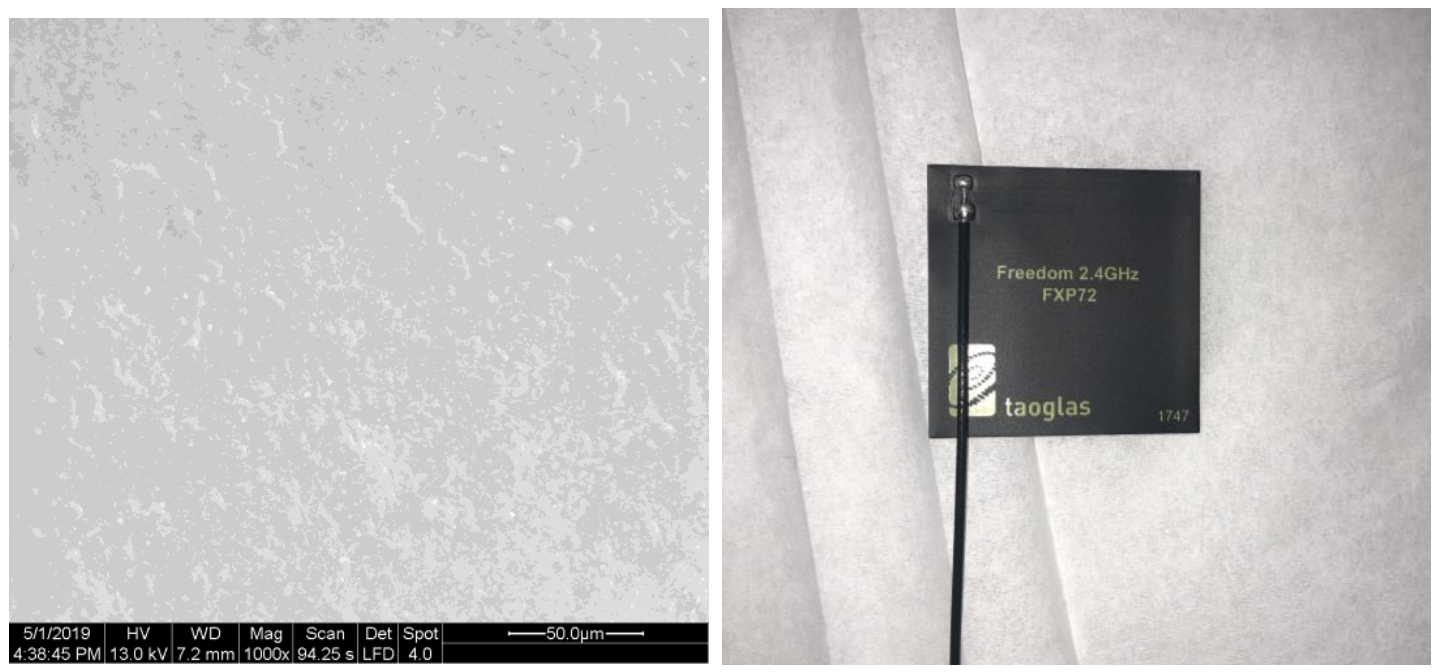

Figure 5.20: Control Patch Antenna Surface (Left) and Patch Antenna (Right).

The surface of the 48-hour patch antenna is significantly rougher than the surface of the control antenna. The change in the surface is due to the interaction of the AO with the surface material. The rougher surface along with the mass loss values seen in the samples 
also indicates that the molecular structure of the outer coating of the antenna is being eroded by the AO. During the imaging of these samples it was also discovered that the conductivity of the surface of the control and 48-hour sample were different. For the 48hour sample, a higher accelerating voltage was used, which allows for a higher signal to noise ratio for the image. The ability to utilize a higher accelerating voltage in the SEM means that the surface of the 48-hour sample was more conductive than the surface of the control sample. When the control sample was examined at the same accelerating voltage as the 48-hour sample, charging effects were noticed in the control images. Charging effects are due to the buildup of static electricity on the sample which can cause a grainy or scratched texture to appear on the images. Figure 5.21 shows an example of the charging effects seen on the control antenna in the red circle.

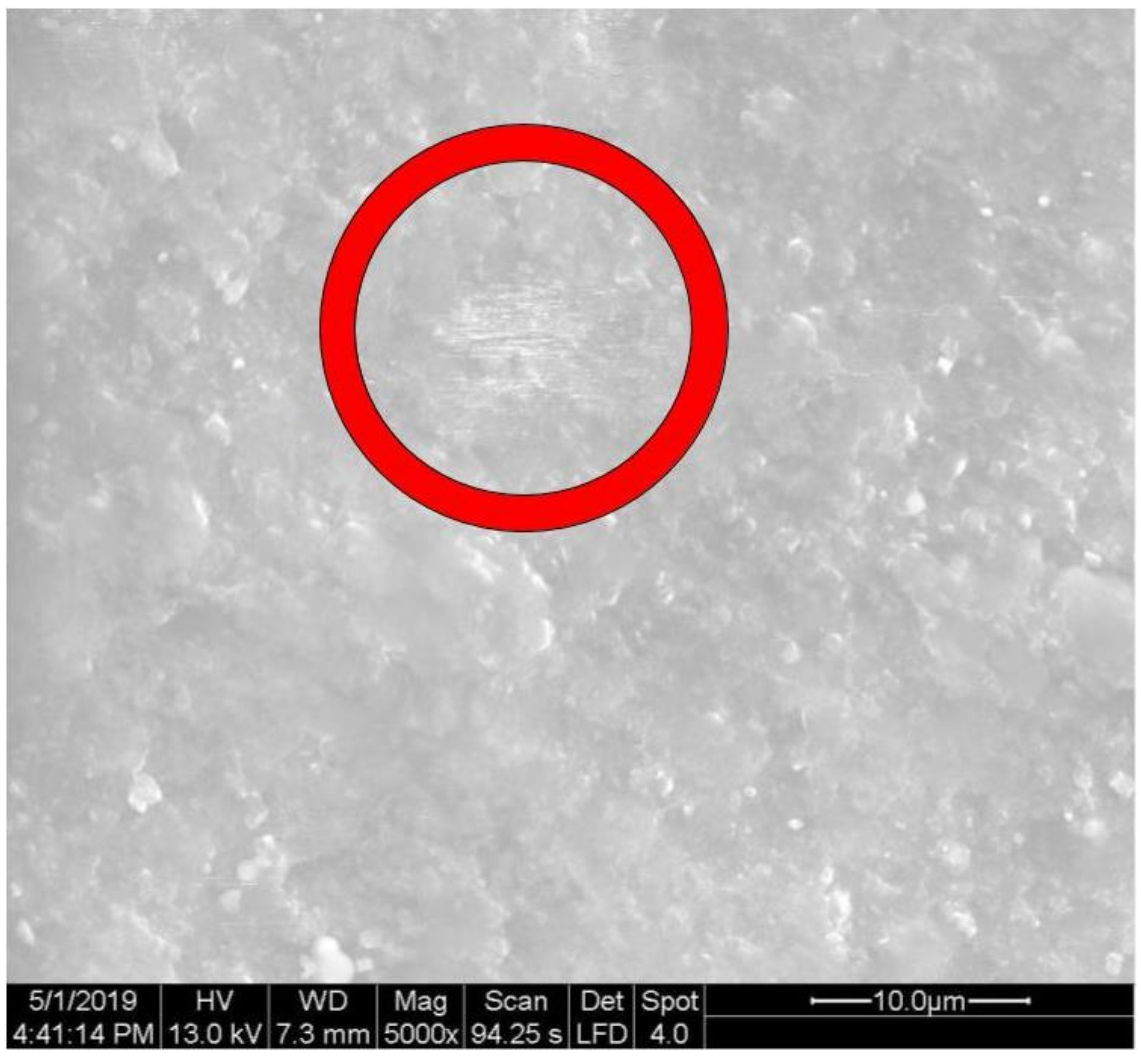

Figure 5.21: The Effects Charging Has on Images Taken by the SEM. 
To get a better understanding of the effects that the $\mathrm{AO}$ was having on the patch antenna surface, the Fourier Transform Infrared Spectrometer was used on a control sample, a 24hour exposed sample, and a 48-hour exposed sample. The chart shows the \% Transmittance as a function of the wavenumber ranging from $4000-400 \mathrm{~cm}-1$. The dips seen in the IR spectra are referred to as peaks and represent different chemical bonds that are either bending or stretching as a reaction to being bombarded by infrared radiation. The chemical bond is determined by looking at the wavenumber at the lowest point in each peak, the width of the peak, and the overall depth of the peak. A plot of the untested control sample can be seen in figure 5.22 .

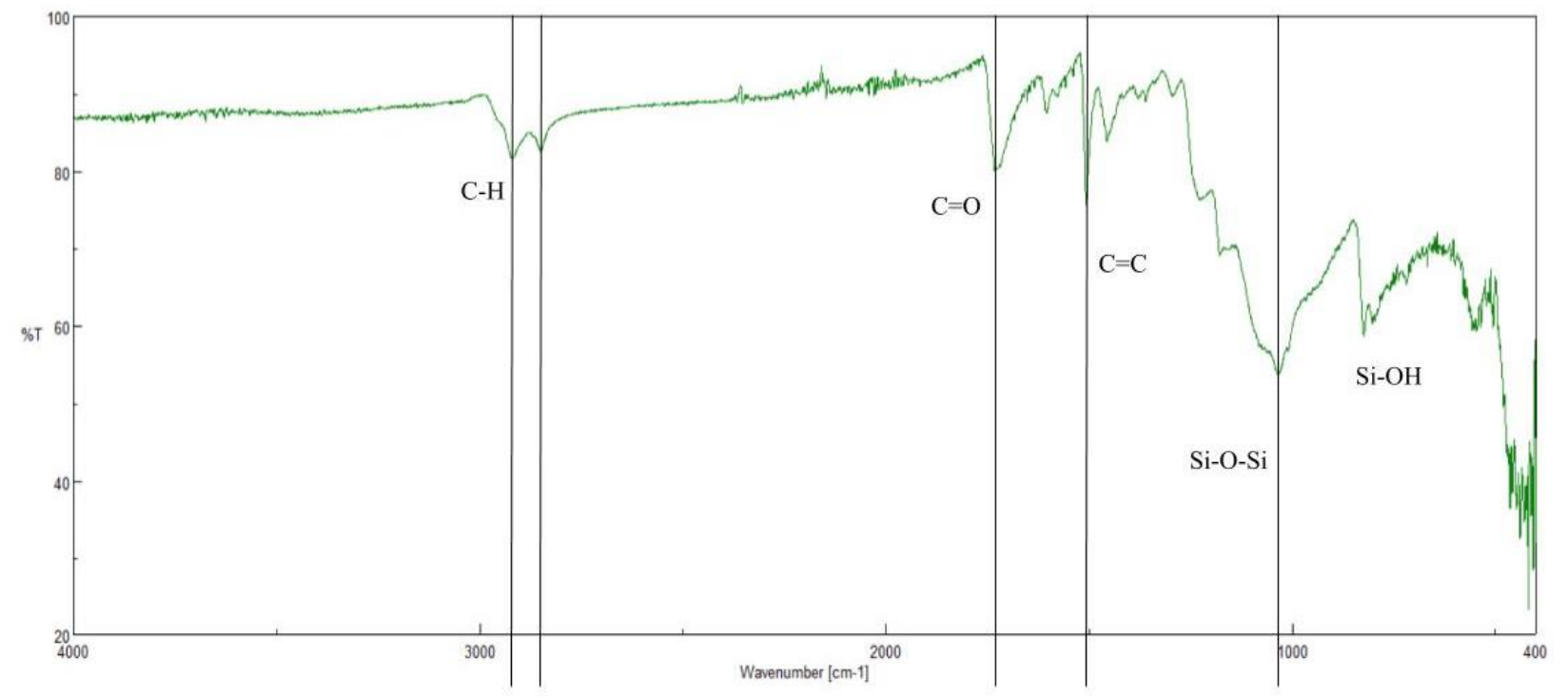

Figure 5.22: Attenuated Total Response IR Spectrum of Control Antenna.

The FTIR Spectrometer utilizes a software program called KnowItAll, that attempts to identify the material by analyzing the peaks in the data. Unfortunately, the program was unable to automatically identify the specific material that is on the surface of the patch antenna. To manually identify the most likely material that produced the graph in figure 
5.22, an FTIR Spectrometry Identification chart was used to identify the individual bonds for each peak [62]. A table of each peak's wavenumber and the corresponding chemical bond that occurs there can be seen in table 5.6.

Table 5.6: Peaks of IR Spectra and the Corresponding Chemical Bonds

\begin{tabular}{ccc}
\hline Wavenumber & Bond & Bond Movement \\
\hline 2918.25 & $\mathrm{C}-\mathrm{H}$ & Stretching \\
2849.79 & $\mathrm{C}-\mathrm{H}$ & Stretching \\
1726.46 & $\mathrm{C}=\mathrm{O}$ & Stretching \\
1504.69 & $\mathrm{C}-\mathrm{C}$ & Bending \\
1454.55 & $\mathrm{C}-\mathrm{C}$ & Bending \\
1227.95 & Si-O & Stretching \\
1175.4 & Si-O & Stretching \\
1036.07 & Si-O-Si & Stretching \\
822.973 & C-H & Bending \\
\hline
\end{tabular}

Based off the peaks seen in the chart and figure 5.22 of the untested sample, it is suspected that the surface material of the patch antenna is a polyester with a silica additive. Per the IR spectrum table provided by Sigma Aldrich, the peak at $1727 \mathrm{~cm}-1$ signifies a $\mathrm{C}=\mathrm{O}$ bond found in esters [62]. The specific polyester that is thought to make up the surface of the antenna is polybutylene terephthalate (PBT) due to the peaks seen at 2918, 2849, 1726,1504 , and $1454 \mathrm{~cm}-1$. The reason PBT is thought to be the polyester used rather than other types, is because the spectrum seen in figure 5.22 lacks a peak at the $1339 \mathrm{~cm}-1$ wavenumber, which is seen in the more common polyethylene terephthalate (PET) [63]. 
The broad peak from 1300-1000 cm-1 typically signifies a Si-O-Si bond, which is typically siloxane [4]. Siloxane is found in many types of silicone resins and silicone resins are sometimes used to strengthen polyesters and make them more flexible [64]. The specific silicone resin is not known. The doublet of peaks at the 2918 and $2849 \mathrm{~cm}-1$ wavenumbers have been seen in previous studies to signal C-H stretching of the benzene ring, but could also be part of the silicone resin as some polysiloxanes can have $\mathrm{C}-\mathrm{H}$ bonds [51]. In figure 5.23 , the control sample is shown with the red line, the 24-hour sample is represented by the green line, and the 48-hour sample is shown in blue.

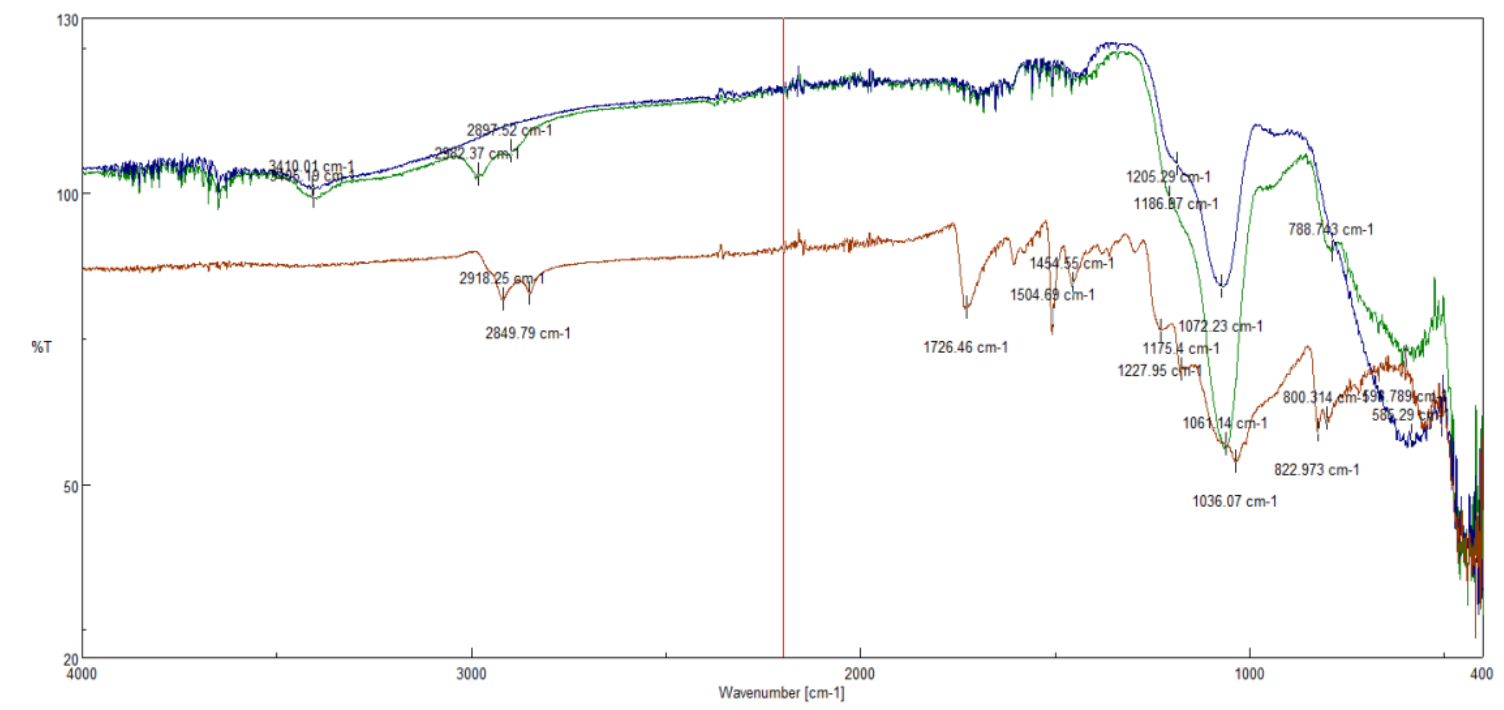

Figure 5.23: Attenuated Total Response IR Spectrum of the Control Antenna (Red), 24-Hour Sample (Green), and 48-Hour Sample (Blue).

From the chart, it can clearly be seen that after the 24 hours of AO exposure, the peaks in the $1800-1400 \mathrm{~cm}^{-1}$ and $1000-800 \mathrm{~cm}^{-1}$ range are gone. This means that the interaction with AO has broken these chemical bonds and removed the outer layer of ester, C-C, and $\mathrm{C}-\mathrm{H}$ bonds from the sample. Additionally, after 48 hours of $\mathrm{AO}$ exposure, the peaks in the $3000-2800 \mathrm{~cm}-1$ range have disappeared as well and a small weak peak at $3410 \mathrm{~cm}-1$ has 
formed. This suggests that the polyester that was present in the patch antenna before AO exposure has been removed from the outer surface. Underneath the new outer layer of material the ester is still present, but all the carbon bonds are no longer present on the surface. The new weak peak at $3400 \mathrm{~cm}-1$ represents an $\mathrm{OH}$ alcohol that formed after stripping away the polyester from the surface of the patch antenna while leaving behind the silica. Previous studies have shown that when AO interacts with silicone, a silicon dioxide glass forms on the surface [8]. This silicon dioxide is a stable oxide that is resistant to further AO erosion. By comparing the IR spectra seen in the 48-hour test, it can be seen that the peaks observed match those of silicon dioxide. For both of the exposed sample IR spectra the $\%$ Transmittance is above $100 \%$ due to the roughness of the reference sample compared to the surface of the measured sample. Figure 5.24 shows the IR spectra of silicon dioxide.

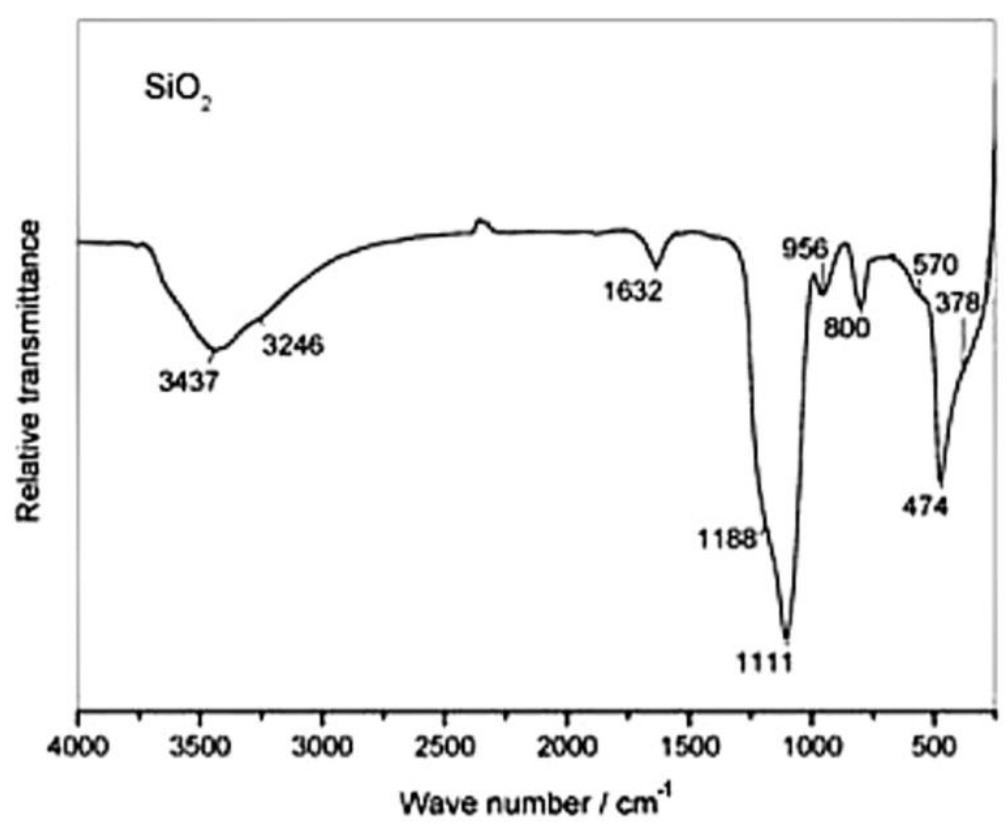

Figure 5.24: Silicon Dioxide IR Spectra [65]. 
These results are further supported by the visible color change seen on the surface of the patch antenna. When a thin layer of silicon dioxide forms, its color will change depending on the thickness of the layer. Figure 5.25 shows how the color of silicone dioxide changes as the material's thickness increases, and figure 5.26 shows the color gradient seen on the 48-hour patch antenna.

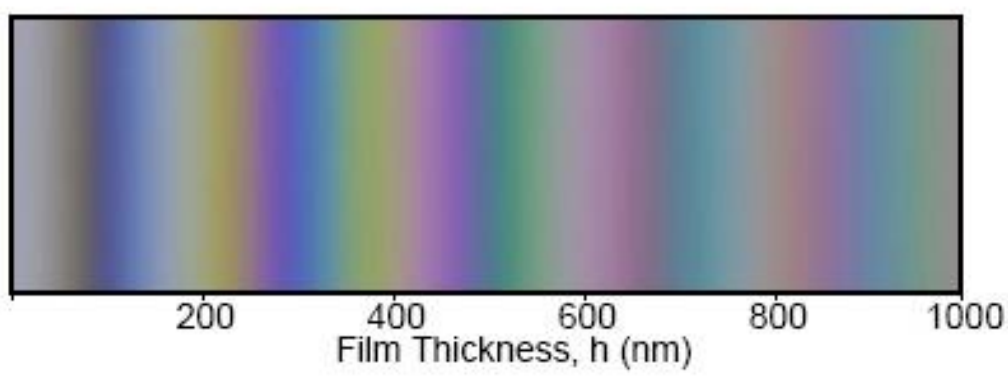

Figure 5.25: Silicon Dioxide Thickness vs Observed Color at $0^{\circ}$ Incidence Angle $[66]$.

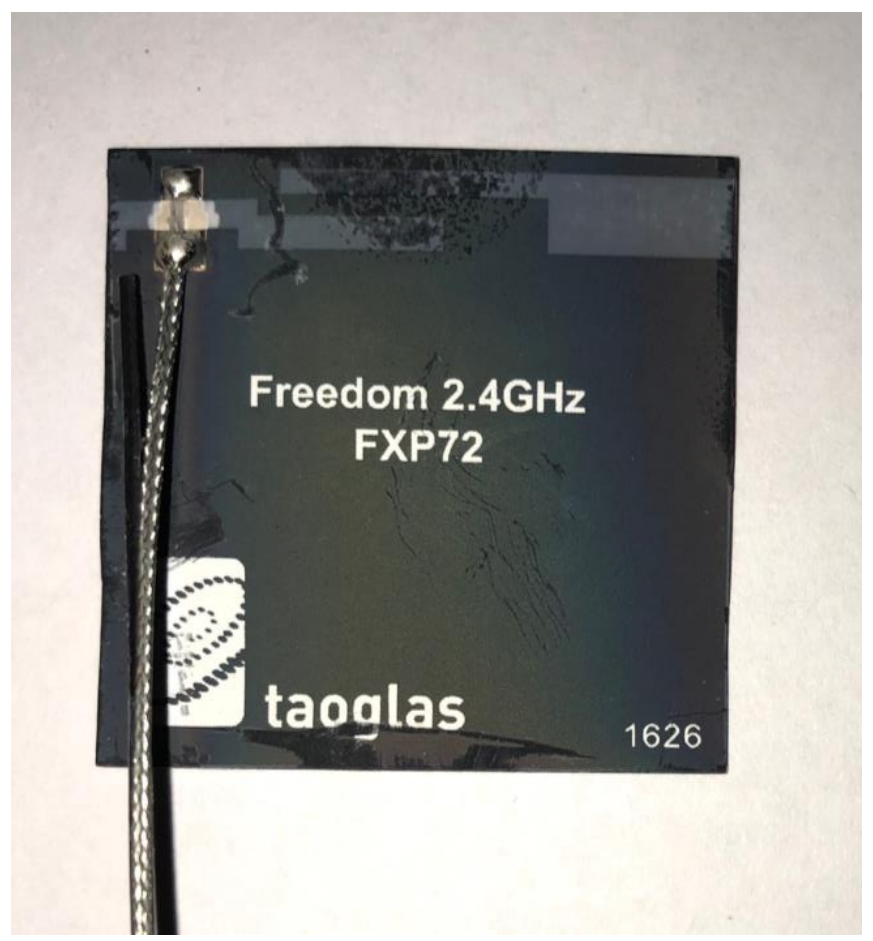

Figure 5.26: Color Gradient Seen on the 48-Hour Patch Antenna Sample. 
By comparing the chart in figure 5.25 to the 48-hour exposed patch antenna in figure 5.26 , it is possible to see the color on the antenna matches the colors of varying degrees of silicon dioxide thickness. At the center of the patch antenna the silicon dioxide layer appears to be between 200-250 $\mathrm{nm}$. Moving towards the edge of the antenna the silicon dioxide layer appears to thin as the color shifts from yellow to blue to grey. Based off this observation, it is thought that the antenna will form a stable oxide layer of silicon dioxide that will minimize AO erosion over longer exposure times. This was supported by the earlier observation that the mass loss seen in the 48-hour samples was not double the mass loss of the 24-hour samples. 


\section{Chapter 6}

\section{CONCLUSION}

The results from the testing of the Freedom $2.4 \mathrm{GHz}$ Patch Antenna show that exposure to $\mathrm{AO}$ erodes the exterior of the connecting wire and the surface of the patch antenna itself. Although there was mass loss seen in both the 24-hour and 48-hour exposures, neither exposures saw an apparent change in the boresight gain or radiation pattern of the patch antenna. From the witness samples in the tests, each test experienced between 3.2-6.4 months of on-orbit exposure for the ram direction of a spacecraft orbiting in a $28.5^{\circ}$ inclined, $400 \mathrm{~km}$ altitude, orbit. From the mass measurements collected for each tested antenna, it was apparent that the amount of AO seen by each of the antennas varied based on the antenna's placement in the chamber. For all tests, except test 3 , the samples in positions 2 and 5 saw the highest mass loss in their respective troughs. This is likely due to the samples placed in positions 2 and 5 being the closest to the RF electrode. This trend could also be observed by visual comparison of the antennas after exposure to AO. Even though the antennas showed visual changes and changes in mass, there was no change in performance. By comparing the connecting wire of the antenna in the 24-hour test, it could be seen that any part of the cable that was closer to the center of the chamber, and therefore closer to the RF electrode, would be clearly more eroded than the parts of the wire that were further from the center. The exposed antennas also had a powder-like substance on the surface after testing that was white and could easily be rubbed off the antenna. Prior to $\mathrm{AO}$ exposure it was not possible to rub off material from the surface of the patch antenna, meaning the powdery material on the surface of the exposed patch antennas possessed significantly weaker bonds to the underlying material. 
After comparing results from the performance of the antenna before and after AO exposure, it was clear that boresight gain in the frequency range from $2.39-2.49 \mathrm{GHz}$ was unaffected. The null hypothesis was that the average gain of the patch antenna before AO exposure would be equal to the average gain of the patch antenna after $\mathrm{AO}$ exposure. This was confirmed by using a paired samples t-test for the overall frequency $(2.39-2.49 \mathrm{GHz})$, the lower frequencies (2.39-2.4395 GHz), and the higher frequencies (2.44-2.49 GHz), all of which showed that it was not possible to reject the null hypothesis. Visual inspection of the radiation pattern in the E-plane and H-plane was also unable to show any notable difference in the patch antenna.

A scanning electron microscope and FTIR spectrometer were used to obtain a better understanding of the chemical process that was occurring on the surface of the patch antenna. From the images obtained using the scanning electron microscope, it could be seen that there was a change in the roughness of the patch antenna surface due to exposure to AO. Additionally, the SEM needed to be operated at different settings due to the fact that the conductivity of the surface of the antenna changes after being exposed to AO. From the data collected by the FTIR spectrometer a change could be seen in the molecular bonds on the surface of the patch antenna after being exposed to AO. The unexposed antenna had a spectrum that most resembled PBT with a silica additive as many of the key peaks seen in the untested sample matched the IR spectrum peaks of PBT. As the antenna was exposed to the corrosive properties of $\mathrm{AO}$, the $\mathrm{PBT}$ was eroded and removed from the antenna which was seen in the mass loss. The silica from the resin remained on the surface of the antenna and after interacting with the AO began to form silicon dioxide. This was seen in the IR spectra of the 24 and 48-hour antenna samples. The 24-hour sample still had some 
peaks that signified C-H stretching; however, the 48-hour sample did not contain any of those peaks. The 48-hour antenna showed an IR spectra that matched silicon dioxide and even possessed the $\mathrm{O}-\mathrm{H}$ alcohol that is seen in the $3400 \mathrm{~cm}-1$ region. The beard and broad peak seen in all three of the IR spectra around the 1300-1000 cm-1 range is an indicator of a Si-O-Si bond that is fairly distinct. An additional Si-O bond peak appeared at the 540 $\mathrm{cm}-1$ region that helps to confirm the silica additive. Visual inspection of the post exposure antennas also showed evidence of a silicon dioxide layer being left behind. When silicon dioxide is observed in thin layers ranging in the hundreds of nanometers, the apparent color of the silicon dioxide will change depending on the thickness of the material. A color gradient could be observed on the patch antenna that matched the colors seen in silicon dioxide of varying thickness. Based on the colors seen on the antennas, the thickest portion of the silicon dioxide is at the center of the antenna and grows thinner as it gets closer to the edges. It is possible that the gradient comes from the antenna being down into the trough. Therefore, the edges of the antenna that are closer to the wall of the trough are not experiencing the same AO flux as the part of the antenna in the center of the trough due to a lower view factor to the RF electrode.

Since silicon dioxide is forming on the surface of the antenna it is believed that further exposure to $\mathrm{AO}$ will increase the amount of silicon dioxide until the entire surface is coated. Silicon dioxide is naturally resistant to AO degradation; an outer layer would act as a naturally occurring shield for the antenna and minimize AO erosion, assuming the silicon dioxide surface does not crack. Previous studies have shown that silicon dioxide exposed to AO can crack and cause further erosion from the crack attenuation. Since no degradation in antenna gain or radiation pattern was observed after $\mathrm{AO}$ exposure, it appears that the 
silicon dioxide does not degrade the antenna gain if it is a thin layer. It is not clear how thick of a layer of silicon dioxide would be needed to fully minimize AO erosion for this antenna; however, if a thin enough layer can protect the antenna then it is unlikely that it will negatively affect the performance of the antenna. Based on this, it is recommended that any CubeSats that may have an antenna in the ram direction that will be exposed to AO for over 6 months in a $28.5^{\circ}, 400 \mathrm{~km}$ orbit, should also include a thin layer of silicon dioxide to the surface of the antenna or a silica additive to the surface material of the antenna. 


\section{Chapter 7}

\section{FUTURE WORK}

\subsection{Junior}

The outgassing chamber used for the experiments conducted in this thesis only has a couple of things that need to be improved. Currently there is no pressure gauge in the vacuum chamber. Calculations done by Charles Ward show that the setup will be able to pump down to pressures lower than the 200 mTorr required by ASTM E2089 however there is no pressure gauge that actively shows the chamber pressure. A pressure gauge added to the chamber would allow for the pressure to be continuously monitored for the entire 48 hours of outgassing. Throughout testing this year Junior had issues with leaking oil. The exhaust filter from the vacuum pump has had oil build up inside of it multiple times, which eventually leads to the oil leaking out and onto the ground. The cause of this leak should be further investigated and repaired to prevent the oil from leaking into the lab. It is unlikely that this caused issues with the outgassing in the chamber as the Kapton witness samples maintained the same percentage of mass loss during outgassing for each test.

\subsection{MAX}

Out of all the improvements that could be made to MAX the most useful one would be to characterize the AO flux at different locations in the chamber. In this thesis, it was observed that when using the trough, any samples closer to the center of the chamber saw higher mass loss and therefor a higher AO flux. A map of the AO flux seen at different locations in the chamber would help identify any hotspots in the chamber which would allow for future experiments to explain differences in their samples. Many tests would need 
to be conducted with witness samples over the entire sample plate to achieve meaningful results, however it was not conducted in this thesis as it was not within the scope of the thesis research. It would also be necessary to account for variations in the pressure of the chamber throughout the duration of the test. It is recommended that a mass flow controller be added to the manual air bleed valve. This controller could then be connected to the convectron gauge and, with a simple control loop, regulate the pressure in the chamber to a higher precision than what is currently possible.

Another useful addition to the AO chamber would be a new circular trough plate. This trough plate would consist of two semi-circle cutouts that would be equidistant from the air bleed valve. A semi-circle aluminum trough plate would allow for smaller samples, that are too thick to be placed in the thin sample plate, to be tested in the AO chamber. A trough plate with a circular cutout would allow for a much more even AO flux on the samples placed in it. For the antenna tested in this thesis it was clear that samples placed in the middle of the trough experienced a much higher AO flux so a circular trough cutout would allow for a more consistent erosion among the samples.

During testing, there were occasions when the convectron gauge in the chamber seemed to malfunction. This would only happen when the RF power was on and would usually only last for several seconds before returning to normal pressures. If a mass flow controller were to be added to the chamber, the convectron gauge would need to be fixed to ensure that the malfunction does not occur while the flow controller is active. If the gauge malfunctions, it would cause the controller to also malfunction and ruin any tests being conducted in the chamber. 
Another major improvement to MAX would be to fix the UV lamp to allow for synergistic testing of $\mathrm{AO}$ and $\mathrm{UV}$ radiation. The synergistic effects between $\mathrm{AO}$ and UV radiation would allow for more extensive testing to be conducted in future experiments. Students are currently working to get the UV lamp up and running and will hopefully have it finished in the next year.

\subsection{Anechoic Chamber}

The anechoic chamber is currently used by companies for antenna testing and has minimal improvements needed. The biggest improvement that the anechoic chamber could use would be a new vector network analyzer. An improved vector network analyzer would allow for wider frequency ranges to be tested and better vector error correction which would provide even more accurate measurements than the current vector network analyzer possesses. Another improvement would be a characterization of all the different errors in the system. A chart or guidebook of the various errors that would occur from different parts of the chamber setup would improve any tests conducted in the chamber by allowing the experimenter to quickly and easily account for error. It would also help identify any malfunctioning equipment during tests if the error is outside the expected bounds. The anechoic chamber could also use high frequency SMA cables to allow for testing of higher frequency devices in the chamber.

\subsection{Antenna Testing}

Test results from the tests conducted in this thesis raised new questions that need to be answered to better understand how patch antennas would perform when exposed to AO in orbit. The patch antennas in this test appeared to be forming a silicon dioxide layer on the surface after being exposed to AO. The next step in testing these antennas would be to 
compare the erosion rate over time to see if the silicon dioxide begins to protect the surface of the antenna from erosion. If the silicon dioxide does form a stable oxide and protective layer for the patch antenna it would be necessary to see how it affected antenna performance. This could be done by simply coating unexposed antennas in thin layers of silicon dioxide and testing them in an anechoic chamber or by running longer AO tests on antennas to achieve thicker layers of silicon dioxide.

Another test needed on the antenna would be to test the copper etching after it has been exposed to AO. When copper is exposed to $\mathrm{AO}$ it forms cuprous oxide and cuprite oxide which have different dielectric constants than pure copper. This could affect the signal that the antenna produces as the current traveling across the surface of the copper will being traveling through a more resistive material. To accomplish this test, it would be necessary to remove the outer protective coating from the patch antenna and expose the copper etching to AO. After the AO tests are completed the same process of performance testing could be used to observe any changes in the antenna parameters.

Finally, a test to measure how Kapton adding surface charge to the back of the antenna would affect the performance results of the antenna. Since Kapton is a dielectric material it could change the performance of the antenna during testing by acting as a thin layer of substrate. 


\section{REFERENCES}

[1] Pisacane, V. L. (2016). The space environment and its effects on space systems. Reston, VA: American Institute of Aeronautics and Astronautics.

[2] "Radiation: Satellites' Unseen Enemy” Available: http://www.esa.int/Our_Activities/Space_Engineering_Technology/Radiation_satellite s_unseen_enemy.

[3] Orbital debris: a technical assessment, Washington, D.C.: National Academy Press, 1995.

[4] Corbett, J., "Micrometeoroids and Orbital Debris (MMOD)," NASAAvailable: https://www.nasa.gov/centers/wstf/site_tour/remote_hypervelocity_test_laboratory/mi crometeoroid_and_orbital_debris.html.

[5] Villela, T., Costa, C. A., Brandão, A. M., Bueno, F. T., and Leonardi, R., "Towards the Thousandth CubeSat: A Statistical Overview," International Journal of Aerospace Engineering, vol. 2019, 2019, pp. 1-13.

[6] Kramer, H. J., "Dove-1 and Dove-2 Nanosatellites,” Dove - Satellite Missions eoPortal DirectoryAvailable: https://directory.eoportal.org/web/eoportal/satellitemissions/d/dove.

[7] Banks, B. A., de Groh, K. K., and Miller, S. K., "Low Earth Orbital Atomic Oxygen Interactions With Spacecraft Materials.”

[8] Roe, R. R., SPACECRAFT POLYMERS ATOMIC OXYGEN DURABILITY HANDBOOK, 2014.

[9] Reddy, M. R. (1995). Effect of low earth orbit atomic oxygen on spacecraft materials. Journal of Materials Science,30(2), 281-307. 
doi:10.1007/bf00354389

[10] Banks, B. A., Groh, K. K., \& Miller, S. K. (2004). Low Earth Orbital Atomic Oxygen Interactions with Spacecraft Materials. MRS Proceedings, 851 . doi:10.1557/proc-851-nn8.1

[11]_"Atomic oxygen erosion,” European Space AgencyAvailable: https://www.esa.int/spaceinimages/Images/2017/05/Atomic_oxygen_erosion.

[12] Banks, B. A., Groh, K. K. D., and Miller, S. K., "Low Earth Orbital Atomic Oxygen Interactions with Materials," MRS Proceedings, vol. 851, 2004.

[13] Leger, L. (1983). Oxygen atom reaction with Shuttle materials at orbital altitudes Data and experiment status. 21st Aerospace Sciences Meeting. doi:10.2514/6.1983-73

[14] Harbaugh, J., "Materials International Space Station Experiment-X (MISSEX),"NASAAvailable: https://www.nasa.gov/mission_pages/tdm/missex/index.html. [15] "About the Materials International Space Station Experiment Facility," aboutMISSEAvailable: https://alphaspace.com/about-misse-ff.html.

[16] "Northrop Grumman CRS-11 Payload Overview," ISS US National LaboratoryAvailable: https://www.issnationallab.org/press-releases/northropgrumman-crs-11-payload-overview/.

[17] NASA Available: https://misse2.larc.nasa.gov/.

[18] A. S. for Testing and Materials. ASTM E2089 standard practices for gound laboratory atomic oxygen interaction evaluation of materials for space applications, 2015. 
[19] Minton, T. K., "Protocol for Atomic Oxygen -Iqesting of Materials in Ground-Based Facilities," Sep. 1995.

[20] Puig-Suari, J., Turner, C., Ahlgren, W., \& Twiggs, R. (n.d.). Development of the standard CubeSat deployer and a CubeSat class PicoSatellite. 2001 IEEE Aerospace Conference Proceedings (Cat. No.01TH8542).

doi:10.1109/aero.2001.931726

[21] Lee, S., CubeSat Design Specification.

[22] Langer, M., and Bouwmeester, J., "Reliability of CubeSats - Statistical Data, Developers' Beliefs and the Way Forward."

[23] Gao, S., Clark, K., Unwin, M., Zackrisson, J., Shiroma, W. A., Akagi, J. M., . . Sweeting, M. N. (2009). Antennas for Modern Small Satellites. IEEE Antennas and Propagation Magazine,51(4), 40-56.

doi:10.1109/map.2009.5338683

[24] VOLAKIS, J. (2018). ANTENNA ENGINEERING HANDBOOK. S.1.: MCGRAWHILL EDUCATION.

[25] Orban, D., and Moernaut, G., "The Basics of Patch Antennas," Orban Microwave Available: http://orbanmicrowave.com/the-basics-of-patch-antennas/.

[26] Notes, E., “Antenna Feed Impedance,” Electronics NotesAvailable: https://www.electronics-notes.com/articles/antennas-propagation/antennatheory/feed-impedance-matching.php. 
[27] (n.d.). Retrieved from http://licensing.fcc.gov/cgi-

bin/ws.exe/prod/ib/forms/reports/swr031b.hts?q_set=V_SITE_ANTENNA_FREQ.fil

e_numberC/File

Number/=/SATLOA2016111500118\&prepare=\&column=V_SITE_ANTENNA_FR

EQ.file_numberC/File Number

[28] "S-Band Antenna - GRACE-FO,” NASAAvailable: https://gracefo.jpl.nasa.gov/sband-antenna/.

[29] (n.d.). Retrieved from http://www2.csr.utexas.edu/grace/spacecraft/

[30] GRACE: Gravity Recovery and Climate Experiment Available:

http://www2.csr.utexas.edu/grace/operations/switch_maneuver.html.

[31] Escher, A., and Escher, A., "Inside Planet Labs' new satellite manufacturing site TechCrunch," TechCrunchAvailable: https://techcrunch.com/2018/09/14/insideplanet-labs-new-satellite-manufacturing-site/.

[32] Flock 1 Constellation - Satellite Missions - eoPortal DirectoryAvailable: https://directory.eoportal.org/web/eoportal/satellite-missions/f/flock-1.

[33] “A Dove's Eye View - Features - Po Bronson,” Alta OnlineAvailable: https://altaonline.com/a-doves-eye-view/.

[34] Klofas, B., and Leveque, K., “A Survey of CubeSat Communication Systems: 20092012" Available:

https://www.klofas.com/papers/Klofas_Communications_Survey_2009-2012.pdf.

[35] Rios-Olmo, R., and Miller, M., "Planet's Open Water Imaging - Geo-Accuracy Assessment." 
[36] Zackrisson, J. (n.d.). Wide Coverage Antennas. Retrieved from https://digitalcommons.usu.edu/smallsat/2007/all2007/93/

[37] Dybdal, R. (n.d.). Communication Satellite Antenna Testing. Retrieved from https://ieeexplore.ieee.org/stamp/stamp.jsp?arnumber=7108395

[38] FXP72.07.0053A Taoglas | Mouser. (n.d.). Retrieved from https://www.mouser.com/ProductDetail/Taoglas/FXP72070053A?qs=WUa1z/NV9+1 Y87nTMXKMXg==\&gclid=Cj0KCQiA_s7fBRDrARIsAGEvF8Qe1FLngjILhMePU 9By_5lgpEv_GHR4KCtwPLGqAew8Ct5PUIVeDtkaAl6_EALw_wcB

[39] Ward, C., "MODIFICATION OF A GROUND BASED ATOMIC OXYGEN SIMULATION APPARATUS TO ACCOMMODATE THREE DIMENSIONAL SPECIMENS,” thesis, 2018.

[40] Glicklin, M. J. (n.d.). Development of a Ground Based Atomic Oxygen and Vacuum Ultraviolet Radiation Simulation Apparatus. doi:10.15368/theses.2012.116

[41] Banks, B. A., Groh, K. K. D., Rutledge, S. K., and Difilippo, F. J., "Prediction of InSpace Durability of Protected Polymers Based on Ground Laboratory Thermal Energy Atomic Oxygen," Protection of Materials and Structures from the Low Earth Orbit Space Environment Space Technology Proceedings, 1999, pp. 89-100.

[42] Minton, T. K. (1995). Protocol for Atomic Oxygen Testing of Materials in GroundBased Facilities. Retrieved from https://ntrs.nasa.gov/archive/nasa/casi.ntrs.nasa.gov/19970037682.pdf. [43] "Seren IPS Home," Seren IPS HomeAvailable: http://www.serenips.com/. 
[44] Doan, A., "Synergistic Effects of Atomic Oxygen and Ultraviolet Radiation Exposure on Various Spacecraft Materials," thesis.

[45] Dash, G. (1995). How RF Anechoic Chambers Work. Retrieved from http://www.glendash.com/Dash_of_EMC/Anechoic_Chambers/Anechoic_Chambers. pdf

[46] Bloom, D. C., "DESIGN AND VERIFICATION OF A LABVIEW AUTOMATED ANTENNA RADIATION PATTERN MEASUREMENT SYSTEM,” thesis, 2018. [47] “HP 8719C, 8720C, 8722A/C Network Analyzer Operating Manual ”Available: https://literature.cdn.keysight.com/litweb/pdf/08720-90135.pdf?id=621021.

[48] "Model ELAZ75 ELEVATION OVER AZIMUTH POSITIONER” Available: http://www.sunarrfmotion.com/pdf/ELAZ75 Positioner.pdf.

[49] "ImageJA," ImageJ.

[50] Woodford, C., "How do electron microscopes work?," Explain that StuffAvailable: https://www.explainthatstuff.com/electronmicroscopes.html.

[51] Khandare, P., "Qualitative analysis of aramide polymers by FT-IR spectroscopy," International Journal of Engineering Science Invention, vol. 3.

[52] “FT/IR-4000 Series | JASCO,” JASCO Inc.Available: https://jascoinc.com/products/spectroscopy/ftir-spectrometers/models/ftir-4000series/.

[53] "A Summary of Error Propagation" Available: http://ipl.physics.harvard.edu/wpuploads/2013/03/PS3_Error_Propagation_sp13.pdf. 
[54] Glicklin, M., Abercromby, K., Ward, C., Reid, B., and Griffith, C., “Atomic Oxygen and Vacuum Ultraviolet Radiation Simulation Chamber at California Polytechnic State University, San Luis Obispo.”

[55] “T Test (Student's T-Test): Definition and Examples” Available: https://www.statisticshowto.datasciencecentral.com/probability-and-statistics/t-test/.

[56] "Dielectric Constant," Dielectric Constant: Definition, Units, Formula, Plastic Values \&Material ListAvailable: https://omnexus.specialchem.com/polymerproperties/properties/dielectric-constant.

[57] Kumar, K. P., "The effect of dielectric permittivity on radiation characteristics of coaxially feed rectangular patch antenna: Design \& Analysis," International Journal of Advanced Research in Computer and Communication Engineering.

[58] Li, R., Dejean, G., Tentzeris, M., Papapolymerou, J., and Laskar, J., "Radiationpattern improvement of patch antennas on a large-size substrate using a compact softsurface structure and its realization on LTCC multilayer technology," IEEE Transactions on Antennas and Propagation, vol. 53, 2005, pp. 200-208.

[59] Shukla, S., Verma, R., and Gohir, G., "Investigation of the effect of Substrate material on the performance of Microstrip antenna," IEEE Xplore Full-Text PDF:Available: https://ieeexplore.ieee.org/stamp/stamp.jsp?arnumber=7359350.

[60] Harty, T., "EFFECTS OF ATOMIC OXYGEN ON THE VACUUM-INDUCED MASS LOSS PROPERTIES OF A VARIETY OF SPACECRAFT MATERIALS,” thesis.

[61] Gusakov, A. G., Voropayev, A. G., Zheludkevich, M. L., Vecher, A. A., and Raspopov, S. A., "Studies of the interaction of copper with atomic and molecular 
oxygen," Physical Chemistry Chemical Physics Available:

https://pubs.rsc.org/en/content/articlepdf/1999/cp/a905829j.

[62] "IR Spectrum Table \& Chart," SigmaAvailable:

https://www.sigmaaldrich.com/technical-documents/articles/biology/ir-spectrumtable.html.

[63] Piccinini, P., Senaldi, C., and Alberto Lopes, J. F., "Fibre Labelling Polytrimethylene terephthalate - PTT- DuPont."

[64] "SILICONE RESINS," whatwhenhow RSSAvailable: http://what-whenhow.com/materialsparts-and-finishes/silicone-resins/.

[65] Musić, "Precipitation of amorphous $\mathrm{SiO} 2$ particles and their properties," Brazilian Journal of Chemical Engineering Available: http://www.scielo.br/scielo.php?script=sci_arttext\&pid=S0104-66322011000100011.

[66] Henrie, J., Kellis, S., Schultz, S. M., and Hawkins, A., "Electronic color charts for dielectric films on silicon" Available: https://www.osapublishing.org/DirectPDFAccess/04D728B1-EC7D-23B8A889BE5153C8B0C1_79472/oe-12-71464.pdf?da=1\&id=79472\&seq=0\&mobile=no. 
APPENDICES

Appendix A

PATCH ANTENNA TEST PROCEDURES

\section{A.1 Gain Testing}

Equipment needed:

- MicroLab Model S638A S-Band Horn

- FXP72 Freedom 2.4GHz Series Ground Coupled Antenna

- HP 8720C Network Analyzer

- LabView Antenna Measurement System (AMS) Network Analyzer Application

- SC104V Positioner Controller

- ELAZ75 Positioner System

Procedure:

- Place the MicroLab model S638A horn antenna in the anechoic chamber transmit position. Rotate the antenna axially to $0^{\circ}$ (vertical polarization). Place the FXP72 Freedom $2.4 \mathrm{GHz}$ Series antenna in the receive position

- Initialize the HP 8720C VNA using the following procedure.

1. Turn power ON (may require 15 minute warm-up, followed by a power cycle)

2. Press MEAS and select S21

3. Press FORMAT and select Log Magnitude

4. Press START and set the start frequency to $2.20 \mathrm{GHz}^{*}$

5. Press STOP and set the stop frequency to $2.80 \mathrm{GHz}$

6. Press MENU and set NUMBER OF POINTS to 401

7. Set POWER to $+10 \mathrm{dBm}$ 
- Carefully align the receive patch antenna to its beam peak by rotating SC104V channel 1 (polarization) and channel 2 (azimuth) until the received signal level on the VNA reaches a maximum. Calibrate the VNA using the following procedure.

1. Press the CAL hard-key and select CALIBRATE MENU

2. Select RESPONSE, then press the THRU key

3. When the thru path measurement is complete, press DONE RESPONSE

- Launch the VNA data storage LabView application: C:IAMS.V2lNewAMS: doubleclick on AMS2.1lb. Right-click inside the window and select View/Details. Double-click on HP8720 Network Analyzer Single Run.vi. This application stores data acquired from the VNA in a text file for processing and plotting. Change LabView parameters to match VNA settings: "Start/Stop Frequencies" and "Num of Points." LabView troubleshooting: see SparameterMeasViaLabView.pdf Measure input impedance for the patch antenna. Calibrate for "S22 1-Port" measurements: CAL, Calibrate Menu, S22 1-Port. Follow VNA instructions on connecting and measuring cal standards at the cable's RX antenna location. Shift measurement plane back to cal standard plane: scale/ref, electrical delay, $0.0635 \mathrm{~ns}$. Verify the calibration by reattaching cal standards and verifying correct readings. Specify "Ref1: Reverse S22 (B/R)" for RX antenna S11 measurements. The LabView application appends data to a user-defined filename in C:IProgram Files National InstrumentsLabVIEW 7.0lLabView Data. Capture VNA data by selecting RUN in the Labview application. Refer to the Lab Notebook for any problems. The data file contains two columns of 401 data points; the frequency $(\mathrm{Hz})$ at which each measurement was taken and measurement values in $\mathrm{dB}$. These 
values indicate the Antenna Under Test (AUT, the FXP72 Freedom 2.4GHz Series in this case) gain relative to the Narda standard gain horn.

\section{A.2 Radiation Pattern Testing}

For a linearly polarized RX AUT rotated azimuthally, the TX antenna orientation specifies AUT E-plane or H-plane pattern scans; horizontal (H) and vertical (V) TX polarizations, respectively.

Equipment:

- MicroLab S-Band Horn Antenna

- Cushcraft PC2415N Yagi-Uda Antenna

- FXP72 Freedom 2.4GHz Series Ground Coupled Antenna

- HP 8720C Vector Network Analyzer

- AMS Positioning System

- Labview AMS Single Frequency Stop-and-Go Application

- Labview AMS Advanced Controls Application

Procedure:

- Place the MicroLab Horn Antenna in the transmit position of the anechoic chamber with vertical polarization.

- Orient the coordinates marked on the antenna to match those shown in Figure A.1

- Initialize the HP 8720C VNA using the following procedure.

1. Turn power ON

2. Press MEAS hard-key and select S21

3. Press START hard-key and set start frequency to $2.4 \mathrm{GHz}$ 
4. Pres STOP hard-key and set stop frequency to $2.4 \mathrm{GHz}$

5. Press MENU hard-key and set NUMBER OF POINTS to 401

6. Set POWER to $+10 \mathrm{dBm}$

7. Press AVG hard-key and set IF Bandwidth to $100 \mathrm{~Hz}$

- Using the positioner system, carefully rotate the receive antenna until the $|\mathrm{S} 21|$ signal level shown on the VNA reaches a maximum value. Calibrate the VNA using the following procedure.

1. Press CAL hard-key and select CALIBRATE MENU

2. Select RESPONSE, then press the THRU key

3. When thru path measurement is complete, press DONE RESPONSE

All subsequent measurements are relative to this calibration
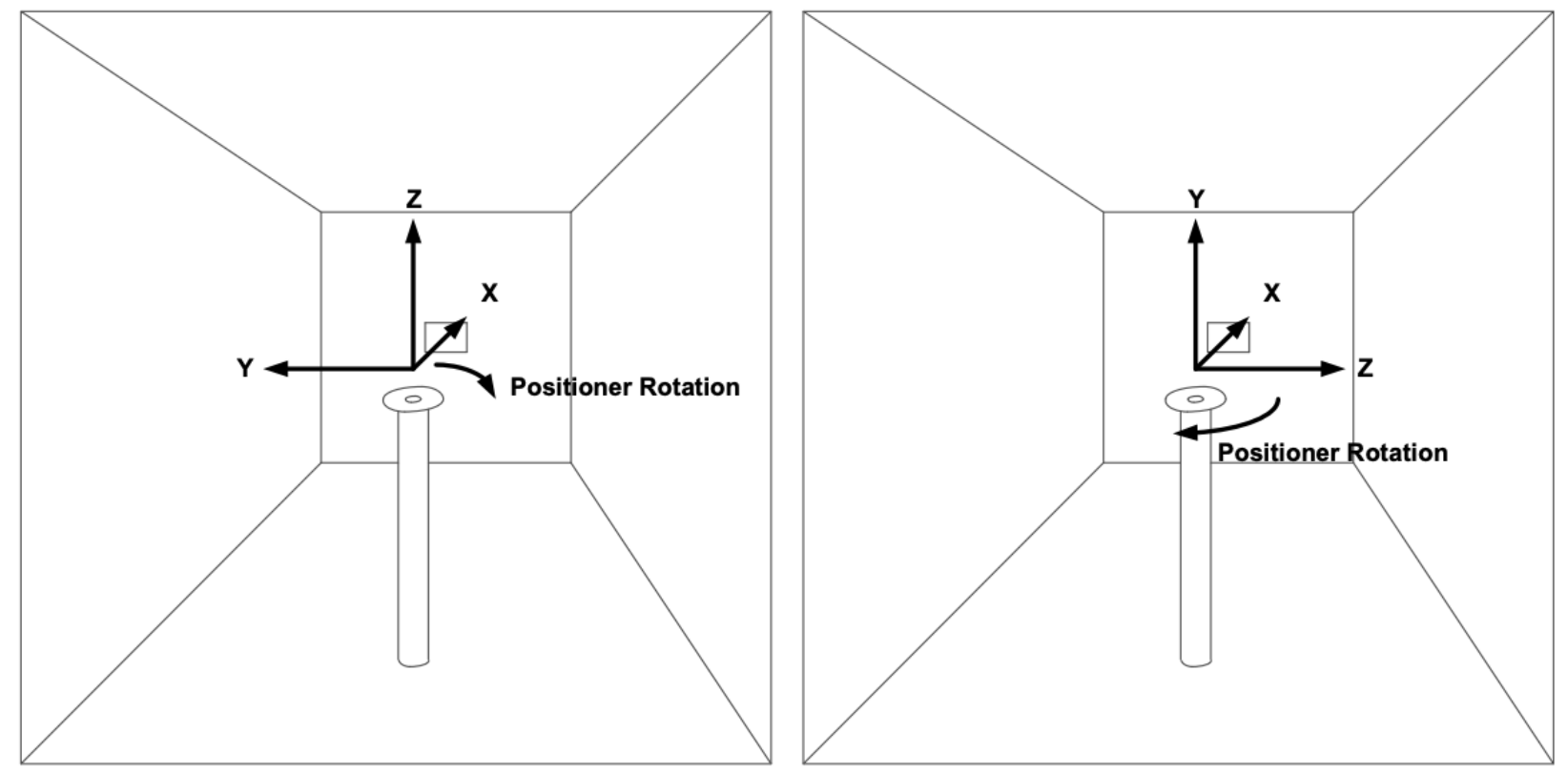

Figure A.1: Anechoic Chamber with Reference Frame Centered at the Test Antenna Position. 
- Launch the AMS Single Frequency Stop-and-Go application. Set the frequency to $2.4 \mathrm{GHz}$, start position to $0^{\circ}$, stop position to $360^{\circ}$, and increment to $5^{\circ}$. Open the "Labview Data" folder on the desktop and delete or rename any file named 'spsheet1.' Press Run button in the AMS application. The AMS will acquire VNA measurements while rotating the positioner clockwise in $5^{\circ}$ increments. The full $360^{\circ}$ measurement requires approximately 10 minutes to complete; the data is saved in 'spsheet1' within the 4 "LabViewData" folder. Rename this file to avoid appending additional data at a later time. The data file contains three data columns: roll and azimuthal angles in degrees, and $|\mathrm{S} 21|$ measurements in $\mathrm{dB}$. Use controller front panel to unwind cable: Enter 0, Start.

- Remove the U-Bolts from the Freedom Patch antenna's mounting plate. Polarization rotate the antenna $90^{\circ}$ to align the labeled coordinates to those of Figure 3.3 (H-polarization). Reattach the U-Bolts to the mounting plate and place the antenna on the positioner in the anechoic chamber. Rotate the source horn $90^{\circ}$ to polarization align to the Freedom Patch antenna and repeat the procedure above. 


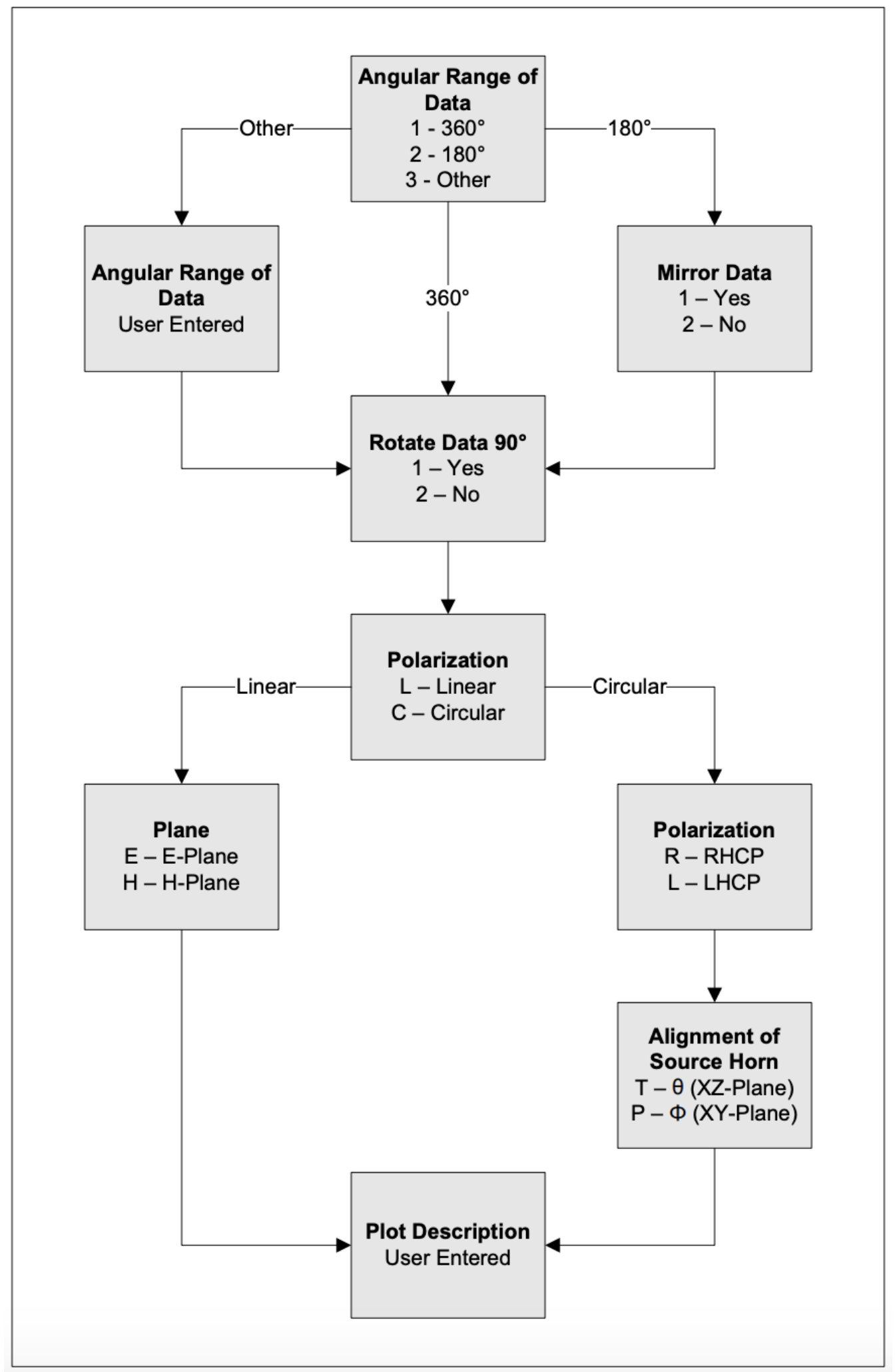

Figure A.2: Flow Chart for Choosing Radiation Pattern Measurement Settings. 
Appendix B

\section{AO TEST PROCEDURES}

The assembly instructions for MAX can be found in Charles Ward's thesis [22].

\section{B.1 Vacuum Operating Procedures}

1. Ensure that all vacuum control panel toggles are switched to the off position.

2. Make sure all service panels are closed and secure.

3. Flip the $1203 \Phi$ VAC breaker to the "on" position.

4. Open the ball valve to the pressurized air line.

5. Check the pressurized air regulator and ensure that it reads between 70-75 psi.

6. Turn on the "Main Power" on the vacuum control panel.

7. Turn on the Granville-Phillips 375 Vacuum Gauge Controller. Convectron gauge 3 (CG3) indicates chamber pressure in Torr.

8. Make sure all ports are closed, including the green nupro valve on the gas insertion line.

9. Turn on the Adixen ACP 28 Pump on the vacuum control panel.

10. Turn on the Chamber Roughing on the vacuum control panel. Monitor the chamber pressure on the Granville-Phillips 375 Vacuum Gauge Controller.

11. To shutdown, close the Chamber Roughing valve and Adixen ACP 28 Pump. Engage the vent valve, raise the hoist when pressure has equalized.

\section{B.2 MAX Operating Procedures}

Startup procedures:

1. Pump down the chamber as described in Appendix A.

2. Open the black nupro valve on the gas insertion line. Use the needle valve to adjust the pressure in the chamber to $175+/-10$ mTorr. 
3. Turn on the R301 generator.

4. Set the power to 125 Watts.

5. Turn on the MC2 controller.

6. Adjust the load and tune capacitors to $50 \%$. Make sure the operational mode is in Automatic for both load and tune.

7. Turn on the RF power using the switch on the R301.

The capacitors on the MC2 should auto adjust and find a stable operational point where the reflected power is 0 watts. If there is still reflected power or if the capacitors motors begin to oscillate, turn off the system and refer to the MC2 manual.

8. Once a stable point has been achieve, adjust the phase and magnitude potentiometers on the left hand side of the AT3 until the output on the MC2 controller is $0+/-25 \mathrm{mV}$.

9. Maintain a pressure between 165-185 mTorr and record temperature values every hour.

Shut down procedures:

1. Turn of the RF power switch on the R301 generator.

2. Close the black nupro valve on the gas insertion line.

3. Turn off the R301 and MC2 controllers.

4. Disconnect the Type N connector on the AT3 matchbox.

5. Vent the chamber in accordance to Appendix B.

6. Remove the sample containment cover plate, and weigh the samples in accordance with ASTM E2089: within 5 minutes. Caution: the DSS will be hotter than the ground plate, avoid contact with the DSS. 
7. Disassemble and store the apparatus as necessary. 
Appendix C

ADDITIONAL FIGURES

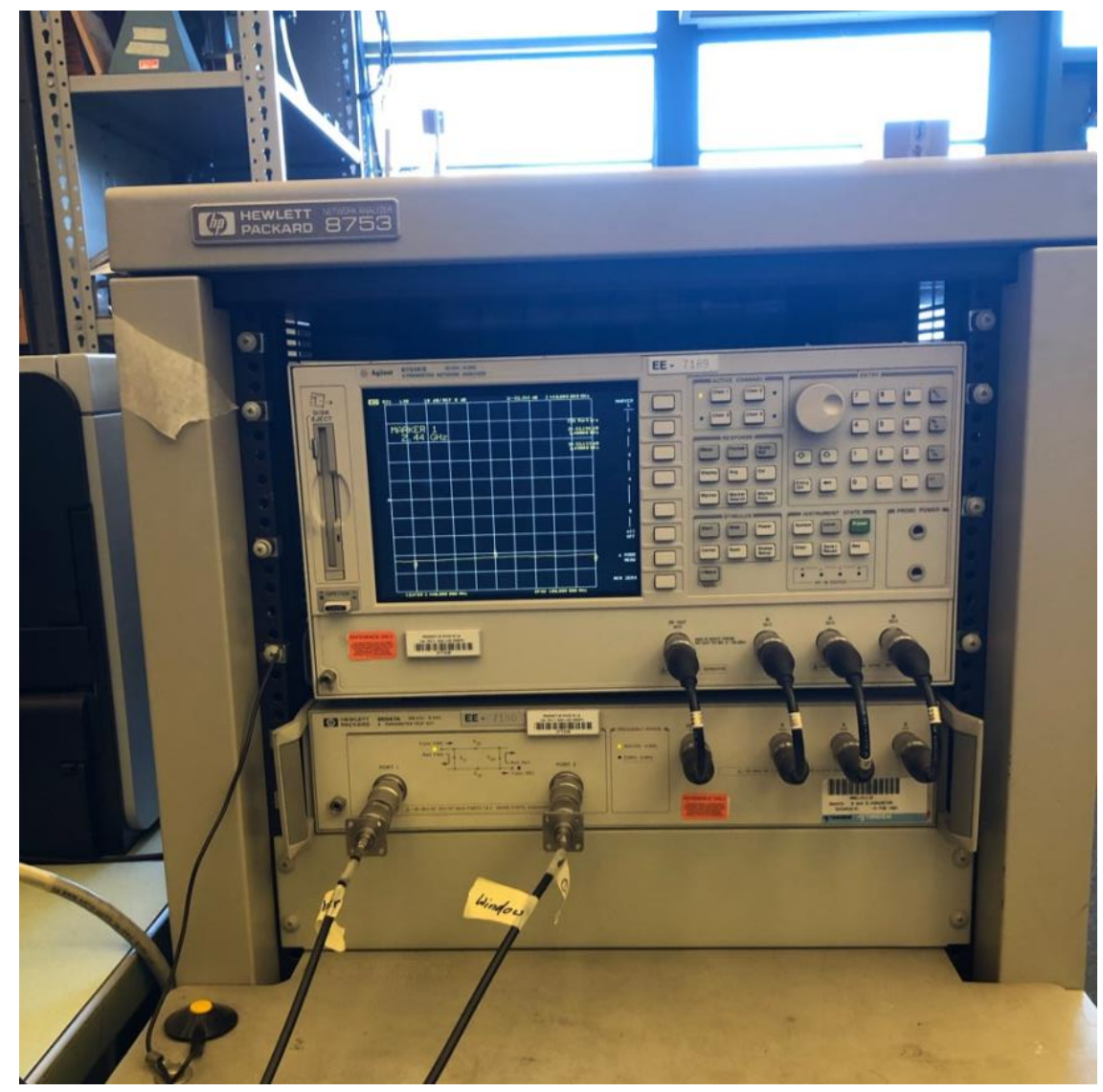

Figure C.1: VNA Used in the Anechoic Chamber. 


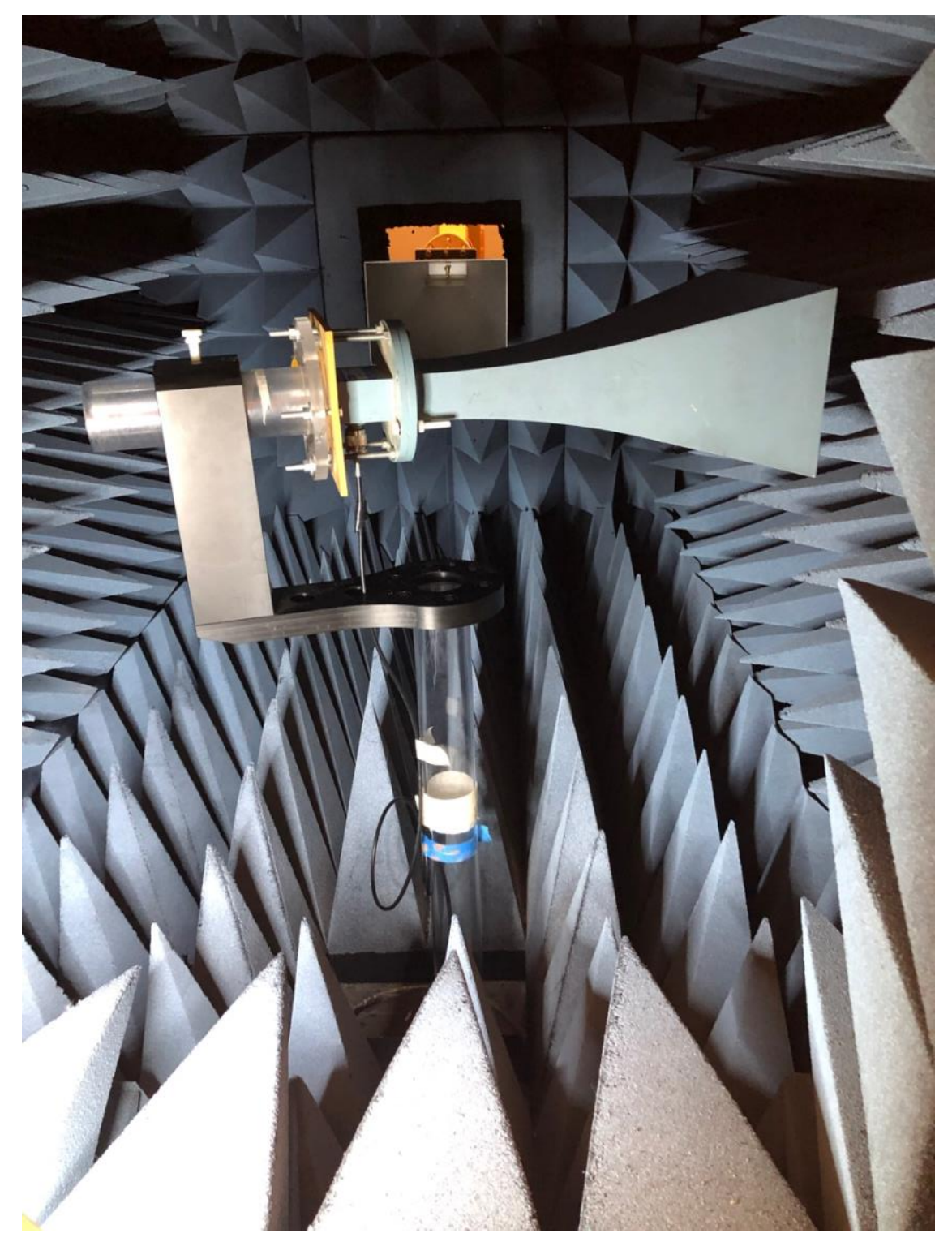

Figure C.2: Reference Gain Horn Antenna Used. 


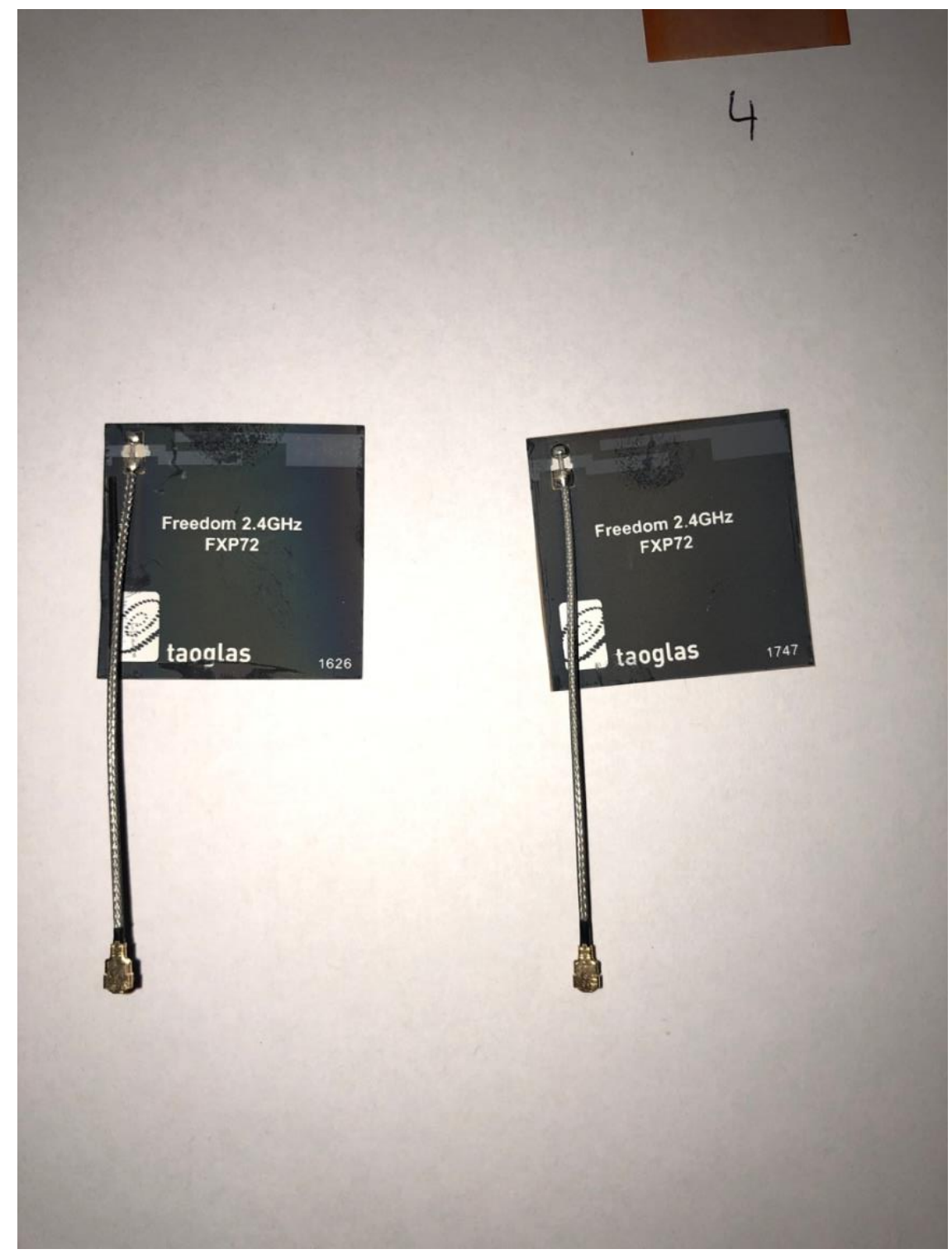

Figure C.3: 48-Hour Sample (Left) Compared to a 24-Hour Sample (Right). 


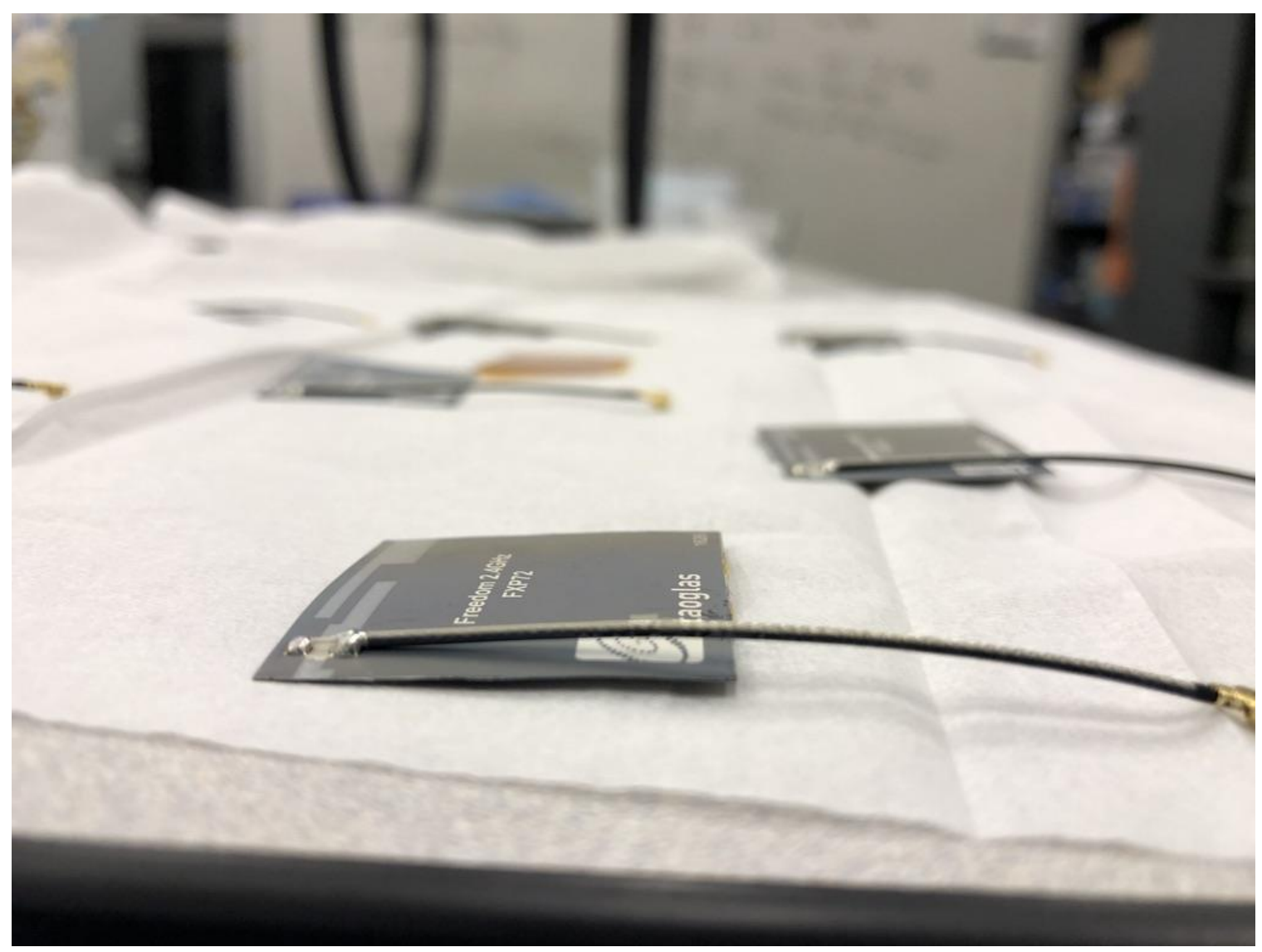

Figure C.4: Side View of 48-Hour Sample 


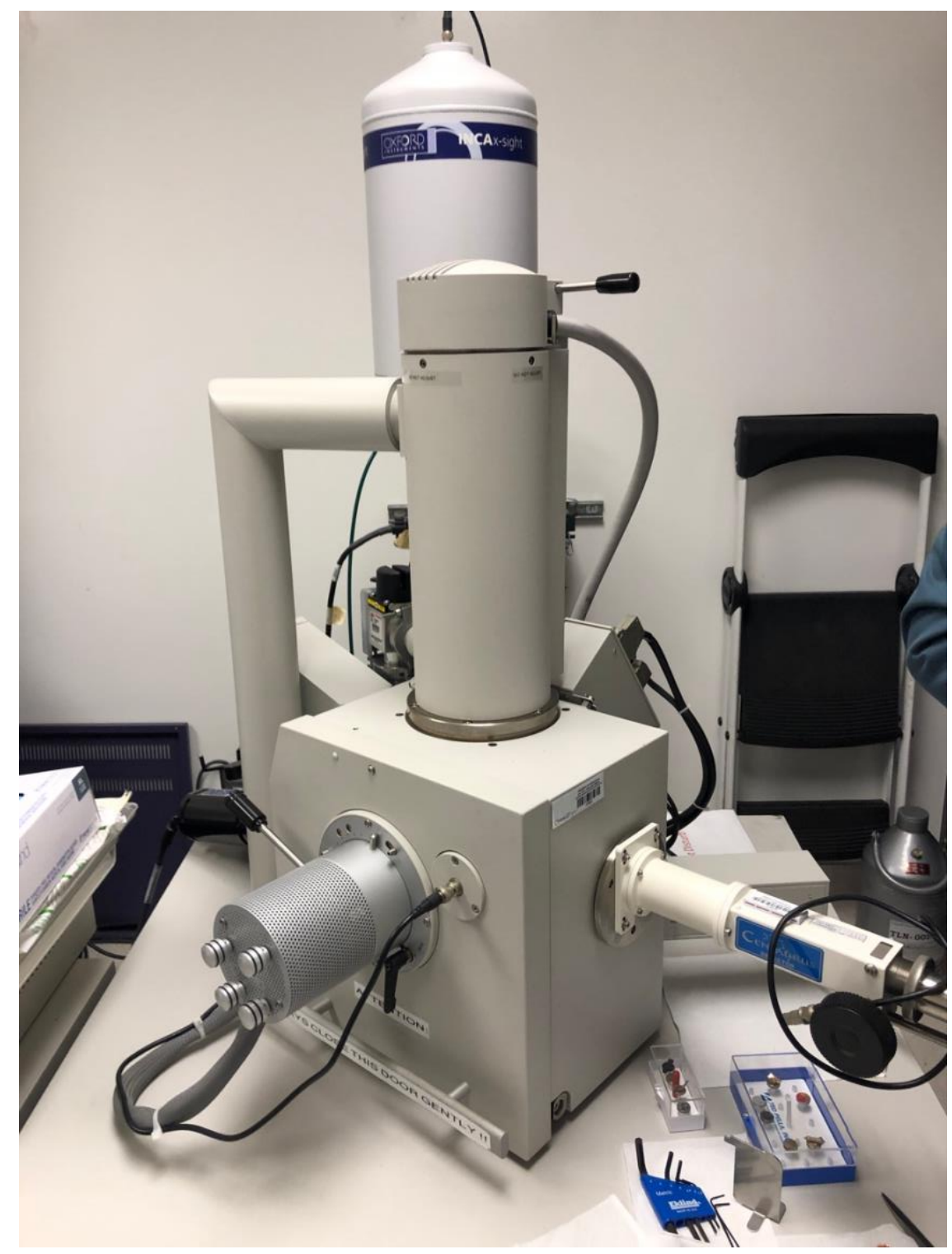

Figure C.5: Scanning Electron Microscope Used for Imaging Samples. 


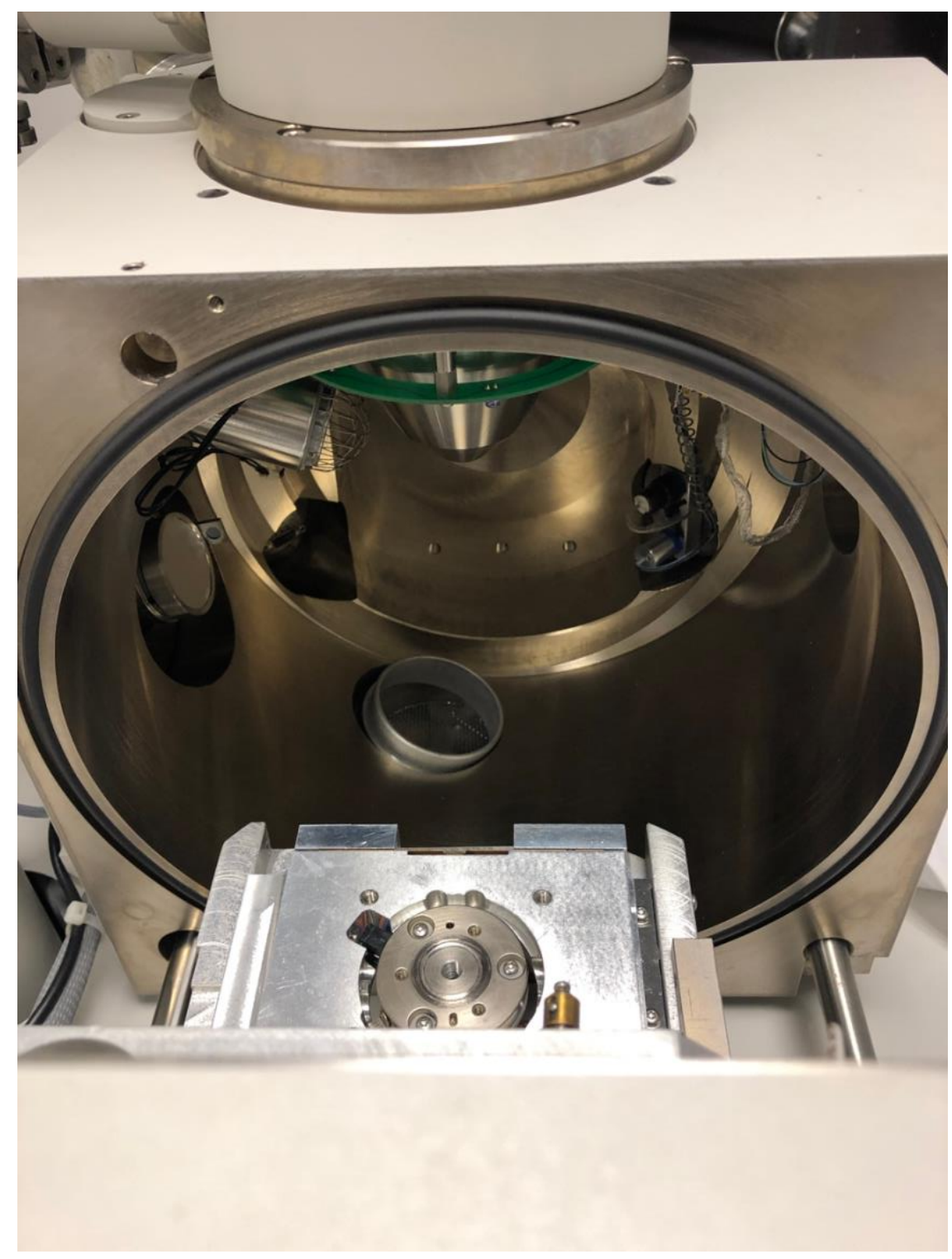

Figure C.6: Sample Tray and Interior of SEM 


\section{Appendix D}

\section{LESSONS LEARNED}

During the course of this thesis the testing done provided many lessons about conducting tests reliably and consistently especially when it comes to testing antennas. In this section some of the more important lessons that were learned will hopefully be passed on to anyone else needing to test antennas in future research.

Although the testing done in the anechoic chamber here on campus was much faster than is typical for antenna testing it still took much longer than was initially planned for. This is because there are many little details of each test that need to remain consistent for results to be valid. During testing the cable that was used to connect the AUT to the VNA was found to be creating huge amounts of loss in the system during one of the test. It is suspected that this was caused by the wiring inside of the cable fraying as the cable was relatively old. It was not possible to determine when exactly the cable began to cause the irregular losses that were measured so all of the data that was collected using that cable was no longer valid to use to compare antenna performance. In the future any cables used during the course of testing should be double checked every time they are used to ensure they are not tangled, twisted or snagged on a piece of equipment.

Another piece of equipment that should be constantly checked for degradations in performance are the adapters of used during testing. In this experiment a U.FL to SMA adapter was used to connect the antenna to the SMA cable. This adapter was a plug adapter that was press fit onto each antenna for testing and then removed after testing was completed. If the adapter was in anyway crooked when it was attached to the antenna the results measured by the VNA would be inaccurate. This required the adapter to be secured 
during radiation pattern testing to prevent the rotation of the positioner from causing a torque on the adapter. It is highly recommended that for future tests snap on adapters should be avoided if possible when selecting an antenna.

The VNA used during testing also had a few quirks that needed to be worked out. The biggest of these was that the VNA would occasionally have errors in its display when starting up. This would require the VNA to have its power cycled several times before it would begin to work properly. The process could take as long as 30 minutes to work out.

During the actual testing of the patch antenna it was necessary to ensure the antenna was always positioned in the exact same spot within the chamber. Since the anechoic chamber is open to other students this meant that the positioner and the mount needed to be consistently placed in the same location within the chamber. Additionally, the transmitting antenna need to also be in the exact same location and the exact same orientation. An easy way to ensure this happens is to use little bits of tape to mark the position or even a pen or pencil. That way even if another user changed the setup of the system it was easy to recreate the conditions used for testing.

With regards to the $\mathrm{AO}$ chamber it is imperative that the vacuum grease be properly applied during for every test. This was not done for later tests and as a result the amount of time it would take for the chamber to pump down to the pressure levels required increased significantly. When using the trough plate it was noticed that the aluminum tape used as a surface for the samples to rest on could be easily shaped to expose the underside of a sample. If this were to happen during a test the sample would experience erosion on the bottom and could lead to erroneous data. It is recommended that future tests use a stiffer surface that is easily leveled. 
Finally, when conducting any type of tests in general there is no such thing as too much data. Any opportunity to collect additional data should always be taken. When conducting analysis of the data there was never a time where too much data had been taken only times when it was thought that more data could be useful. 